Supporting Information (SI)

\title{
Optical control of CRAC channels using photoswitchable azopyrazoles
}

\author{
Xingye Yang, ${ }^{\dagger, \#}$ Guolin Ma, ${ }^{\ddagger}, \#$ Sisi Zheng, ${ }^{\S, \#}$ Xiaojun Qin, ${ }^{\dagger}$ Xiang Li, ${ }^{\dagger}$ Lupei Du, ${ }^{\dagger}$ Youjun \\ Wang, ${ }^{*} \S$ Yubin Zhou, ${ }^{*}$, Minyong $\mathrm{Li}^{*}, \uparrow, l$ \\ 'Department of Medicinal Chemistry, Key Laboratory of Chemical Biology, School of Pharmacy, Cheeloo \\ College of Medicine, Shandong University, Jinan, Shandong, 250012, China. \\ ${ }^{\ddagger}$ Center for Translational Cancer Research, Institute of Biosciences and Technology, College of Medicine, \\ Texas A\&M University, Houston, TX 77030, USA.

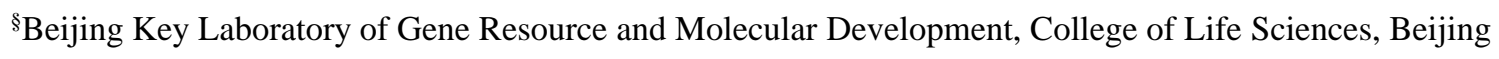 \\ Normal University, Beijing 100875, China. \\ "Helmholtz International Lab, State Key Laboratory of Microbial Technology, Cheeloo College of \\ Medicine, Shandong University, Jinan, Shandong, 250100, China. \\ \#These authors contributed equally to the work; *Manuscript correspondence to yubinzhou@tamu.edu, \\ wyoujun@bnu.edu.cn or mli@sdu.edu.cn.
}


Table of Contents

Design of photoswitchable CRAC channel inhibitors (piCRACs) (Figure S1)

Photochemical characterization of piCRACs compounds (Figure S2)

Light-inducible suppression on SOCE and NFAT1 nuclear translocation (Figure S3).....

UV Light-inducible suppression on SOCE (Figure S4). S5

Photochemical characterization of piCRAC-1 by HPLC and NMR (Figure S5) S6

Thermal relaxation and multiple cycles of photoswitching of piCRAC-1 (Figure S6) ....... S8

PiCRAC-1 enables light-inducible suppression of SOCE in living cells (Figure S7) S9

PiCRACs permits optical control over the nuclear translocation of NFAT1 downstream of the

CRAC channel (Figure S8). S11

Optical control of piCRAC-1 on thrombocyte progenitors in Tg (CD41:EGFP) zebrafish embryos (Figure S9)

Quantification of mRNA expression (Figure S10) S14

Synthesis and characterization of piCRACs S15

NMR, MS, HPLC Spectra of piCRACs. S34 

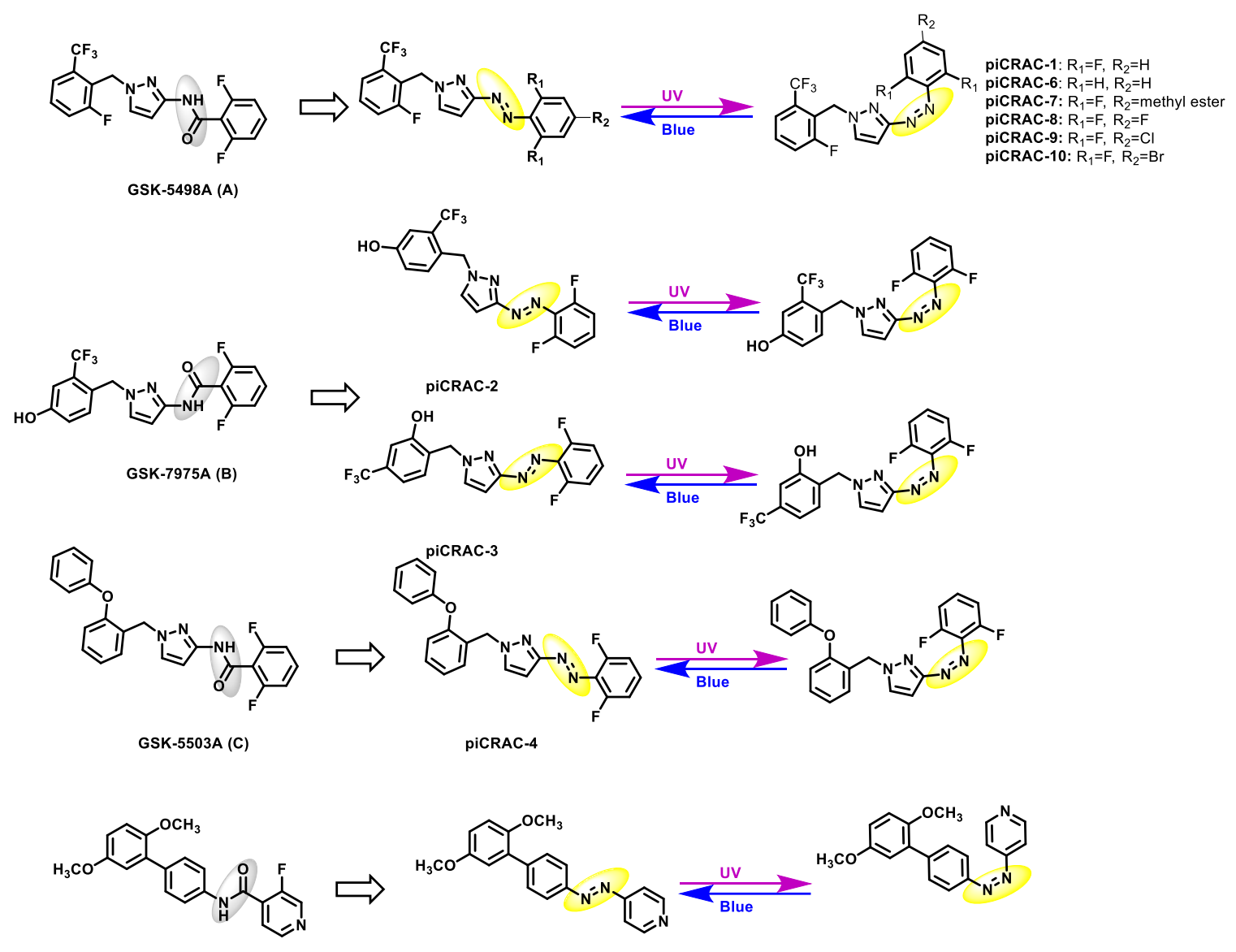

PICRAC-4

Synta 66 (D)

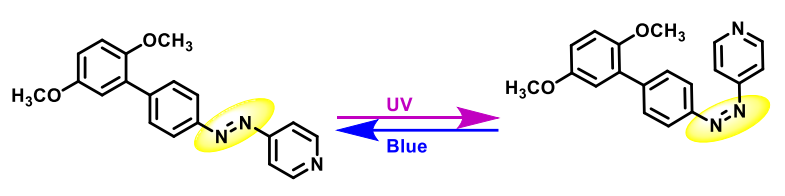

Figure S1. Design of photoswitchable CRAC channel inhibitors (piCRACs). The structures of the prototypic inhibitors, and the corresponding piCRACs with the incorporation of a photo-convertible azobenzene moiety were shown. 
a

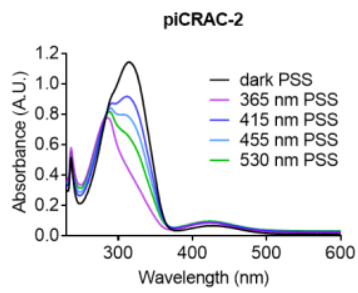

d

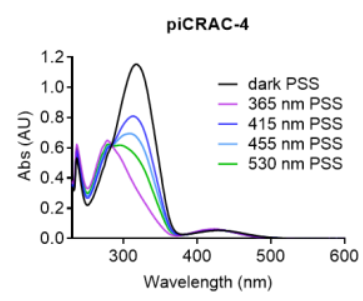

g

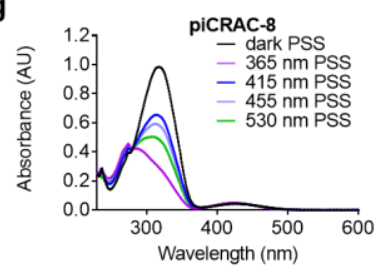

j

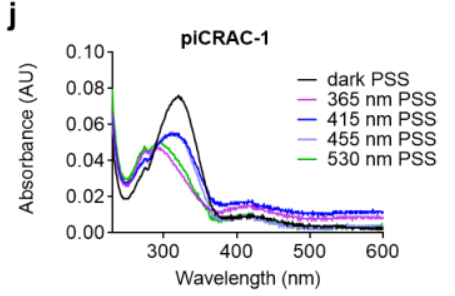

b

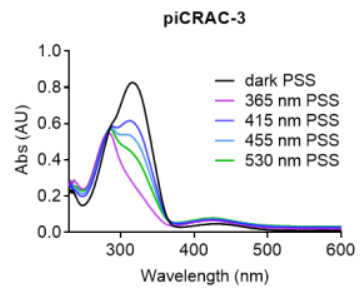

e

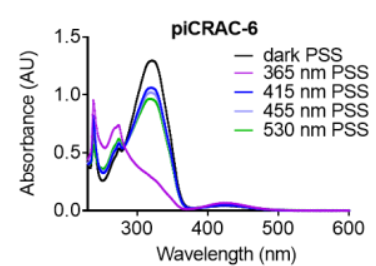

h

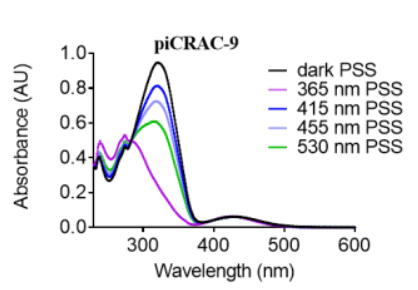

c

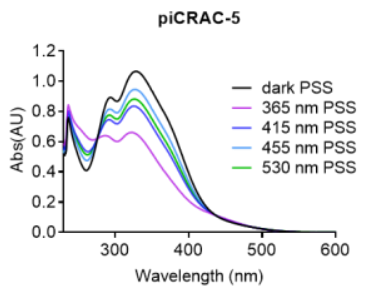

f

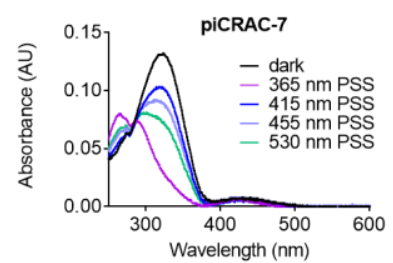

i

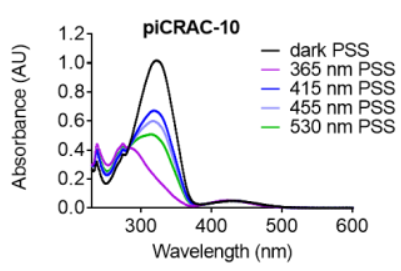

Figure S2. Photochemical characterization of piCRACs compounds. Shown were UV-Vis spectra of piCRAC-2 to piCRAC-10 (a-i) $(50 \mu \mathrm{M})$ prior to UV illumination (Dark), $365 \mathrm{~nm}$ PSS (9.8 mW), $415 \mathrm{~nm}$ PSS (14.4 mW), $455 \mathrm{~nm}$ PSS (9.5 mW) and $530 \mathrm{~nm}$ PSS (6.8 mW) in acetonitrile containing 0.5\% DMSO, respectively. j, UV-Vis spectra of piCRAC-1 $(10 \mu \mathrm{M})$ in Dark, or after illumination at $365 \mathrm{~nm}$ PSS (9.8 mW), $415 \mathrm{~nm}$ PSS (14.4 mW), $455 \mathrm{~nm}$ PSS (9.5 mW) and 530 nm PSS (6.8 mW) in E3 embryo medium containing 0.1\% DMSO. 

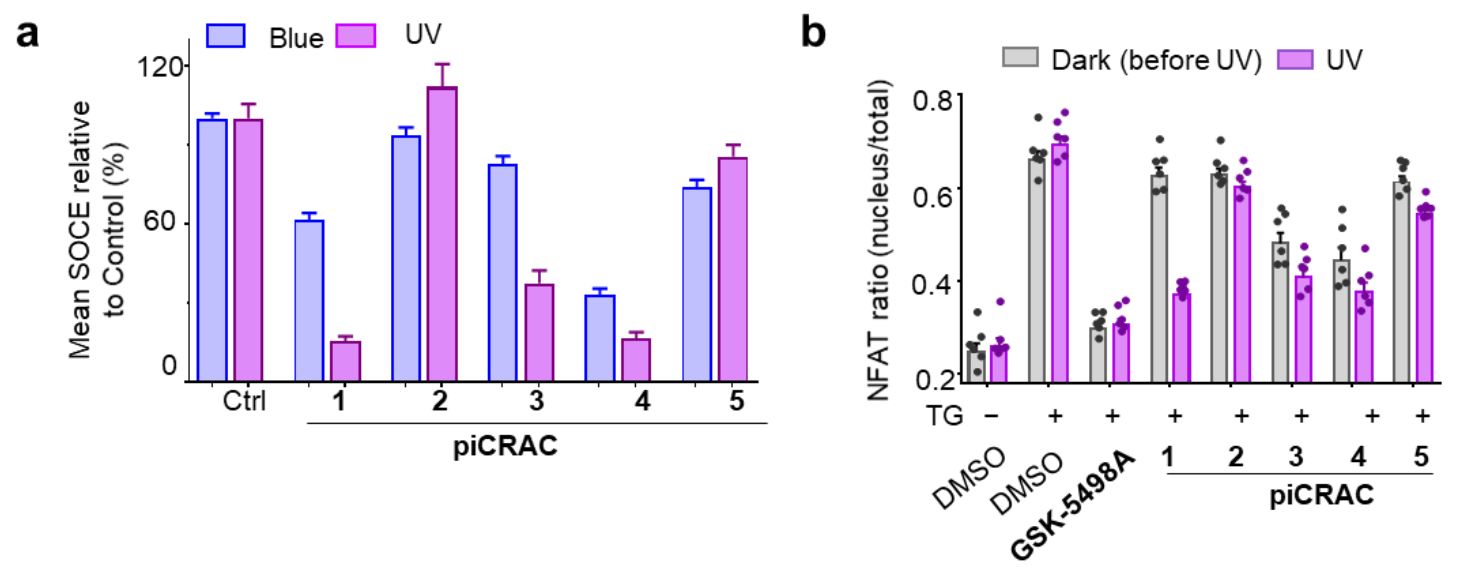

Figure S3. Light-inducible suppression on SOCE and NFAT1 nuclear translocation. a, Quantification of the effects of piCRAC-1 to $\mathbf{5}(10 \mu \mathrm{M})$ on SOCE in HEK 293 GEM-GECO cells under different light illumination conditions (UV, $365 \mathrm{~nm}$ or blue, $415 \mathrm{~nm}$ ). b, Quantification of the inhibitory activity of piCRAC compounds $(10 \mu \mathrm{M})$ against NFAT nuclear translocation in the dark or under $365 \mathrm{~nm}$ illumination. The inhibitory activity was indicated by the ratio of nucleus-overtotal NFAT1-GFP signals in each cell. $1 \mu \mathrm{M}$ TG was used to trigger $\mathrm{Ca}^{2+}$-influx and NFAT1 nuclear entry. Experiments were repeated three times with 200-300 cells analyzed. Error bars denote s.e.m.. 
a

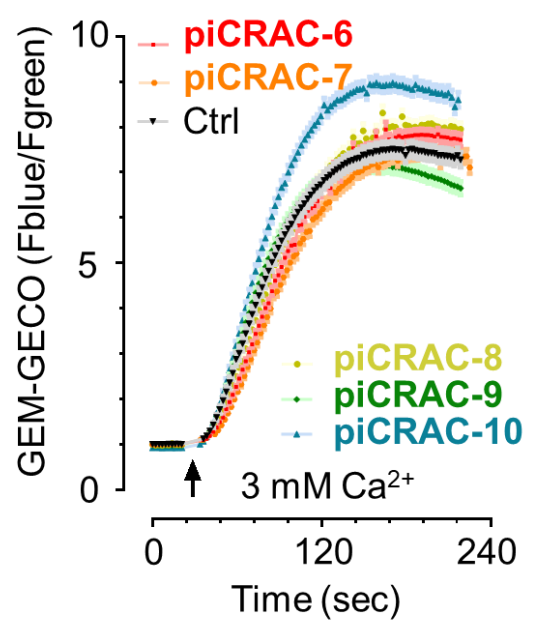

b

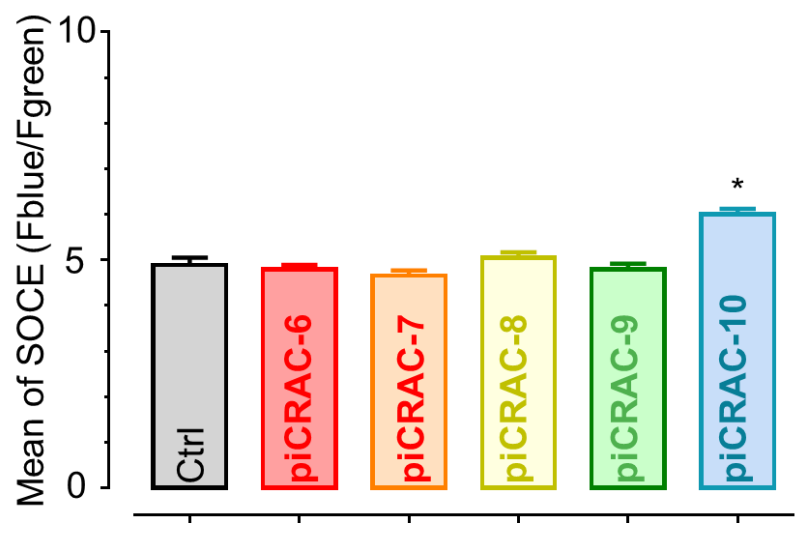

Figure S4. Effects of piCRAC6-10 on SOCE upon UV light illumination in HEK293 cells stably expressing GEM-GECO as a genetically encoded calcium indicator. a, The time courses of cytosolic $\mathrm{Ca}^{2+}$ signals reported by GEM-GECO for HEK293 cells treated with piCRACs 6-10. Before recordings, cells were bathed in $0 \mathrm{Ca}^{2+}$ imaging solution containing $1 \mu \mathrm{M}$ TG to deplete ER $\mathrm{Ca}^{2+}$ stores. Cells were bathed in a compound-containing solution for 2 min before recordings. 1 $\mu \mathrm{M}$ TG was present in the bath solution throughout recordings. b, Statistics of mean SOCE $(n=3$, Error bars denote s.e.m. $* P<0.05$, paired Student's t-test). 

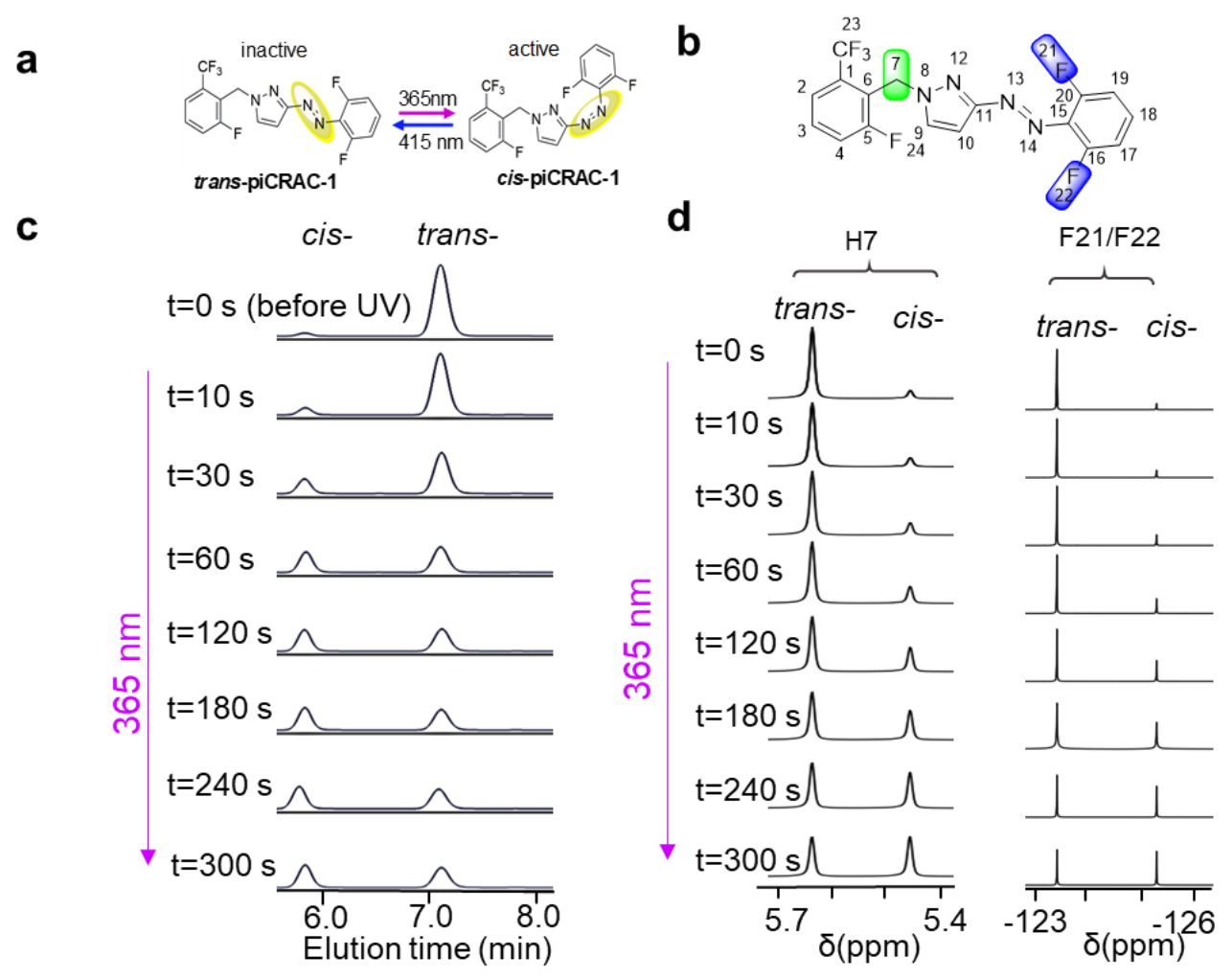

e
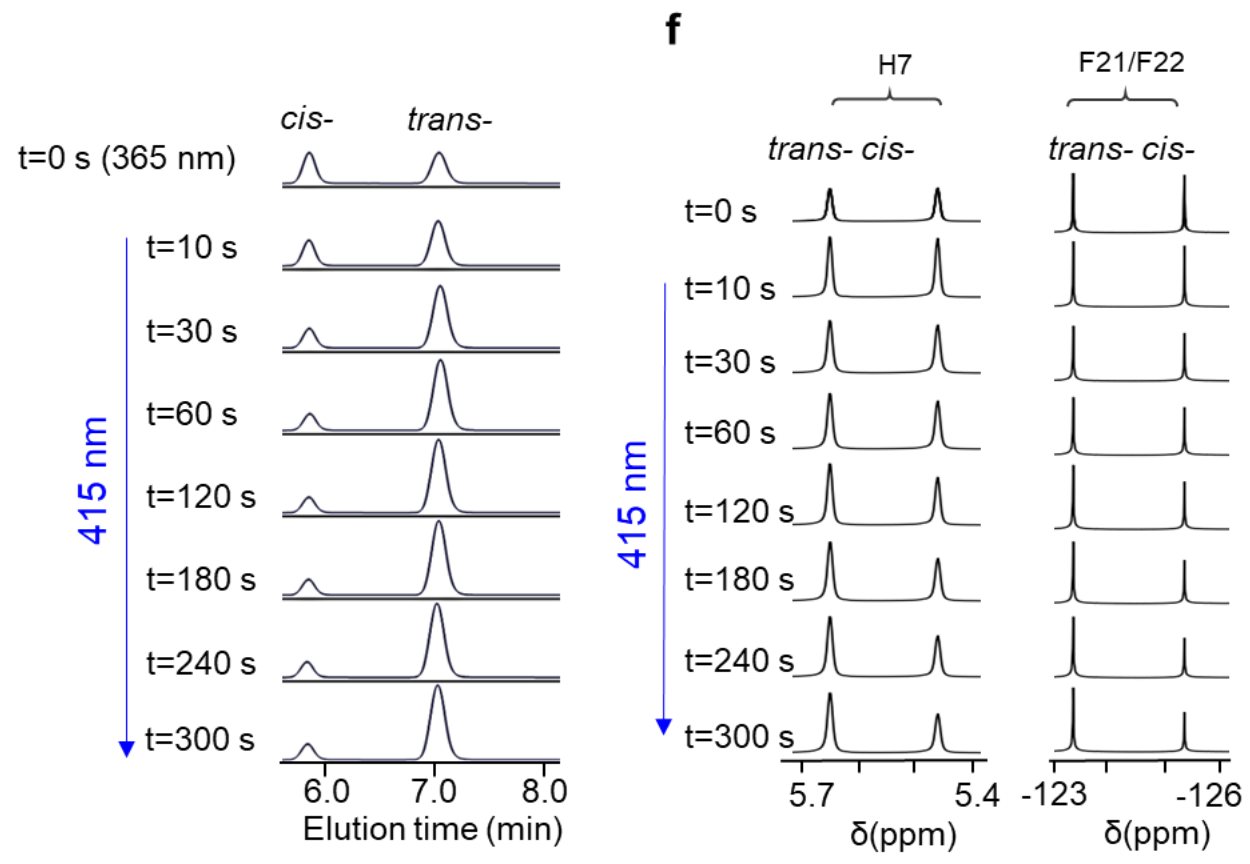

Figure S5. Photochemical characterization of piCRAC-1 by HPLC and NMR. a-b, PiCRAC1 photoisomerizes from its trans- to cis-isomer, and vice versa, in response to UV and blue light stimulation (a). To aid the assignment of NMR resonance peaks, the atom numbers of piCRAC-1 were labeled (b). c-d, HPLC elution profiles $(\mathbf{c}, 50 \mu \mathrm{M})$ and ${ }^{1} \mathrm{H} /{ }^{19} \mathrm{~F}-\mathrm{NMR}$ spectra $(\mathbf{d}, 35.39 \mathrm{mM})$ reporting the isomerization of trans-piCRAC-1 into cis-piCRAC-1 with illumination at $365 \mathrm{~nm}$ 
for $5 \mathrm{~min}$. Traces at time points 0, 30, 60, 120, and $300 \mathrm{~s}$ were also shown in Figure 2c-d. e-f, HPLC elution profiles in acetonitrile containing 0.5\% DMSO (e, $50 \mu \mathrm{M})$ and ${ }^{1} \mathrm{H} /{ }^{19} \mathrm{~F}-\mathrm{NMR}$ spectra in $\mathrm{MeOD}-d_{4}(\mathbf{f}, 35.39 \mathrm{mM})$ monitoring the isomerization of cis-piCRAC-1 to trans-piCRAC-1 with illumination at $415 \mathrm{~nm}$ for $5 \mathrm{~min}$. 
a
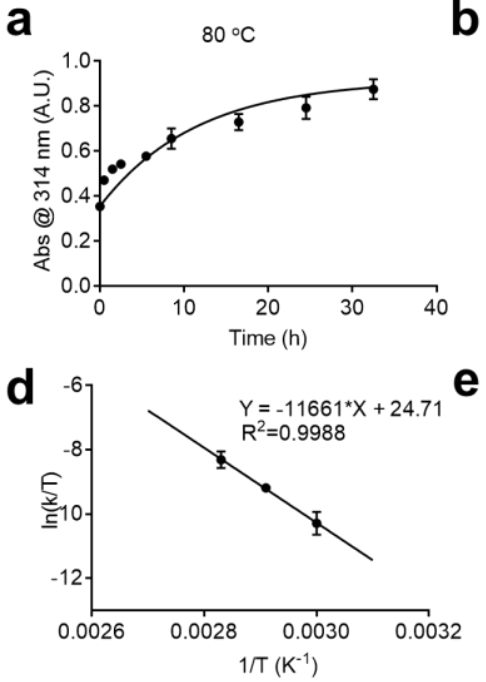

b
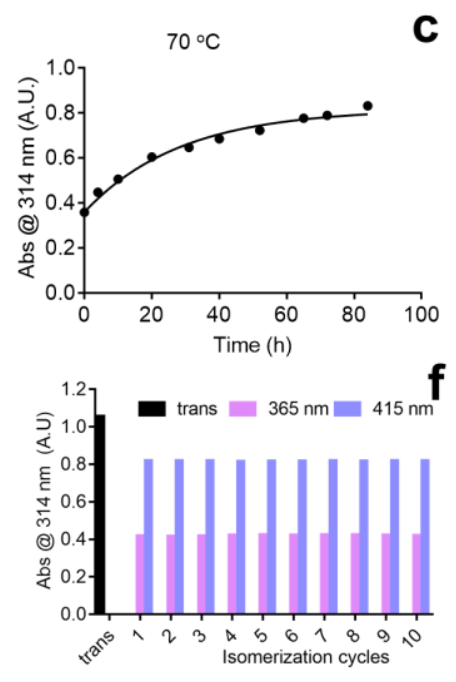

C
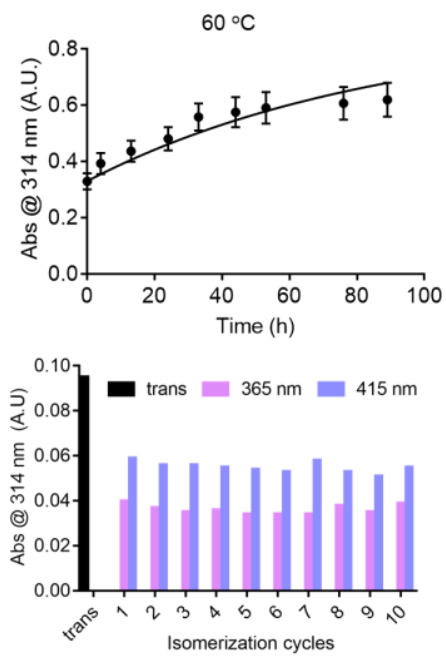

Figure S6. Thermal relaxation and multiple cycles of photoswitching of piCRAC-1. a-c, Thermal isomerization of piCRAC-1 at a range of temperatures in acetonitrile containing $0.5 \%$ DMSO. After converting trans-piCRAC-1 to cis-piCRAC-1 by illumination with $365 \mathrm{~nm}$ light for $5 \mathrm{~min}$. Absorbance at $314 \mathrm{~nm}$ was recorded to detect thermal stability. The solid line represents a fitted mono-exponential time course of data. d, Arrhenius plot of piCRAC-1 according to the thermal isomerization rate at $80,70,60^{\circ} \mathrm{C}$. e-f, Quantitative analysis of reversible photoisomerism of piCRAC-1 after repeatedly alternating the light stimulation between $365 \mathrm{~nm}$ and $415 \mathrm{~nm}$ in acetonitrile containing $0.5 \%$ DMSO (50 $\mu \mathrm{M}$; e) and in E3 culture solution containing $0.1 \%$ DMSO $(10 \mu \mathrm{M} ; \mathbf{f})$. The absorbance at $314 \mathrm{~nm}$ was used as readout. Three independent experiments were performed. $\mathbf{a}, \mathbf{b}$ and $\mathbf{c}$ error bars denote s.e.m.. 


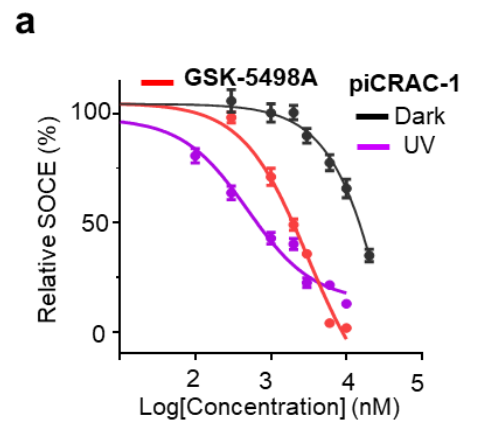

d

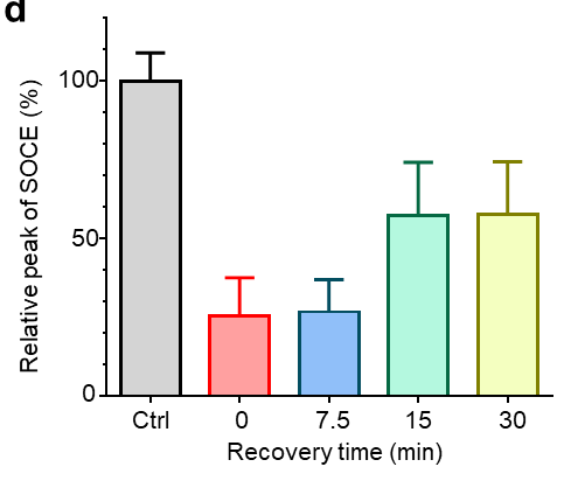

f

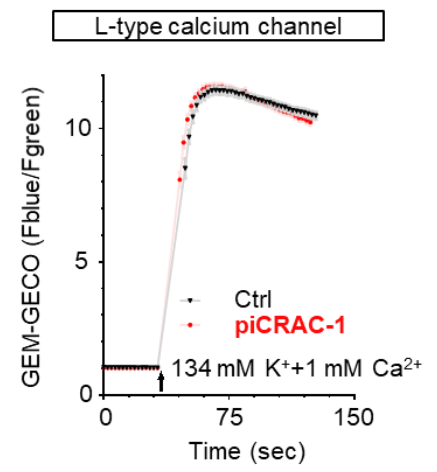

b

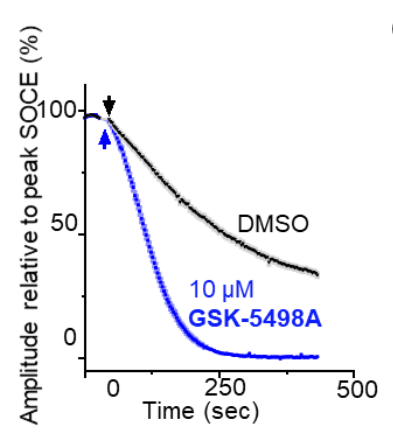

C

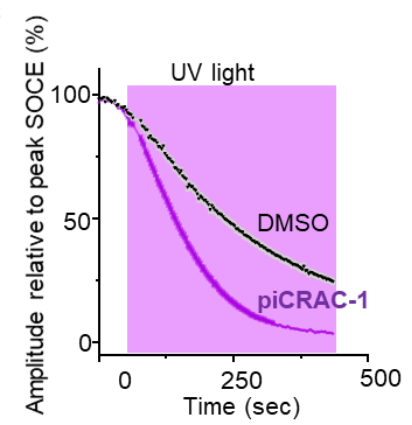

e

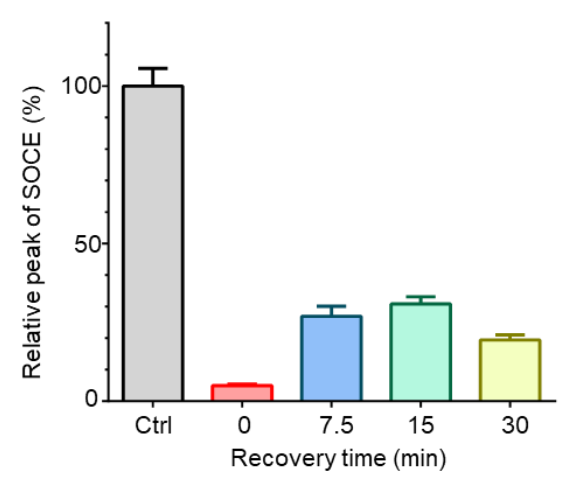

g

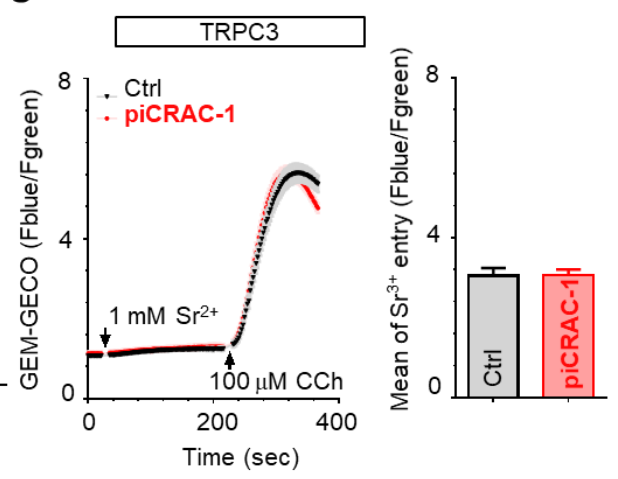

$\mathbf{h}$
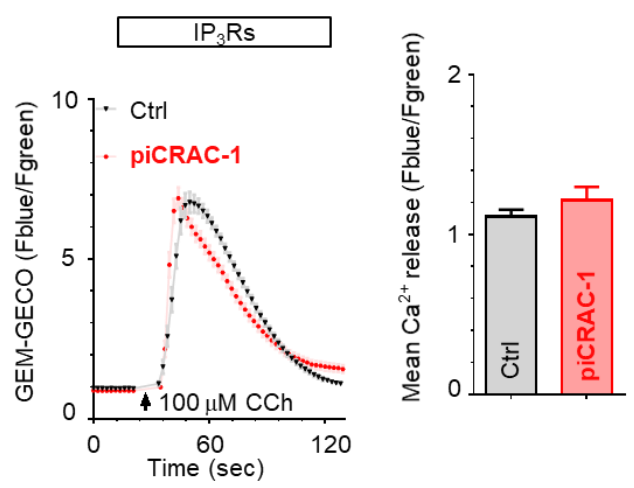

Figure S7. The effects of piCRAC-1 and its prototype, GSK-5498A, on SOCE and $\mathrm{Ca}^{2+}$ responses mediated by other $\mathrm{Ca}^{2+}$ channels. a, Dose-response curves of piCRAC-1 (trans- [dark] or cis-isomers [UV, violet]) and GSK-5498A (red). TG-triggered SOCE in HEK GEM-GECO cells 
was used as the readout. Cells were bathed in a compound-containing solution for 2 min before recordings. The curves of piCRAC-1 were also shown in Figure 2a. b-c, Comparison of the in-hibitory rate of piCRAC-1 and GSK-5498A on SOCE. $10 \mu \mathrm{M}$ GSK-5498A (b, blue curve) or piCRAC-1 (c, violet curve) were added into HEK cells. Upon addition of inhibitors or DMSO, the amplitudes of SOCE will decay as in the DMSO condition, the addition of CRAC channel inhibitors was anticipated to accelerate the decay as seen with GSK-5498A and photoactivated piCRAC-1. $365 \mathrm{~nm}$ illumination was applied in panel c. $\mathrm{n}=3$ independent experiments with at least 60 cells examined for each compound. d, Bar graph showing SOCE responses for cells incubated with cis-piCRAC-1 after blue light illumination at $415 \mathrm{~nm}$. Cells incubated with piCRAC-1 have first ex-posed to UV illumination $365 \mathrm{~nm}$ for $5 \mathrm{~min}$ and then subjected to 415 $\mathrm{nm}$ illumination at the indi-cated duration of time before SOCE responses were recorded. $\mathbf{e}$, Bar graph showing SOCE recovery for cells incubated with GSK-5498A after wash-out. f-h, The effects of UV-excited piCRAC-1 $\left(10 \mu \mathrm{M}, 5\right.$ min UV-illumination before recordings) on $\mathrm{Ca}^{2+}$ responses mediated by $\mathrm{Cav}_{1.2}$, TRPC3, and $\mathrm{IP}_{3} \mathrm{Rs}$ channels. Cells were transiently transfected with the calcium indicator GEM-GECO. Panels on the left, typical traces; panels on the right, statistics. f, HEK Cav stable cells. g, HEK-T365 cells stably expressing TRPC3. h, HEK wt cells with endogenous IP3Rs.Three independent repeats for each condition. Error bars denote s.e.m.. 

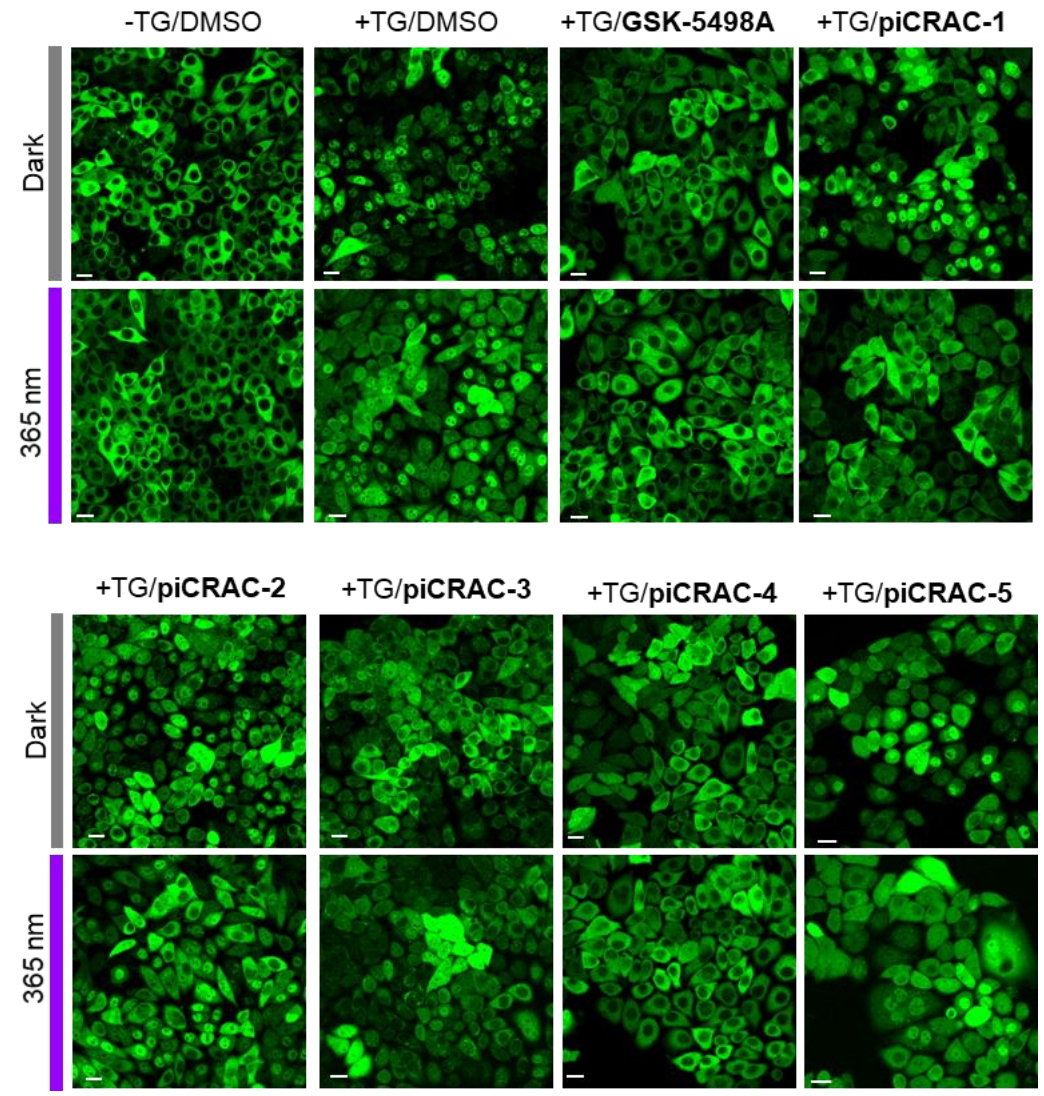

Figure S8. PiCRACs permits optical control over the nuclear translocation of NFAT1 downstream of the CRAC channel. Representative confocal images showing the effect of DMSO and piCRAC compounds 1-5 on NFAT1 nuclear translocation in the dark (prior to photoactivation) or under UV light illumination. $1 \mu \mathrm{M}$ TG was added to trigger $\mathrm{Ca}^{2+}$ store depletion and NFAT1 nuclear entry. Part of the data (DMSO and piCRAC-1 groups) were also used in Figure 2d-e to generate the enlarged images and the bar graph. Scale bar, $20 \mu \mathrm{m}$. Three times experiments repeated, 60-100 cells were measured in one image. 
a

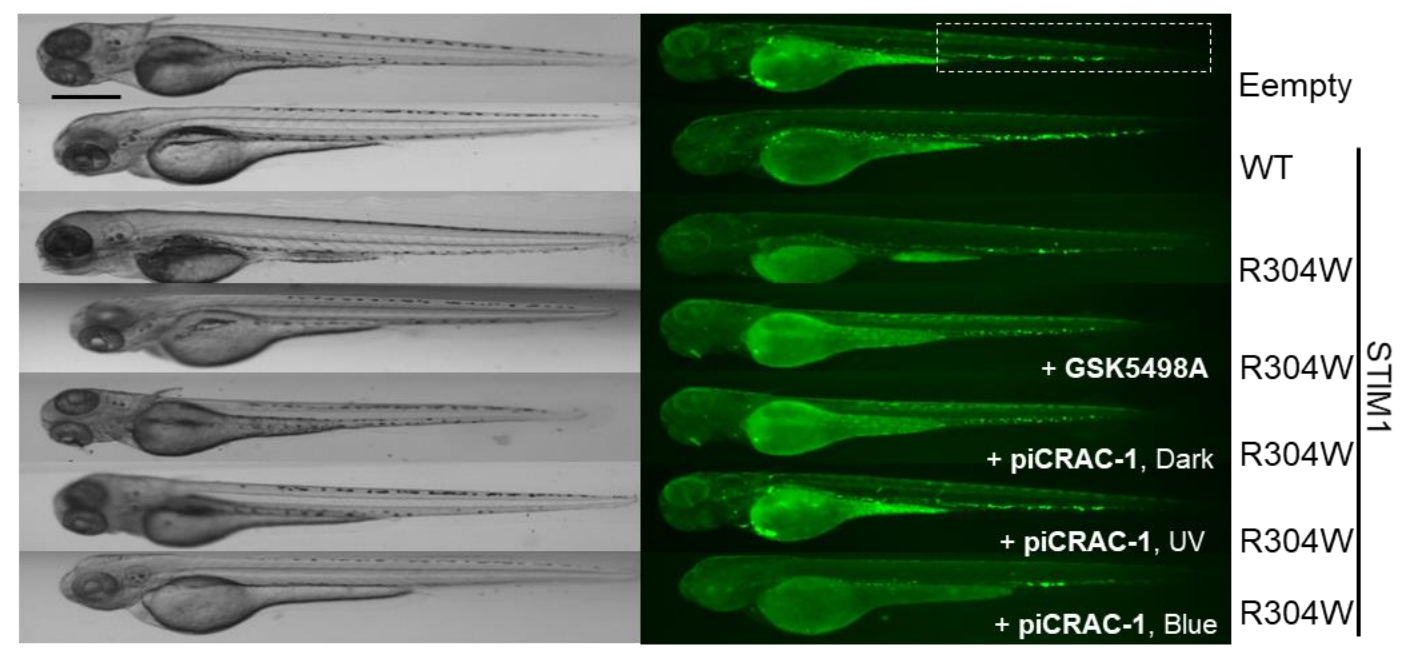

b

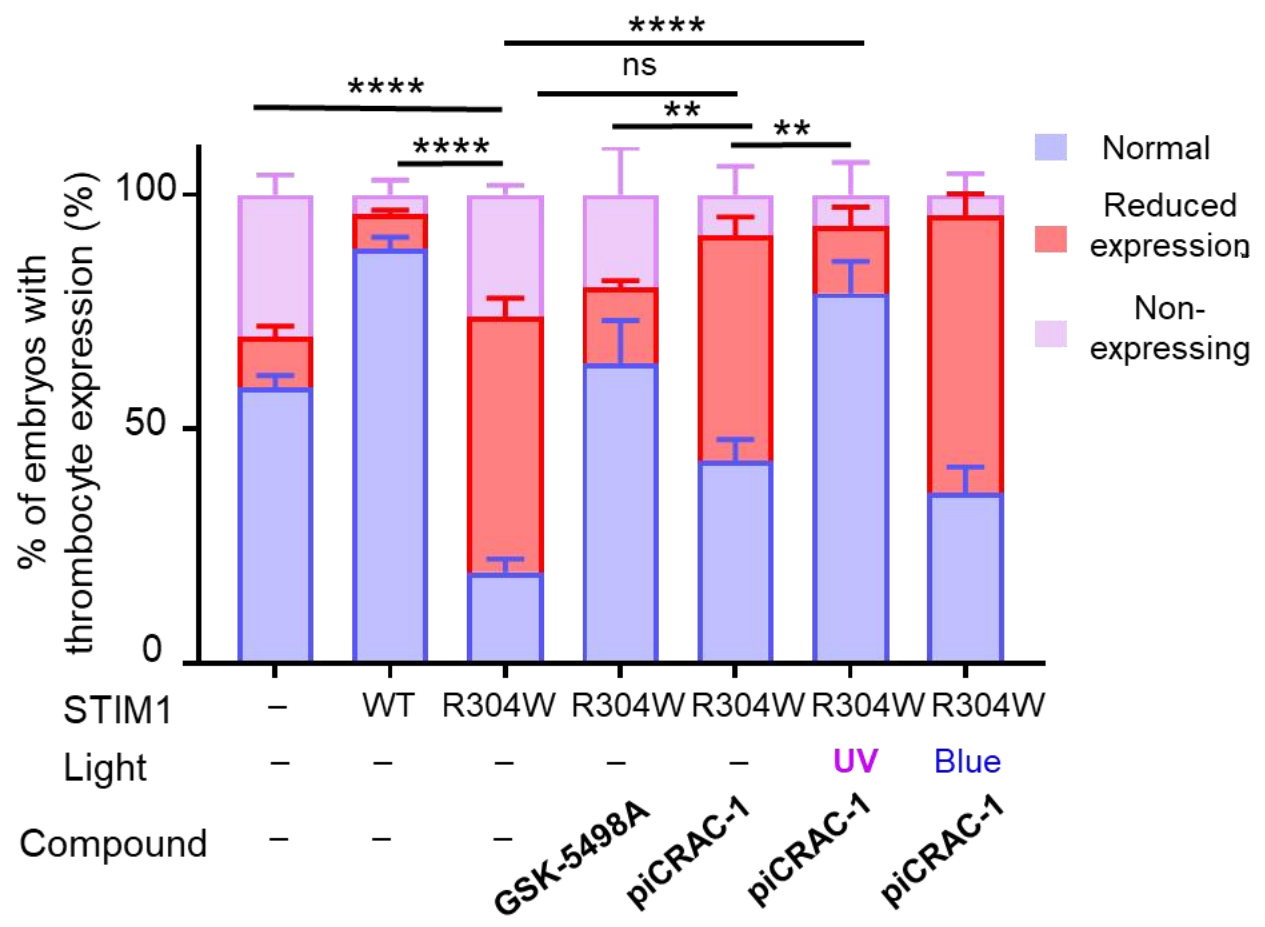

Figure S9 Optical control of piCRAC-1 on thrombocyte progenitors in Tg (CD41:EGFP) zebrafish embryos. a, Whole-body lateral views of Tg (CD41:EGFP) zebrafish embryos at the indicated conditions. Embryos were expressed differently capped mRNAs: empty vector as a control, STIM1(WT) and STIM1 (R304W), respectively. Embryos expressing the STIM1 mutant $\mathrm{R} 304 \mathrm{~W}$ that causes Storkormen syndrome in humans. The corresponding mRNAs were injected into the embryos. Developing embryos were either kept in the dark and under UV (365 nm) or blue light illumination (415 nm) for $5 \mathrm{~min}$ then raised for $2.5 \mathrm{~h} .5 \mu \mathrm{M}$ of piCRAC-1 or GSK-5498A was used. 25-78 embryos were counted per experiment with at least three independent replicates. Left, bright field; Right, green field with excitation at $488 \mathrm{~nm}$ to monitor GFP signals. Scale bar, 
$200 \mu \mathrm{m}$. b. The percentage of Tg (CD41:EGFP) zebrafish embryos with normal (blue), reduced (red) or no GFP expression (violet) in the thrombocyte progenitors (quantified from panel a). Three independent experiments were performed, with 25-78 embryos per experiment. The data were shown as mean \pm s.e.m. $(* * P<0.01$, $* * * * P<0.0001$, parametric, two-tailed, unpaired Student's $t$ test). 


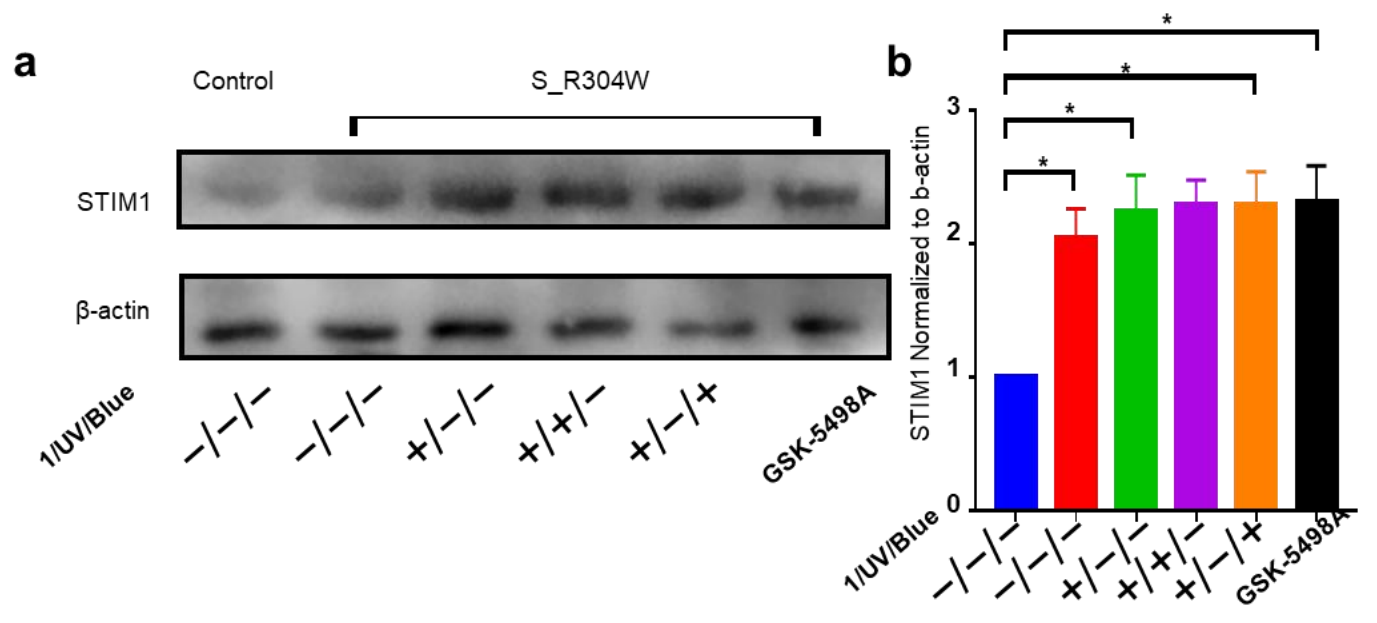

Figure S10 Western blotting analysis of STIM1 protein expression in zebrafish embryos. a, western blot of protein extracts from embryos injected with PBS and STIM1_R304W mRNA treated with piCRAC-1 $(10 \mu \mathrm{M})$ or GSK-5498A $(10 \mu \mathrm{M})$. b, Quantification of immunoblot bands corresponding to the indicated conditions. Protein expression values from a were normalized to the levels of $\beta$-actin. Data were shown as mean \pm s.e.m. ( $* P<0.05$ two-tailed unpaired Student's ttest, three-independent experiments). 1, piCRAC-1; UV: $365 \mathrm{~nm}$ light stimulation; Blue: $415 \mathrm{~nm}$ light illumination. 


\section{Synthesis and characterization of piCRACs.}

\section{1,3-difluoro-2-nitrosobenzene (1-2)}<smiles>O=[N+]([O-])c1c(F)cccc1F</smiles>

2,6-difluoroaniline (1-1, $1 \mathrm{~g}, 7.75 \mathrm{mmol}, 1.0$ equiv) and potassium peroxomonosulfate $(28.58 \mathrm{~g}$, $46.49 \mathrm{mmol}, 6.0$ equiv, $4.5 \%$ active oxygen) were stirred with a $\mathrm{CH}_{2} \mathrm{Cl}_{2} / \mathrm{H}_{2} \mathrm{O}(100 \mathrm{~mL}: 100 \mathrm{~mL}) \mathrm{mix}-$ ture solution at r.t. The reaction was stirred for $24 \mathrm{~h}$ and separated in an organic layer. The aqueous layer was extracted with dichloromethane $(10 \mathrm{~mL} * 2)$, and the combined organic layers were dried by $\mathrm{Na}_{2} \mathrm{SO}_{4}$. The solvent was evaporated in vacuo to yield green oil (1-2, $\left.0.96 \mathrm{~g}, 86.49 \%\right)$. The product was directly used in the next step without future purification.

TLC (PE): $\mathbf{R}_{\mathbf{f}}=0.9$

(E)-3-((2,6-difluorophenyl)diazenyl)-1H-pyrazole (1-3)<smiles>Fc1cccc(F)c1N=Nc1cc[nH]n1</smiles>

1,3-difluoro-2-nitrosobenzene (1-2, $0.96 \mathrm{~g}, 6.71 \mathrm{mmol}, 1.0$ equiv) and 1H-pyrazol-3-amine (6.71 mmol, 1.0 equiv) were dissolved in glacial acetic acid $(20 \mathrm{~mL})$ and stirred at r.t overnight. The yellow mixture became dark black. Glacial acetic acid was removed under reduced pressure, and the $\mathrm{pH}$ was adjusted to alkaline by $\mathrm{NaOH}(\mathrm{aq})$. The aqueous layer was extracted with $\mathrm{EA}(2 \mathrm{X} 20$ $\mathrm{mL})$. The combined organic layers were washed with brine $(5 \mathrm{~mL})$, separated and dried over sodium sulfate. The reaction was condensed and purified via column chromatography (PE:EA $=2: 1$ ) to yield yellow solid (1-4, 0.35g, 25.17\%).

m.p: $164.3-167.7^{\circ} \mathrm{C}$.

TLC (PE:EA 3:1): $\mathbf{R}_{\mathbf{f}}=0.3$

${ }^{1}$ H NMR (400 MHz, MeOD-d $\left.\boldsymbol{d}_{4}\right): \delta 7.75(\mathrm{~d}, J=2.3 \mathrm{~Hz}, 1 \mathrm{H}), 7.46(\mathrm{dt}, J=20.3,10.2 \mathrm{~Hz}, 1 \mathrm{H}), 7.15$ (t, $J=8.9 \mathrm{~Hz}, 2 \mathrm{H}), 6.65(\mathrm{~s}, 1 \mathrm{H})$.

${ }^{13}$ C NMR (101 MHz, MeOD-d $\left.\boldsymbol{d}_{4}\right): \delta 156.88$ (d, $\left.J=4.3 \mathrm{~Hz}, 1 \mathrm{C}\right), 154.31(\mathrm{~d}, J=4.2 \mathrm{~Hz}, 1 \mathrm{C}), 130.87$ (t, $J=10.9 \mathrm{~Hz}, 1 \mathrm{C}), 130.43$ (s, 1C),112.45 - 112.31 (m, 1C), $112.23-112.12$ (m, 1C), 93.23 (s, 1C).

ESI-HRMS: $m / z$ calcd. for $\left[\mathrm{C}_{9} \mathrm{H}_{5} \mathrm{~F}_{2} \mathrm{~N}_{4}\right]^{-}: 207.0488$, found $207.0649\left([\mathrm{M}-\mathrm{H}]^{-}\right)$.

\section{(E)-3-((2,6-difluorophenyl)diazenyl)-1-(2-fluoro-6-(trifluoromethyl)benzyl)-1H-pyrazole (piCRAC-1)}




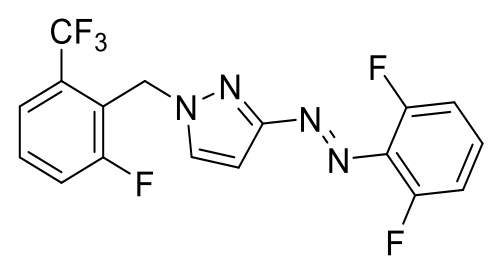

PICRAC-1

(E)-3-((2,6-difluorophenyl)diazenyl)-1H-pyrazole (1-3, $0.10 \mathrm{~g}, 0.48 \mathrm{mmol}, 1.0$ equiv) was dissolved in dry THF $(20 \mathrm{~mL})$, purged with $\mathrm{N}_{2}$ (twice) prior to use and carried out under an atmosphere of $\mathrm{N}_{2}$. Lithium bis(trimethylsiy)amide ( $0.96 \mathrm{~mL}$ ( $1 \mathrm{M} / \mathrm{L}$ in THF), $0.96 \mathrm{mmol}, 2.0$ equiv) was added dropwise under the presence of $\mathrm{N}_{2}$. After $0.5 \mathrm{~h}$, 2-(bromomethyl)-1-fluoro-3-(trifluoromethyl)benzene( $0.18 \mathrm{~g}, 0.72 \mathrm{mmol}, 1.5 \mathrm{equiv})$ was added under nitrogen protection. The mixture was stirred overnight, and quenched with sat. $\mathrm{NH}_{4} \mathrm{Cl}(\mathrm{aq}, 20 \mathrm{~mL}$ ), The aqueous layer was extracted with EA (20 mLX1, $5 \mathrm{mLX} 2)$. The combined organic phase was separated, condensed and dried with $\mathrm{Na}_{2} \mathrm{SO}_{4}$, yielding (E)-3-((2,6-difluorophenyl)diazenyl)-1-(2-fluoro-6-(trifluoromethyl)benzyl)1H-pyrazole (piCRAC-1, $136 \mathrm{mg}, 73.67 \%$, Purity of trans $94.97 \%$, Sig = $290 \mathrm{~nm}, 4 \mathrm{~nm}$ ). m.p: $72.3-78.9^{\circ} \mathrm{C}$.

TLC (PE:EA 10:1): $\mathbf{R}_{\mathbf{f}}=0.4$.

${ }^{1}$ H NMR (400 MHz, MeOD-d d $^{\delta} 7.69-7.63(\mathrm{~m}, 3 \mathrm{H}), 7.60-7.49(\mathrm{~m}, 1 \mathrm{H}), 7.48-7.37(\mathrm{~m}, 1 \mathrm{H})$, $7.13(\mathrm{t}, J=8.9 \mathrm{~Hz}, 2 \mathrm{H}), 6.60(\mathrm{~d}, J=2.3 \mathrm{~Hz}, 1 \mathrm{H}), 5.67(\mathrm{~s}, 2 \mathrm{H})$.

${ }^{13}$ C NMR (101 MHz, MeOD-d $\left.d_{4}\right) \delta 164.31(\mathrm{~s}), 162.54(\mathrm{~d}, \mathrm{~J}=250.1 \mathrm{~Hz}), 156.85(\mathrm{~d}, \mathrm{~J}=4.2 \mathrm{~Hz})$, 154.28 (d, J = 4.2 Hz), 132.03 (s), 131.39 (d, J = 9.5 Hz), 130.91 (t, J = 10.4 Hz), 127.86 (s), 124.95 (d, J = 4.0 Hz), 122.46 - $121.66(\mathrm{~m}), 120.26$ (s), 119.90 (d, J = 22.8 Hz), 112.44 - 112.30 (m), $112.22-112.10(\mathrm{~m}), 94.27(\mathrm{~s}), 46.13(\mathrm{~s})$.

${ }^{19}$ F NMR (376 MHz, MeOD-d 4 ) $\delta-59.90$ (s, 3F), -114.10 (s, 1F), -123.29 (s, 2F).

ESI-HRMS: $m / z$ calcd. for $\left[\mathrm{C}_{17} \mathrm{H}_{11} \mathrm{~F}_{6} \mathrm{~N}_{4}\right]^{+}: 385.0882$, found $385.0885\left([\mathrm{M}+\mathrm{H}]^{+}\right), \mathrm{m} / z$ calcd. for $\left[\mathrm{C}_{17} \mathrm{H}_{10} \mathrm{~F}_{6} \mathrm{~N}_{4} \mathrm{Na}\right]^{+}:$407.0702, found $405.0706\left([\mathrm{M}+\mathrm{Na}]^{+}\right)$.

HPLC: mobile phase acetonitrile: $\mathrm{H}_{2} \mathrm{O}$ (75:25), flow velocity $(1.0 \mathrm{~mL} / \mathrm{min}) . \mathrm{Sig}=290 \mathrm{~nm}, 4 \mathrm{~nm}$, purity of trans $94.97 \%$.

\section{4-(hydroxymethyl)-3-(trifluoromethyl)phenol (2-2)}<smiles>OCc1ccc(O)cc1C(F)(F)F</smiles>

4-hydroxy-2-(trifluoromethyl)benzoic acid (2-1, $2 \mathrm{~g}, 9.71 \mathrm{mmol}, 1.0$ equiv) was dissolved in dry THF (20mL), and $\mathrm{LiAlH}_{4}(0.74 \mathrm{~g}, 1.94 \mathrm{mmol}, 2$ equiv) was added in portion under ice bath for 30 min. The mixture generated a large number of bubbles and became gray-black. The reaction was heated at $66^{\circ} \mathrm{C}$ for $1.5 \mathrm{~h}$, the suspension was cooled to $0{ }^{\circ} \mathrm{C}$, and quenched with saturated citric acid solution to adjust the $\mathrm{pH}$ to 4 . After $5 \mathrm{~min}$, the suspension was filtered through celite, the 
filtrate was extracted with EA (50 mLX1, $10 \mathrm{~mL} \mathrm{X2)}$. and the organic phase was combined and dried by $\mathrm{Na}_{2} \mathrm{SO}_{4}$. The solvent was evaporated in vacuo and the product purified via column chromatography (80 $\left.\mathrm{g} \mathrm{SiO}_{2}, \mathrm{PE}: \mathrm{EA}=2: 1\right)$ to yield white solid (2-2, $\left.0.83 \mathrm{~g}, 41.03 \%\right)$.

m.p: $91.2-93.7^{\circ} \mathrm{C}$.

TLC (PE:EA 3:1): $\mathbf{R}_{\mathbf{f}}=0.6$

${ }^{1}$ H NMR (400 MHz, CD ${ }_{3}$ OD- $\left.d_{4}\right): \delta 7.51(\mathrm{~d}, J=8.4 \mathrm{~Hz}, 1 \mathrm{H}$, ), $7.04(\mathrm{~d}, J=2.4 \mathrm{~Hz}, 1 \mathrm{H}), 7.00$ (dd, $J=8.4,2.1 \mathrm{~Hz}, 1 \mathrm{H}), 4.67$ (s, 2H).

${ }^{13}$ C NMR (101 MHz, CD ${ }_{3}$ OD-d $\left.d_{4}\right): \delta 156.62$ (s, 1C), 130.58 (s, 1C), 129.91 (s, 1C), 127.97 (dd, $J$ $=61.7,31.1 \mathrm{~Hz}, 1 \mathrm{C}), 124.36(\mathrm{dd}, J=273.1 \mathrm{~Hz}, 1 \mathrm{C}), 118.29$ (s, 1C), 112.11 (q, $J=5.8 \mathrm{~Hz}, 1 \mathrm{C})$, $59.60(\mathrm{dd}, J=5.5,2.7 \mathrm{~Hz}, 1 \mathrm{C})$.

ESI-HRMS: $m / z$ calcd. for $\left[\mathrm{C}_{8} \mathrm{H}_{6} \mathrm{~F}_{3} \mathrm{O}_{2}\right]^{-:}: 191.0325$, found $191.0483\left([\mathrm{M}-\mathrm{H}]^{-}\right)$.

\section{(4-(benzyloxy)-2-(trifluoromethyl)phenyl)methanol (2-3)}<smiles>OCc1ccc(OCc2ccccc2)cc1C(F)(F)F</smiles>

2-3

4-(hydroxymethyl)-3-(trifluoromethyl)phenol (2-2, $0.75 \mathrm{~g}, 3.91 \mathrm{mmol}, 1.0$ equeiv), (bromomethyl)benzene ( $1.33 \mathrm{~g}, 7.81 \mathrm{mmol}, 2.0$ equiv) and sodium hydroxide $(0.47 \mathrm{~g}, 11.72 \mathrm{mmol}, 3.0$ equiv) was mixed in acetonitrile $(20 \mathrm{~mL})$. The reaction was stirred at ambient temperature for $6 \mathrm{~h}$. The solvent was evaporated in vacuo. The residue was extracted with 3:1 EA: $\mathrm{H}_{2} \mathrm{O}(20 \mathrm{~mL}: 20 \mathrm{~mL})$. The aqueous phase was extracted with $\mathrm{EA}(10 \mathrm{mLx} 2)$, the combined organic phase was dried by $\mathrm{Na}_{2} \mathrm{SO}_{4}$. After $2 \mathrm{~h}, \mathrm{Na}_{2} \mathrm{SO}_{4}$ was filtered, the filtrate was condensed and further purified via column chromatography $\left(40 \mathrm{~g}, \mathrm{SiO}_{2}, \mathrm{PE}: \mathrm{EA}=3: 1\right)$ to yield white solid $(\mathbf{2 - 3}, 0.75 \mathrm{~g}, 68.56 \%)$.

m.p: $60.7-61.1^{\circ} \mathrm{C}$.

TLC (PE:EA 3:1): $\mathbf{R}_{\mathbf{f}}=0.8$

${ }^{1}$ H NMR (400 MHz, MeOD-d $\left.)_{4}\right): \delta .63(\mathrm{~d}, J=9.1 \mathrm{~Hz}, 1 \mathrm{H}), 7.44(\mathrm{~m}, J=7.3 \mathrm{~Hz}, 2 \mathrm{H}), 7.37$ (t, $J=$ $7.4 \mathrm{~Hz}, 2 \mathrm{H}), 7.34-7.28(\mathrm{~m}, 1 \mathrm{H}), 7.23(\mathrm{~m}, J=6.9 \mathrm{~Hz}, 2 \mathrm{H}), 5.13(\mathrm{~s}, 2 \mathrm{H}), 4.70(\mathrm{~s}, 2 \mathrm{H})$.

${ }^{13}$ C NMR (101 MHz, MeOD-d 4 ): $\delta 157.80$ (s, 1C), 136.73 (s, 1C), 131.73 (s, 1C), 130.41 (s, 1C), 128.18 (s, 2C), 127.90 (dd, $J=61.0,30.1 \mathrm{~Hz}, 1 \mathrm{C}), 127.67$ (s, 1C), 127.24 (s, 2C), 124.70 (dd, $J=$ $554.6,281.3 \mathrm{~Hz}, 1 \mathrm{C}), 117.68$ (s, 1C), 112.33 (q, $J=5.9 \mathrm{~Hz}, 1 \mathrm{C}), 69.92$ (s, 1C), 59.47 (dd, $J=5.8$, $2.9 \mathrm{~Hz}, 1 \mathrm{C})$.

4-(benzyloxy)-1-(bromomethyl)-2-(trifluoromethyl)benzene (2-4) 
<smiles>FC(F)(F)c1cc(OCc2ccccc2)ccc1CBr</smiles>

$2-4$

(4-(benzyloxy)-2-(trifluoromethyl)phenyl)methanol (2-3, $0.3 \mathrm{~g}, 1.06 \mathrm{mmol}, 1.0$ equiv) was dissolved in DCM (20 mL) at $0{ }^{\circ} \mathrm{C}$ obtained solution A. Tribromophosphane $(0.39 \mathrm{~g}, 1.28 \mathrm{mmol}, 1.2$ equiv) was dissolved in DCM $(10 \mathrm{~mL})$ obtained solution B. B was added dropwise into A at $0{ }^{\circ} \mathrm{C}$ for $30 \mathrm{~min}$. Then, the cooling bath was removed and stirred for $1.5 \mathrm{~h}$. The reaction was monitored by TLC till 2-3 disappeared. Ice water $(20 \mathrm{~mL})$ and saturated sodium bicarbonate $(10 \mathrm{~mL})$ were subsequently added. The aqueous phase was extracted with DCM (10 mLx2). The combined organic layers were washed with brine $\left(10 \mathrm{~mL}^{*} 1\right)$, separated and dried over $\mathrm{Na}_{2} \mathrm{SO}_{4}$. Solvent was removed in vacuo to yield white oil (2-4, $0.18 \mathrm{~g}, 48.22 \%)$

TLC (PE:EA 10:1): $\mathbf{R}_{\mathbf{f}}=0.6$

ESI-MS: $m / z$ calcd. for $\left[\mathrm{C}_{15} \mathrm{H}_{11} \mathrm{BrF}_{3} \mathrm{O}\right]^{-:}: 344.99$, found $345.12\left([\mathrm{M}-\mathrm{H}]^{-}\right)$.

(E)-1-(4-(benzyloxy)-2-(trifluoromethyl)benzyl)-3-((2,6-difluorophenyl)diazenyl)-1H-pyrazole (2-5)<smiles>Fc1cccc(F)c1N=Nc1ccn(Cc2ccc(OCc3ccccc3)cc2C(F)(F)F)n1</smiles>

2-5

The preparation method of 2-5 was similar to piCRAC-1.

Then yellow oil product was isolated via column chromatography $(\mathrm{PE}: \mathrm{EA}=10: 1)(\mathbf{2 - 6}, 0.18$, $83.43 \%)$.

TLC (PE:EA 10:1): $\mathbf{R}_{\mathbf{f}}=0.4$.

ESI-HRMS: $m / z$ calcd. for $\left[\mathrm{C}_{24} \mathrm{H}_{18} \mathrm{~F}_{5} \mathrm{~N}_{4} \mathrm{O}\right]^{+}: 473.1395$, found $473.1374\left([\mathrm{M}+\mathrm{H}]^{+}\right) . \mathrm{m} / z$ calcd. for $\left[\mathrm{C}_{24} \mathrm{H}_{17} \mathrm{~F}_{5} \mathrm{~N}_{4} \mathrm{ONa}\right]^{+}$: 495.1215, found $495.1181\left([\mathrm{M}+\mathrm{Na}]^{+}\right)$. $\mathrm{m} / \mathrm{z}$ calcd. for $\left[\mathrm{C}_{24} \mathrm{H}_{17} \mathrm{~F}_{5} \mathrm{~N}_{4} \mathrm{OK}\right]^{+}$: 511.0954, found $511.0926\left([\mathrm{M}+\mathrm{K}]^{+}\right)$.

(E)-4-((3-((2,6-difluorophenyl)diazenyl)-1H-pyrazol-1-yl)methyl)-3-(trifluoromethyl)phenol (piCRAC-2) 
<smiles>Oc1ccc(Cn2ccc(/N=N/c3c(F)cccc3F)n2)c(C(F)(F)F)c1</smiles>

PICRAC-2

(E)-1-(4-(benzyloxy)-2-(trifluoromethyl)benzyl)-3-((2,6-difluorophenyl)diazenyl)-1H-pyrazole (2-5, $0.18 \mathrm{~g}, 0.38 \mathrm{mmol}, 1.0$ equiv) was dissolved with DCM $(20 \mathrm{~mL})$. Next, titanium tetrachloride ( $0.08 \mathrm{~g}, 0.42 \mathrm{mmol}, 1.1$ equiv) was drop wised the solution for $20 \mathrm{~min}$. After 30 minutes, the reaction was quenched with methanol $(10 \mathrm{~mL})$. The residue was extracted with ethyl acetate (30 mLx1, $10 \mathrm{mLx}$ ). PiCRAC-2 was purified via column chromatography (23 mg, 15.79\%, Purity of trans $96.79 \%$, Sig= $290 \mathrm{~nm}, 4 \mathrm{~nm}$ ).

m.p: $218.6-220.4^{\circ} \mathrm{C}$.

TLC (PE:EA 3:1): $\mathbf{R}_{\mathbf{f}}=0.5$

${ }^{1}$ H NMR (400 MHz, MeOD-d $\left.d_{4}\right): \delta 7.65(\mathrm{~d}, J=2.5 \mathrm{~Hz}, 1 \mathrm{H}), 7.45(\mathrm{ddd}, J=12.0,7.2,4.3 \mathrm{~Hz}, 1 \mathrm{H})$, $7.21-7.09(\mathrm{~m}, 4 \mathrm{H}), 7.01(\mathrm{dd}, J=8.4,2.4 \mathrm{~Hz}, 1 \mathrm{H}), 6.64(\mathrm{~d}, J=2.5 \mathrm{~Hz}, 1 \mathrm{H}), 5.55(\mathrm{~s}, 2 \mathrm{H})$.

${ }^{13}$ C NMR (101 MHz, MeOD-d d $_{\text {): } \delta} 164.35$ (s, 1C), 157.80 (s, 1C), 156.90 (d, $\left.J=4.3 \mathrm{~Hz}, 1 \mathrm{C}\right)$, 154.33 (d, $J=4.0 \mathrm{~Hz}, 1 \mathrm{C}), 132.44$ (s, 1C), 132.14 (2C), 130.96 (dd, $J=13.7,7.1 \mathrm{~Hz}, 1 \mathrm{C}), 130.77$ (s, 1C), 128.48 (dd, $J=272.3,241.6 \mathrm{~Hz}, 1 \mathrm{C}), 123.73$ (s, 1C), 118.81 (s, 1C), 112.81 (dd, $J=11.7$, $5.8 \mathrm{~Hz}, 1 \mathrm{C}), 112.47-112.32$ (m, 1C), $112.25-112.06$ (m, 1C), 94.61 (s, 1C), 52.24 (s, 1C).

ESI-HRMS: $m / z$ calcd. for $\left[\mathrm{C}_{17} \mathrm{H}_{12} \mathrm{~F}_{5} \mathrm{~N}_{4} \mathrm{O}\right]^{+}: 383.0926$, found $383.0958\left([\mathrm{M}+\mathrm{H}]^{+}\right), \mathrm{m} / z$ calcd. for $\left[\mathrm{C}_{17} \mathrm{H}_{11} \mathrm{~F}_{5} \mathrm{~N}_{4} \mathrm{ONa}\right]^{+}$: 405.0745, found $405.0777\left([\mathrm{M}+\mathrm{Na}]^{+}\right)$

HPLC: mobile phase acetonitrile: $\mathrm{H}_{2} \mathrm{O}(70: 30)$, flow velocity $(1.0 \mathrm{~mL} / \mathrm{min})$. $\mathrm{Sig}=290 \mathrm{~nm}, 4 \mathrm{~nm}$, purity of trans $96.79 \%$

2-(hydroxymethyl)-5-(trifluoromethyl)phenol (3-2)<smiles>OCc1ccc(C(F)(F)F)cc1O</smiles>

Following the general protocol of $\mathbf{2 - 2}$, It was to yield 2-(hydroxymethyl)-5-(trifluoromethyl)phenol(3-2, 0.45g, $48.39 \%$,

m.p: $64.3-67.3{ }^{\circ} \mathrm{C}$.

TLC (PE:EA 3:1): $\mathbf{R}_{\mathbf{f}}=0.65$

${ }^{1}$ H NMR (400 MHz, Methanol-d 4 ): $\delta 7.46(\mathrm{~d}, J=7.9 \mathrm{~Hz}, 1 \mathrm{H}), 7.10(\mathrm{~d}, J=7.9 \mathrm{~Hz}, 1 \mathrm{H}), 7.00$ (s, 1H), 4.68 (s, 2H).

${ }^{13}$ C NMR (101 MHz, MeOD-d $): \delta 154.91$ (s, 1C), 132.01 (s, 1C), 129.79 (q, $J=32.0 \mathrm{~Hz}, 1 \mathrm{C}$ ), 127.74 (s, 1C), 124.28 (dd, $J=542.2,271.2 \mathrm{~Hz}, 1 \mathrm{C}), 115.38$ (q, $J=4.0 \mathrm{~Hz}, 1 \mathrm{C}), 110.66$ (q, $J=3.7$ $\mathrm{Hz}, 1 \mathrm{C}), 58.87$ (s, 1C).

ESI-HRMS: $m / z$ calcd. for $\left[\mathrm{C}_{8} \mathrm{H}_{6} \mathrm{~F}_{3} \mathrm{O}_{2}\right]^{-:}: 191.0325$, found $191.0481\left([\mathrm{M}-\mathrm{H}]^{-}\right)$. 
(2-(benzyloxy)-4-(trifluoromethyl)phenyl)methanol (3-3)<smiles>OCc1ccc(C(F)(F)F)cc1OCc1ccccc1</smiles>

The preparation method of 3-3 was similar to 2-3.

The product yield: 2-(hydroxymethyl)-5-(trifluoromethyl)phenol(3-3, $185.3 \mathrm{mg}, 42.08 \%$,). m.p: 83.1-85.3 ${ }^{\circ} \mathrm{C}$.

TLC (PE:EA 3:1): $\mathbf{R}_{\mathbf{f}}=0.5$

${ }^{1}$ H NMR (400 MHz, Methanol- $\left.d_{4}\right): \delta 7.60(\mathrm{~d}, J=7.8 \mathrm{~Hz}, 1 \mathrm{H}), 7.46(\mathrm{~d}, J=7.4 \mathrm{~Hz}, 2 \mathrm{H}), 7.38$ (t, $J$ $=7.4 \mathrm{~Hz}, 2 \mathrm{H}), 7.32(\mathrm{~d}, J=7.3 \mathrm{~Hz}, 1 \mathrm{H}), 7.28-7.21(\mathrm{~m}, 2 \mathrm{H}), 5.17(\mathrm{~s}, 2 \mathrm{H}), 4.73(\mathrm{~s}, 2 \mathrm{H})$.

${ }^{13}$ C NMR (101 MHz, MeOD-d $\left.)_{4}\right): \delta 155.74$ (s, 1C), 136.65 (s, 1C), 134.51 (s, 1C), 129.80 (q, $J=$ $32.1 \mathrm{~Hz}, 1 \mathrm{C}$ ), 128.25 (d, $J=10.5 \mathrm{~Hz}, 2 \mathrm{C}$ ), 127.69 (s, 1C), 127.33 (s, 1C), 127.12 (s, 2C), 124.26 (dd, $J=542.4,271.2 \mathrm{~Hz}, 1 \mathrm{C}), 117.01$ (q, $J=4.1 \mathrm{~Hz}, 1 \mathrm{C}), 107.77$ (q, $J=3.7 \mathrm{~Hz}, 1 \mathrm{C}), 69.90$ (s, 1C), 58.43 (s, 1C).

ESI-HRMS: $m / z$ calcd. for $\left[\mathrm{C}_{15} \mathrm{H}_{17} \mathrm{~F}_{3} \mathrm{NO}_{2}\right]^{+}: 300.1206$, found $191.0483\left(\left[\mathrm{M}+\mathrm{NH}_{4}\right]^{+}\right)$.

2-(benzyloxy)-1-(bromomethyl)-4-(trifluoromethyl)benzene (3-4)<smiles>FC(F)(F)c1ccc(CBr)c(OCc2ccccc2)c1</smiles>

The preparation method of 3-4 was similar to 2-4.

The product yield: 2-(benzyloxy)-1-(bromomethyl)-4-(trifluoromethyl)benzene(3-4, $134.5 \mathrm{mg}$, $73.54 \%)$.

m.p: $68.8-73.9^{\circ} \mathrm{C}$.

TLC (PE:EA 3:1): $\mathbf{R}_{\mathbf{f}}=0.95$

${ }^{1}$ H NMR (400 MHz, Methanol-d $\left.d_{4}\right): \delta 7.54(\mathrm{dd}, J=7.4,4.8 \mathrm{~Hz}, 3 \mathrm{H}), 7.39$ (t, $\left.J=7.4 \mathrm{~Hz}, 2 \mathrm{H}\right), 7.33$ $(\mathrm{t}, J=7.3 \mathrm{~Hz}, 1 \mathrm{H}), 7.28(\mathrm{~s}, 1 \mathrm{H}), 7.24(\mathrm{t}, J=9.7 \mathrm{~Hz}, 1 \mathrm{H})), 5.24(\mathrm{~s}, 2 \mathrm{H}), 4.63(\mathrm{~s}, 2 \mathrm{H})$.

${ }^{13}$ C NMR (101 MHz, MeOD-d 4 ): $\delta 156.72$ (s, 1C), 136.39 (s, 1C), 131.70 (s, 1C), 131.08 (s, 1C), 130.95 (s, 1C), 128.21 (s, 2C), 127.69 (s, 1C), 127.38 (dd, J = 504.5, 376.0 Hz, 1C), 127.11 (s, 2C), 117.14 (q, $J=4.0 \mathrm{~Hz}, 1 \mathrm{C}), 108.77$ (q, $J=3.9 \mathrm{~Hz}, 1 \mathrm{C}), 70.14$ (s, 1C), 26.43 (s, 1C). 
(E)-1-(2-(benzyloxy)-4-(trifluoromethyl)benzyl)-3-((2,6-difluorophenyl)diazenyl)-1H-pyrazole (3-5)

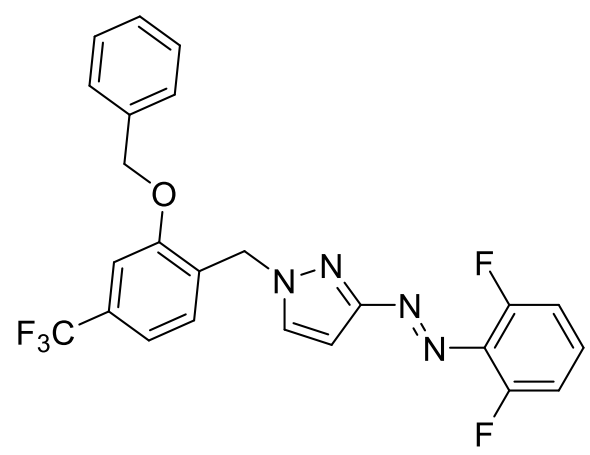

3-5

The preparation method of 3-5 was similar to piCRAC-1.

The product yield: (E)-1-(2-(benzyloxy)-4-(trifluoromethyl)benzyl)-3-((2,6-difluorophenyl)diazenyl)-1H-pyrazole $(3-5,59 \mathrm{mg}, 35.80 \%)$.

TLC (PE:EA 10:1): $\mathbf{R}_{\mathbf{f}}=0.4$

ESI-HRMS: $m / z$ calcd. for $\left[\mathrm{C}_{24} \mathrm{H}_{18} \mathrm{~F}_{5} \mathrm{~N}_{4} \mathrm{O}_{2}\right]^{+}: 473.1395$, found $473.1371\left([\mathrm{M}+\mathrm{H}]^{+}\right) . m / z$ calcd. for $\left[\mathrm{C}_{24} \mathrm{H}_{17} \mathrm{~F}_{5} \mathrm{~N}_{4} \mathrm{ONa}\right]^{+}$: 495.1215 , found $473.1371\left([\mathrm{M}+\mathrm{H}]^{+}\right) . \mathrm{m} / z$ calcd. for $\left[\mathrm{C}_{24} \mathrm{H}_{17} \mathrm{~F}_{5} \mathrm{~N}_{4} \mathrm{OK}\right]^{+}$: 511.0954, found $511.0924\left([\mathrm{M}+\mathrm{K}]^{+}\right)$.

(E)-2-((3-((2,6-difluorophenyl)diazenyl)-1H-pyrazol-1-yl)methyl)-5-(trifluoromethyl)phenol (piCRAC-3)<smiles>Oc1cc(C(F)(F)F)ccc1Cn1ccc(N=Nc2c(F)cccc2F)n1</smiles>

PICRAC-3

The preparation method of piCRAC-3 was similar to piCRAC-2.

It was to yield (E)-2-((3-((2,6-difluorophenyl)diazenyl)-1 H-pyrazol-1-yl)methyl)-5-(trifluoromethyl)phenol (piCRAC-3, $30 \mathrm{mg}, 75.38 \%$, Purity of trans $96.05 \%$, Sig = $290 \mathrm{~nm}, 4 \mathrm{~nm}$ ). m.p: $191.6-195.7^{\circ} \mathrm{C}$.

TLC (PE:EA 3:1): $\mathbf{R}_{\mathbf{f}}=0.4$.

${ }^{1} \mathbf{H}$ NMR (400 MHz, $\left.\mathbf{C D C l}_{3}\right): \delta 9.39(\mathrm{~s}, 1 \mathrm{H}), 7.56(\mathrm{~d}, J=2.5 \mathrm{~Hz}, 2 \mathrm{H}), 7.35(\mathrm{dt}, J=20.1,7.1 \mathrm{~Hz}$, 2H), 7.28 (broad, 2H), $7.16(\mathrm{~d}, J=7.7 \mathrm{~Hz}, 1 \mathrm{H}), 7.05(\mathrm{t}, J=8.7 \mathrm{~Hz}, 2 \mathrm{H}), 6.65(\mathrm{~d}, J=2.5 \mathrm{~Hz}, 1 \mathrm{H})$, $5.39(\mathrm{~s}, 2 \mathrm{H})$.

${ }^{13}$ C NMR (101 MHz, MeOD-d $\left.d_{4}\right): \delta 164.20$ (s, 1C), 156.91 (d, $\left.J=4.2 \mathrm{~Hz}, 1 \mathrm{C}\right), 155.97$ (s, 1C), 154.34 (d, $J=4.2 \mathrm{~Hz}, 1 \mathrm{C}$ ), 132.84 (s, 1C), 131.49 (s, 1C), 131.17 (s, 1C), 130.97 (dd, $J=23.7$, $13.4 \mathrm{~Hz}, 1 \mathrm{C}), 130.00$ (s, 2C), 125.71 (dd, $J=517.9,245.4 \mathrm{~Hz}, 1 \mathrm{C}), 115.49$ (s, 1C), $112.44-112.33$ (m, 1C), $112.22-112.13$ (m, 1C), 111.42 (s, 1C), 94.43 (s, 1C), 51.02 (s, 1C). 
ESI-HRMS: $m / z$ calcd. for $\left[\mathrm{C}_{17} \mathrm{H}_{12} \mathrm{~F}_{5} \mathrm{~N}_{4} \mathrm{O}\right]^{+}: 383.0926$, found $383.0932\left([\mathrm{M}+\mathrm{H}]^{+}\right), m / z$ calcd. for $\left[\mathrm{C}_{17} \mathrm{H}_{11} \mathrm{~F}_{5} \mathrm{~N}_{4} \mathrm{ONa}\right]^{+}:$405.0745, found $405.0748\left([\mathrm{M}+\mathrm{Na}]^{+}\right)$.

HPLC: mobile phase acetonitrile: $\mathrm{H}_{2} \mathrm{O}(70: 30)$, flow velocity $(1.0 \mathrm{~mL} / \mathrm{min}) . \mathrm{Sig}=290 \mathrm{~nm}, 4 \mathrm{~nm}$, purity of trans $96.05 \%$.

2-phenoxybenzaldehyde (4-3)<smiles>O=Cc1ccccc1Oc1ccccc1</smiles>

4-3

Phenol (4-1, 1 g, 10.63 mmol, 1.0 equiv), 2-fluorobenzaldehyde (4-2, 1.32 g, 10.63 mmol, 1.0 equiv) and copper(I) iodide ( $0.1 \mathrm{~g}, 10 \%)$ were added to the solution $(10 \mathrm{~mL}$ DMF). The mixture was heated at $120{ }^{\circ} \mathrm{C}$ for $12 \mathrm{~h}$. The mixture was extracted using EtOAc: $\mathrm{H}_{2} \mathrm{O}(50 \mathrm{~mL}: 50 \mathrm{~mL})$. The EA phase was washed with $\mathrm{H}_{2} \mathrm{O}(10 \mathrm{mLx} 3)$, and then washed with $\mathrm{NaCl}$ (aq. $10 \mathrm{~mL}$ ). The product was purified via column chromatography (PE) to yield white oil (4-3, $1.65 \mathrm{~g}, 78.20 \%$ ).

TLC (PE): $\mathbf{R}_{\mathbf{f}}=0.3$.

${ }^{1}$ H NMR (400 MHz, MeOD-d $)$ : $\delta 10.44(\mathrm{~s}, 1 \mathrm{H}), 7.89(\mathrm{~d}, J=7.8 \mathrm{~Hz}, 1 \mathrm{H}), 7.58(\mathrm{t}, J=7.8 \mathrm{~Hz}, 1 \mathrm{H})$, $7.42(\mathrm{t}, J=7.9 \mathrm{~Hz}, 2 \mathrm{H}), 7.35-7.29(\mathrm{~m}, 1 \mathrm{H}), 7.26-7.18(\mathrm{~m}, 2 \mathrm{H}), 7.08$ (d, J=7.9 Hz, 2H), $6.94-$ $6.88(\mathrm{~m}, 1 \mathrm{H})$.

${ }^{13}$ C NMR (101 MHz, MeOD-d d $_{4}: \delta 189.15$ (1C), 135.85 (1C), 129.89 (2C), 127.93 (1C), 124.12 (1C), 123.15 (1C), 119.05 (2C), 118.32 (1C), 117.78 (1C).

ESI-HRMS: $m / z$ calcd. for $\left[\mathrm{C}_{13} \mathrm{H}_{11} \mathrm{O}_{2}\right]^{+}: 199.0754$, found $199.0702\left([\mathrm{M}+\mathrm{H}]^{+}\right)$.

\section{(2-phenoxyphenyl)methanol (4-4)}<smiles>OCc1ccccc1Oc1ccccc1</smiles>

2-phenoxybenzaldehyde (4-3, $5.04 \mathrm{mmol}, 1.0$ equiv) was added to $10 \mathrm{~mL} \mathrm{MeOH}$, and sodium borohydride $(0.29 \mathrm{~g}, 7.57 \mathrm{mmol}, 1.5$ equiv) was added to the solution for $30 \mathrm{~min}$. The mixture was stirred for another $2 \mathrm{~h}$. The mixture was extracted with EA $(50 \mathrm{~mL})$. The aqueous phase was extracted with EA (10 mLx2), and the combined organic phase was dried by $\mathrm{Na}_{2} \mathrm{SO}_{4}$, (2-phenoxyphenyl)methanol was yielded as white oil (0.76 g, $75.25 \%)$.

TLC (PE:EA 10:1): $\mathbf{R}_{\mathbf{f}}=0.5$.

${ }^{1}$ H NMR (400 MHz, MeOD- $\left.d_{4}\right): \delta 7.53(\mathrm{~d}, J=7.4 \mathrm{~Hz}, 1 \mathrm{H}), 7.31(\mathrm{t}, J=7.9 \mathrm{~Hz}, 2 \mathrm{H}), 7.24(\mathrm{t}, J=$ $7.2 \mathrm{~Hz}, 1 \mathrm{H}), 7.15$ (t, $J=7.4 \mathrm{~Hz}, 1 \mathrm{H}), 7.06$ (t, $J=7.4 \mathrm{~Hz}, 1 \mathrm{H}), 6.91(\mathrm{~d}, J=7.9 \mathrm{~Hz}, 2 \mathrm{H}), 6.82$ (d, $J$ 
$=8.0 \mathrm{~Hz}, 1 \mathrm{H}), 4.65(\mathrm{~s}, 2 \mathrm{H})$.

${ }^{13}$ C NMR (101 MHz, MeOD-d $\left.d_{4}\right): \delta 157.67$ (1C), 153.90 (1C), 132.60 (1C), 129.45 (2C), 128.40 (1C), 128.25 (1C), 123.55 (1C), 122.62 (1C), 118.67 (1C), 117.56 (2C), 58.59 (1C).

ESI-HRMS: $\mathrm{m} / \mathrm{z}$ calcd. for $\left[\mathrm{C}_{13} \mathrm{H}_{11} \mathrm{O}\right]^{+}: 183.0804$, found $183.0778\left([\mathrm{M}-\mathrm{OH}]^{+}\right) \cdot \mathrm{m} / z$ calcd. for $\left[\mathrm{C}_{13} \mathrm{H}_{12} \mathrm{O}_{2}\right]^{+}:$200.0837, found $200.1030\left([\mathrm{M}]^{+}\right) . \mathrm{m} / z$ calcd. for $\left[\mathrm{C}_{13} \mathrm{H}_{12} \mathrm{O}_{2} \mathrm{Na}\right]^{+}: 223.0730$, found $223.0694\left([\mathrm{M}+\mathrm{Na}]^{+}\right)$.

1-(bromomethyl)-2-phenoxybenzene (4-5)<smiles>BrCc1ccccc1Oc1ccccc1</smiles>

The yield of product: 1-(bromomethyl)-2-phenoxybenzene (4-5, $506 \mathrm{mg}, 77.02 \%)$.

TLC (PE:EA 10:1): $\mathbf{R}_{\mathbf{f}}=0.8$

${ }^{1}$ H NMR (400 MHz, MeOD- $\left.\boldsymbol{d}_{4}\right): \delta 7.49-7.42(\mathrm{~m}, 1 \mathrm{H}), 7.38-7.30(\mathrm{~m}, 2 \mathrm{H}), 7.24(\mathrm{td}, J=8.2,1.6$ $\mathrm{Hz}, 1 \mathrm{H}), 7.16-7.05(\mathrm{~m}, 2 \mathrm{H}), 6.98(\mathrm{~d}, J=7.8 \mathrm{~Hz}, 2 \mathrm{H}), 6.78$ (d, $J=8.2 \mathrm{~Hz}, 1 \mathrm{H}), 4.59$ (s, 2H).

${ }^{13}$ C NMR (101 MHz, MeOD-d 4 ): $\delta 157.11$ (1C), 155.37 (1C), 131.15 (1C), 129.90 (1C), 129.58 (2C), 129.49 (1C), 123.39 (1C), 123.30 (1C), 118.52 (2C), 118.31 (1C), 27.18 (1C).

(E)-3-((2,6-difluorophenyl)diazenyl)-1-(2-phenoxybenzyl)-1H-pyrazole (piCRAC-4)<smiles>[Z6]C(=O)OCc1ccn(Cc2ccccc2Oc2ccccc2)n1</smiles>

The preparation method of piCRAC-4 was similar to piCRAC-1.

It was to yield (E)-3-((2,6-difluorophenyl)diazenyl)-1-(2-phenoxybenzyl)-1H-pyrazole(piCRAC-4, $156 \mathrm{mg}, 79.06 \%$, Purity of trans $97.90 \%$, Sig = $290 \mathrm{~nm}, 4 \mathrm{~nm}$ ).

m.p: $81.4-82.9^{\circ} \mathrm{C}$.

TLC (PE:EA 10:1): $\mathbf{R}_{\mathbf{f}}=0.5$

${ }^{1}$ H NMR (400 MHz, MeOD-d $\left.\boldsymbol{d}_{4}\right): \delta 7.67(\mathrm{~d}, J=2.5 \mathrm{~Hz}, 1 \mathrm{H}), 7.48-7.25(\mathrm{~m}, 5 \mathrm{H}), 7.17-7.05$ (m, 4H), $6.93(\mathrm{~d}, J=8.1 \mathrm{~Hz}, 2 \mathrm{H}), 6.85(\mathrm{~d}, J=8.2 \mathrm{~Hz}, 1 \mathrm{H}), 6.55(\mathrm{~d}, J=2.5 \mathrm{~Hz}, 1 \mathrm{H}), 5.50(\mathrm{~s}, 2 \mathrm{H})$.

${ }^{13}$ C NMR (101 MHz, MeOD-d $)$ ): $\delta 164.20(\mathrm{~s}), 156.88$ (d, $\left.J=3.6 \mathrm{~Hz}\right), 155.10(\mathrm{~s}), 154.31$ (d, $J=$ $4.2 \mathrm{~Hz}), 132.66(\mathrm{~s}), 130.87$ (t, $J=10.4 \mathrm{~Hz}), 130.35(\mathrm{~s}), 129.82(\mathrm{~s}), 129.61$ (s, 2C), 126.79 (s), $123.56(\mathrm{~s}), 123.33$ (s), 118.27 (s, 2C), 118.24 (s), 112.45 - $112.29(\mathrm{~m}), 112.24-112.09$ (m), 94.38 (s), $51.40(\mathrm{~s})$. 
${ }^{13}$ C NMR (101 MHz, MeOD-d $\left.d_{4}\right): \delta 164.20$ (s, 1C), 156.88 (d, $\left.J=3.6 \mathrm{~Hz}, 1 \mathrm{C}\right), 156.87$ (s, 1C), $155.10(\mathrm{~s}, 1 \mathrm{C}), 154.31$ (d, $J=4.2 \mathrm{~Hz}, 1 \mathrm{C}), 132.66$ (s, 1C), 130.99 (t, $J=5.1 \mathrm{~Hz}, 1 \mathrm{C}), 130.87$ (t, $J$ $=10.4 \mathrm{~Hz}, 1 \mathrm{C}), 130.35(\mathrm{~s}, 1 \mathrm{C}), 129.82(\mathrm{~s}, 1 \mathrm{C}), 129.61(\mathrm{~s}, 2 \mathrm{C}), 126.79(\mathrm{~s}, 1 \mathrm{C}), 123.56(\mathrm{~s}, 1 \mathrm{C})$, 123.33 (s, 1C), 118.27 (s, 2C), 118.24 (s, 1C), 112.45 - 112.29 (m, 1C), $112.24-112.09$ (m, 1C), 94.38 (s, 1C), $51.40(\mathrm{~s}, 1 \mathrm{C})$.

ESI-HRMS: $m / z$ calcd. for $\left[\mathrm{C}_{22} \mathrm{H}_{17} \mathrm{~F}_{2} \mathrm{~N}_{4} \mathrm{O}\right]^{+}: 391.1365$, found $391.1374\left([\mathrm{M}+\mathrm{H}]^{+}\right), m / z$ calcd. for $\left[\mathrm{C}_{22} \mathrm{H}_{16} \mathrm{~F}_{2} \mathrm{~N}_{4} \mathrm{ONa}\right]^{+}$: 413.1184, found $413.1090\left([\mathrm{M}+\mathrm{Na}]^{+}\right)$.

HPLC: mobile phase acetonitrile: $\mathrm{H}_{2} \mathrm{O}(75: 25)$, flow velocity $(1.0 \mathrm{~mL} / \mathrm{min}) . \mathrm{Sig}=290 \mathrm{~nm}, 4 \mathrm{~nm}$, purity of trans $97.90 \%$.

\section{2,5-dimethoxy-4'-nitro-1,1'-biphenyl (5-3)}<smiles>COc1ccc(OC)c(-c2ccc([N+](=O)[O-])cc2)c1</smiles>

2-bromo-1,4-dimethoxybenzene (5-1, $1 \mathrm{~g}, 4.63 \mathrm{mmol}, 1.0$ equiv), (4-nitrophenyl)boronic acid(5-2, $1.16 \mathrm{~g}, 6.95 \mathrm{mmol}, 1.5$ equiv), $\mathrm{K}_{2} \mathrm{CO}_{3}\left(1.28 \mathrm{~g}\right.$, $9.26 \mathrm{mmol}, 2.0$ equiv) and $\mathrm{PdCl}_{2}\left(\mathrm{PPh}_{3}\right)_{2}$ were added to toluene $/ \mathrm{EtOH} / \mathrm{H} 2 \mathrm{O}(20 \mathrm{~mL} / 40 \mathrm{~mL} / 10 \mathrm{~mL})$. The reaction was heated at $80{ }^{\circ} \mathrm{C}$ and kept for $8 \mathrm{~h}$ in $\mathrm{N}_{2}$. After that, the solvent was removed in vacuo. EA $(100 \mathrm{~mL})$ were used to extract the product. The aqueous layer was extracted with EA $(20 \mathrm{mLx} 2)$. The combined organic layer was washed with saturated brine $(20 \mathrm{~mL}) \mathbf{5 - 3}$ appeared as yellow solid $(1.15 \mathrm{~g}, 95.83 \%$, purity of trans $97.26 \%$, sig $=290 \mathrm{~nm}, 4 \mathrm{~nm})$.

m.p: $111.9-115.0^{\circ} \mathrm{C}$.

TLC (PE:EA 3:1): $\mathbf{R}_{\mathbf{f}}=0.5$

${ }^{1}$ H NMR (400 MHz, MeOD-d $\left.d_{4}\right): \delta 8.25(\mathrm{~d}, J=8.9 \mathrm{~Hz}, 2 \mathrm{H}), 7.74(\mathrm{~d}, J=8.9 \mathrm{~Hz}, 2 \mathrm{H}), 7.05(\mathrm{~d}, J=$ $8.9 \mathrm{~Hz}, 1 \mathrm{H}), 6.97(\mathrm{dd}, J=8.9,3.1 \mathrm{~Hz}, 1 \mathrm{H}), 6.93(\mathrm{~d}, J=3.0 \mathrm{~Hz}, 1 \mathrm{H}), 3.79(\mathrm{~s}, 3 \mathrm{H}), 3.76(\mathrm{~s}, 3 \mathrm{H})$.

${ }^{13}$ C NMR (101 MHz, DMSO-d $\left.{ }_{6}\right): \delta 153.22$ (1C), 149.88 (1C), 145.86 (1C), 144.62 (1C), 129.35 (2C), 128.14 (1C), 121.82 (2C), 115.26 (1C), 113.54 (1C), 111.95 (1C), 54.45 (1C), 53.99 (1C).

ESI-HRMS: $m / z$ calcd. for $\left[\mathrm{C}_{14} \mathrm{H}_{14} \mathrm{NO}_{4}\right]^{+}: 260.0914$, found $260.0869\left([\mathrm{M}+\mathrm{H}]^{+}\right), \mathrm{m} / z$ calcd. for $\left[\mathrm{C}_{14} \mathrm{H}_{13} \mathrm{NO}_{4} \mathrm{Na}\right]^{+}:$282.0737, found $282.0685\left([\mathrm{M}+\mathrm{Na}]^{+}\right)$.

(E)-4-((2',5'-dimethoxy-[1,1'-biphenyl]-4-yl)diazenyl)pyridine (piCRAC-5)<smiles>COc1ccc(OC)c(-c2ccc(/N=N/c3ccncc3)cc2)c1</smiles>

piCRAC-5

2,5-dimethoxy-4'-nitro-1,1'-biphenyl (5-3, 100 mg, 0.39 mmol, 1.0 equiv), pyridin-4-amine (5- 
4, $43.6 \mathrm{mg}, 1.2$ equiv), sodium hydroxide (61.1 $\mathrm{mg}, 1.53 \mathrm{mmol}, 4.0$ equiv), potassium carbonate (53.3 mg, $0.39 \mathrm{mmol}, 1.0$ equiv) and tris(2-(2-methoxyethoxy)ethyl)amine (12.5 mg, $0.04 \mathrm{mmol}$, $1.0 \%)$ were added to xylene $(10 \mathrm{~mL})$. The reaction was heated at $140{ }^{\circ} \mathrm{C}$ for $12 \mathrm{~h}$. The mixture was purified via column chromatography directly. The yellow solid was obtained as piCRAC-5 (43.5 mg, 35.31\%).

m.p: $126.3-128.3^{\circ} \mathrm{C}$.

TLC (PE:EA 3:1): $\mathbf{R}_{\mathbf{f}}=0.5$

${ }^{1}$ H NMR (400 MHz, MeOD-d $\left.\boldsymbol{d}_{4}\right): \delta 8.77(\mathrm{~d}, J=4.3 \mathrm{~Hz}, 2 \mathrm{H}), 8.02(\mathrm{~d}, J=7.6 \mathrm{~Hz}, 2 \mathrm{H}), 7.85$ (d, $J=$ $4.4 \mathrm{~Hz}, 2 \mathrm{H}), 7.74$ (d, $J=7.6 \mathrm{~Hz}, 2 \mathrm{H}), 7.05$ (d, $J=8.3 \mathrm{~Hz}, 1 \mathrm{H}), 6.96$ (s, 2H), 3.80 (s, 3H), 3.78 (s, $3 \mathrm{H})$.

${ }^{13} \mathrm{C}$ NMR (101 MHz, MeOD): $\delta 154.03,151.10,150.87,150.55,143.48,130.23$ (4C), 122.77, $116.45,116.05,113.79,112.82,55.34,54.79$.

${ }^{13}$ C NMR (101 MHz, MeOD-d 4 ) 154.03, 151.10, 150.87, 150.55, 143.48, 130.23, 122.77, 116.45, $116.05,113.79,112.82,55.34,54.79$.

${ }^{13}$ C NMR (101 MHz, MeOD-d4) $\delta 154.03$ (1C), 151.10 (1C), 150.87 (1C), 150.55 (2C), 143.48 (1C), 130.23 (4C), 122.77 (2C), 116.45 (2C), 116.05 (1C), 113.79 (1C), 112.82 (1C), 55.34 (1C), $54.79(1 \mathrm{C})$.

ESI-HRMS: $m / z$ calcd. for $\left[\mathrm{C}_{19} \mathrm{H}_{18} \mathrm{~N}_{3} \mathrm{O}_{2}\right]^{+}: 320.1394$, found $320.1396\left([\mathrm{M}+\mathrm{H}]^{+}\right)$.

HPLC: mobile phase acetonitrile: $\mathrm{H}_{2} \mathrm{O}$ (70: 30), flow velocity $(1.0 \mathrm{~mL} / \mathrm{min})$. $\mathrm{Sig}=290 \mathrm{~nm}, 4 \mathrm{~nm}$, purity of trans $97.26 \%$.

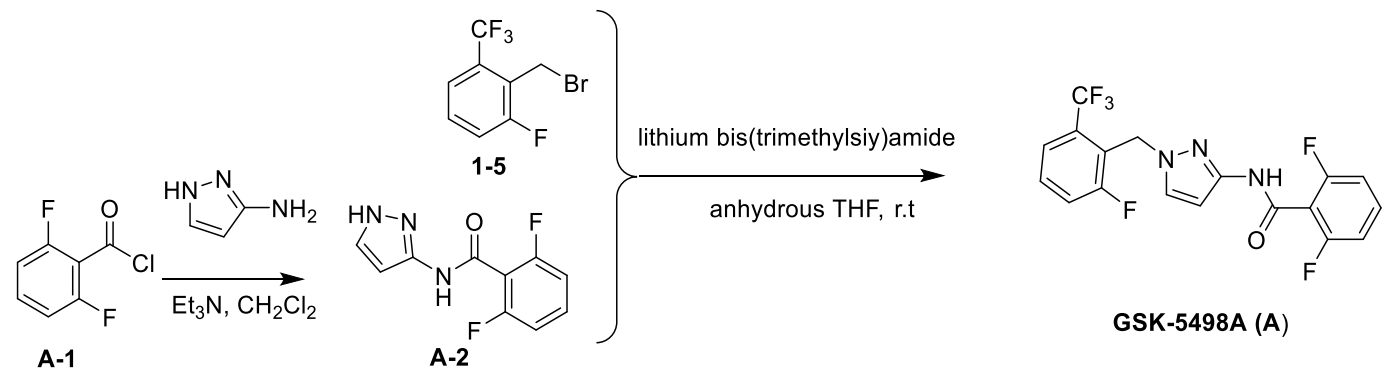

\section{Scheme S6 | Synthesis of GSK-5498A (Compound A)}

\section{2,6-difluoro-N-(1H-pyrazol-3-yl)benzamide (A-2)}<smiles>O=C(Nc1cc[nH]n1)c1c(F)cccc1F</smiles>

A-2

1H-pyrazol-3-amine (1-3, $2.5 \mathrm{~g}, 30.10 \mathrm{mmol}, 1.0$ equiv) and triethylamine (9.12 g, $90.31 \mathrm{mmol}$, 3.0 equiv) were dissolved in $10 \mathrm{~mL}$ acetonitrile. 2,6-difluorobenzoyl chloride (A-1, $5.30 \mathrm{~g} 30.10$ mmol, 1.0 equiv) was drop wised into the mixture for $30 \mathrm{~min}$ at $0{ }^{\circ} \mathrm{C}$. The reaction was transferred to room temperature and stirred for $12 \mathrm{~h}$. Then, the mixture was purified via column 
chromatography (PE: EA 1:2). The white solid was obtained as A-2 (5.42 g, 80.77 \%).

m.p: $203.8-206.1^{\circ} \mathrm{C}$.

TLC (PE:EA1:1): $\mathbf{R}_{\mathbf{f}}=0.3$

${ }^{1}$ H NMR (400 MHz, MeOD-d $\left.\boldsymbol{d}_{4}\right): \delta 7.60(\mathrm{~d}, J=2.1 \mathrm{~Hz}, 1 \mathrm{H}), 7.57-7.46(\mathrm{~m}, 1 \mathrm{H}), 7.09$ (p, $J=2.6$ $\mathrm{Hz}, 2 \mathrm{H}), 6.69$ (broad, 1H).

${ }^{13}$ C NMR (101 MHz, MeOD- $\left.d_{4}\right): \delta 160.88$ (d, $\left.J=11.3 \mathrm{~Hz}, 1 \mathrm{C}\right), 159.15$ (s, 1C), 158.41 (d, $J=7.2$ $\mathrm{Hz}, 1 \mathrm{C}), 132.89-131.22$ (m, 1C), 128.99 (s, 1C), 114.58 (s, 1C), $111.65-111.52$ (m, 1C), 111.44 -111.30 (m, 1C), 96.72 (s, 1C).

ESI-HRMS: $m / z$ calcd. for $\left[\mathrm{C}_{10} \mathrm{H}_{8} \mathrm{~F}_{2} \mathrm{~N}_{3} \mathrm{O}\right]^{+}: 246.0449$ found $224.0584\left([\mathrm{M}+\mathrm{H}]^{+}\right) \cdot \mathrm{m} / z$ calcd. for $\left[\mathrm{C}_{10} \mathrm{H}_{7} \mathrm{~F}_{2} \mathrm{~N}_{3} \mathrm{ONa}\right]^{+}:$224.0630, found $246.0397\left([\mathrm{M}+\mathrm{Na}]^{+}\right)$.

2,6-difluoro-N-(1-(2-fluoro-6-(trifluoromethyl)benzyl)-1H-pyrazol-3-yl)benzamide (GSK5498A (A))

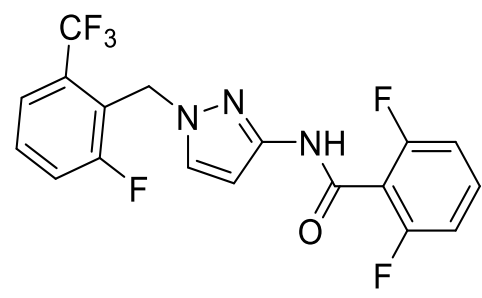

GSK-5498A (A)

The preparation method of GSK-5498A was similar to piCRAC-1.

2,6-difluoro-N-(1-(2-fluoro-6-(trifluoromethyl)benzyl)-1H-pyrazol-3-yl)benzamide (GSK5498A, 295mg, $86.14 \%$, Purity96.86 \%, Sig=275 nm, 4 nm).

m.p: $138.0-145.7^{\circ} \mathrm{C}$.

TLC (PE:EA 5:1): $\mathbf{R}_{\mathbf{f}}=0.2$

${ }^{1}$ H NMR (400 MHz, MeOD-d $\left.\boldsymbol{d}_{4}\right): \delta 7.69-7.57(\mathrm{~m}, 2 \mathrm{H}), 7.54-7.37(\mathrm{~m}, 3 \mathrm{H}), 7.05(\mathrm{t}, J=8.1 \mathrm{~Hz}$, $2 \mathrm{H}), 6.67(\mathrm{~d}, J=2.4 \mathrm{~Hz}, 1 \mathrm{H}), 5.45(\mathrm{~s}, 2 \mathrm{H})$.

${ }^{13}$ C NMR (101 MHz, MeOD-d 4 ): $\delta 163.76$ (s, 1C), 161.23 (s, 1C), 160.87 (d, $J=10.2 \mathrm{~Hz}, 1 \mathrm{C}$ ), 159.03 (s, 1C), 158.38 (d, $J=11.1 \mathrm{~Hz}, 1 \mathrm{C}), 146.53$ (s, 1C), 131.88 (t, $J=10.1 \mathrm{~Hz}, 1 \mathrm{C}), 131.05$ (s, 1C), 130.96 (s, 1C), 130.14 (s, 1C), $122.11-121.75$ (m, 1C), 119.84 (s, 1C), 119.62 (s, 1C), 114.48 (t, $J=21.7 \mathrm{~Hz}, 1 \mathrm{C}), 111.65-111.48$ (m, 1C), $111.42-111.23$ (m, 1C), 97.38 (s, 1C), 45.11 (s, $1 \mathrm{C)}$.

ESI-HRMS: $m / z$ calcd. for $\left[\mathrm{C}_{18} \mathrm{H}_{12} \mathrm{~F}_{6} \mathrm{~N}_{3} \mathrm{O}\right]^{+}: 400.0879$ found $400.0871\left([\mathrm{M}+\mathrm{H}]^{+}\right) . \mathrm{m} / z$ calcd. for $\left[\mathrm{C}_{18} \mathrm{H}_{11} \mathrm{~F}_{6} \mathrm{~N}_{3} \mathrm{ONa}\right]^{+}:$422.0699, found $422.0665\left([\mathrm{M}+\mathrm{Na}]^{+}\right)$.

HPLC: mobile phase acetonitrile: $\mathrm{H}_{2} \mathrm{O}$ (70:30), flow velocity $(1.0 \mathrm{~mL} / \mathrm{min}) . \mathrm{Sig}=275 \mathrm{~nm}, 4 \mathrm{~nm}$, purity of trans $96.86 \%$.

(E)-3-(phenyldiazenyl)-1H-pyrazole (6-3) 


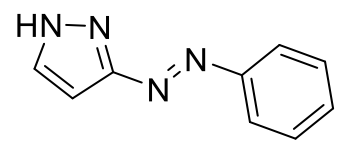

6-3

A yellow solid was obtained (326 mg, 52.44\%).

m.p: $109.7-113.3^{\circ} \mathrm{C}$.

TLC (PE:EA 3:1): $\mathbf{R}_{\mathbf{f}}=0.4$

${ }^{1}$ H NMR (400 MHz, DMSO-d $\left.\boldsymbol{d}_{6}\right) \delta 13.48(\mathrm{~s}, 1 \mathrm{H}), 7.88(\mathrm{~s}, 1 \mathrm{H}), 7.86(\mathrm{~d}, J=7.7 \mathrm{~Hz}, 2 \mathrm{H}), 7.69-7.51(\mathrm{~m}$, $3 \mathrm{H}), 6.57(\mathrm{~s}, 1 \mathrm{H})$.

${ }^{13}$ C NMR (101 MHz, DMSO-d $\left.\boldsymbol{d}_{\boldsymbol{6}}\right) \delta$ 164.37, 152.66, 131.68, 131.13, 129.93 (2C), $122.74(2 \mathrm{C}), 93.90$

ESI-HRMS: $m / z$ calcd. for $\left[\mathrm{C}_{9} \mathrm{H}_{19} \mathrm{~N}_{4}\right]^{+}: 173.0822$ found $173.0789\left([\mathrm{M}+\mathrm{H}]^{+}\right)$.

(E)-1-(2-fluoro-6-(trifluoromethyl)benzyl)-3-(phenyldiazenyl)-1H-pyrazole (piCRAC-6)

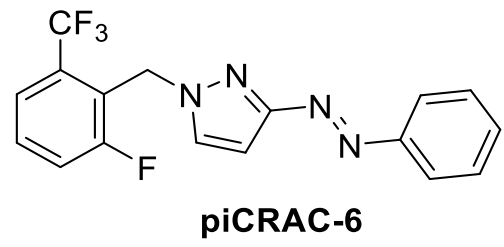

A yellow solid was obtained (13 mg, 6.43\%).

m.p: $74.0-76.1^{\circ} \mathrm{C}$.

TLC (PE:EA 10:1): $\mathbf{R}_{\mathbf{f}}=0.6$

${ }^{1} \mathrm{H}$ NMR (400 MHz, MeOD-d 4 ) $\delta 7.88(\mathrm{~d}, J=7.5 \mathrm{~Hz}, 2 \mathrm{H}), 7.67(\mathrm{~d}, J=15.6 \mathrm{~Hz}, 3 \mathrm{H}), 7.60-7.46(\mathrm{~m}, 4 \mathrm{H})$, $6.64(\mathrm{~s}, 1 \mathrm{H}), 5.67(\mathrm{~s}, 2 \mathrm{H})$.

${ }^{13}$ C NMR (101 MHz, MeOD- $\left.d_{4}\right) \delta 163.76(\mathrm{~d}, J=5.6 \mathrm{~Hz}), 161.30(\mathrm{~s}), 152.67$ (s), $131.84(\mathrm{~s}), 131.33$ (d, $J=$ $9.5 \mathrm{~Hz}$ ), 131.07 (s), 128.88 (s, 2C), $130.54-124.44$ (m), 122.35 (s, 2C), 122.11 (dt, $J=9.3,4.1 \mathrm{~Hz}$ ),

120.50 (d, $J=17.0 \mathrm{~Hz}), 119.89$ (d, $J=23.0 \mathrm{~Hz}), 116.98$ (s), 94.50 (s), 46.01 (s).

${ }^{19}$ F NMR (376 MHz, MeOD-d d $_{4} \delta-59.86$ (s 3F), -114.12 (d, $\left.J=8.7 \mathrm{~Hz}, 1 \mathrm{~F}\right)$

ESI-HRMS: $m / z$ calcd. for $\left[\mathrm{C}_{17} \mathrm{H}_{14} \mathrm{~F}_{4} \mathrm{~N}_{4}\right]^{+}: 349.1071$ found $349.1112\left([\mathrm{M}+\mathrm{H}]^{+}\right)$.

HPLC: mobile phase acetonitrile: $\mathrm{H}_{2} \mathrm{O}(75: 25)$, flow velocity $(1.0 \mathrm{~mL} / \mathrm{min}) . \mathrm{Sig}=302 \mathrm{~nm}, 4 \mathrm{~nm}$, purity of trans $94.39 \%$.

methyl 3,5-difluoro-4-nitrosobenzoate (7-2)<smiles>CC(=O)c1cc(F)c([N+](=O)[O-])c(F)c1</smiles>

7-2

The emerald solid was obtained (270 mg, 100\%).

m.p: $150.0-152.6^{\circ} \mathrm{C}$. 
$\operatorname{TLC}(\mathbf{P E}): \mathbf{R}_{\mathbf{f}}=0.6$

${ }^{1}$ H NMR (400 MHz, CDCl $) \delta 7.78(\mathrm{~d}, J=9.9 \mathrm{~Hz}, 2 \mathrm{H}), 4.00(\mathrm{~s}, 3 \mathrm{H})$.

${ }^{13}$ C NMR (101 MHz, CDCl $) \delta 163.45$ (s), $153.02(\mathrm{~d}, J=270.4 \mathrm{~Hz}, 2 \mathrm{C}), 137.12$ (s), $116.91-110.82(\mathrm{~m}$, 2C), 53.35 (s).

${ }^{19}$ F NMR (376 MHz, CDCl 3$) \delta-128.93$ (s, 2F).

methyl (E)-4-((1H-pyrazol-3-yl)diazenyl)-3,5-difluorobenzoate (7-3)<smiles>COC(=O)c1cc(F)c(N=Nc2cc[nH]n2)c(F)c1</smiles>

A yellow solid was obtained (35 mg, 22.98\%).

m.p: $186.5-189.3^{\circ} \mathrm{C}$.

TLC (PE:EA=3:1): $\mathbf{R}_{\mathbf{f}}=0.4$

${ }^{1}$ H NMR (400 MHz, MeOD-d $\left.)_{4}\right) \quad 7.95-7.52(\mathrm{~m}, 2 \mathrm{H}), 6.33(\mathrm{~d}, J=290.1 \mathrm{~Hz}, 1 \mathrm{H}), 3.97(\mathrm{~d}, J=9.1 \mathrm{~Hz}$, $3 \mathrm{H})$.

${ }^{13}$ C NMR (101 MHz, MeOD-d $\left.d_{4}\right) \delta 170.65$ (s), 149.43 (s), 130.33 (d, $J=48.4 \mathrm{~Hz}, 2 \mathrm{C}$ ), 113.34 (d, $J=20.1$ Hz, 2C), 99.82 (s), 93.47 (s), 51.92 (d, $J=10.9 \mathrm{~Hz})$.

${ }^{19} \mathrm{~F}$ NMR $(376 \mathrm{MHz}, \mathrm{MeOD}) \delta-123.04(\mathrm{dd}, J=496.7,8.4 \mathrm{~Hz}, 2 \mathrm{~F})$

ESI-HRMS: $m / z$ calcd. for $\left[\mathrm{C}_{11} \mathrm{H}_{9} \mathrm{~F}_{2} \mathrm{~N}_{4} \mathrm{O}_{2}\right]^{+}: 267.0688$ found $267.0613\left([\mathrm{M}+\mathrm{H}]^{+}\right)$.

methyl (E)-3,5-difluoro-4-((1-(2-fluoro-6-(trifluoromethyl)benzyl)-1H-pyrazol-3-yl)diazenyl)benzoate (piCRAC-7)

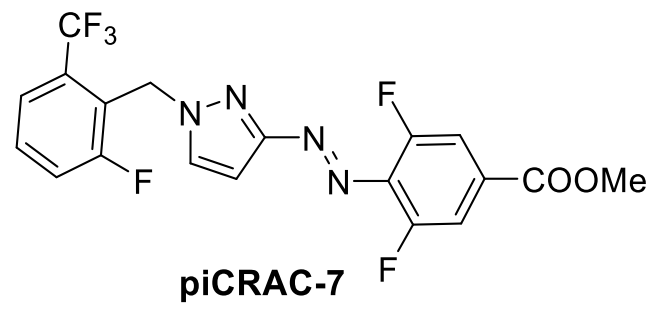

A yellow solid was obtained (9 mg, 18.01\%).

m.p: $151.2-152.8^{\circ} \mathrm{C}$.

TLC (PE:EA=3:1): $\mathbf{R}_{\mathbf{f}}=0.6$

${ }^{1} \mathrm{H}$ NMR (400 MHz, MeOD) $\delta 7.77$ (s, 1H), $7.75(\mathrm{~s}, 1 \mathrm{H}), 7.71(\mathrm{~s}, 3 \mathrm{H}), 7.57$ (dd, $\left.J=11.5,6.6 \mathrm{~Hz}, 1 \mathrm{H}\right), 6.66$ (s, 1H), $5.71(\mathrm{~s}, 2 \mathrm{H}), 3.97(\mathrm{~s}, 3 \mathrm{H})$.

${ }^{13} \mathrm{C}$ NMR (151 MHz, MeOD) $\delta 157.56(\mathrm{~d}, J=523.2 \mathrm{~Hz}), 150.05(\mathrm{~d}, J=256.9 \mathrm{~Hz}, 2 \mathrm{C}), 132.21(\mathrm{~d}, J=22.7$ $\mathrm{Hz}), 131.45$ (d, $J=9.6 \mathrm{~Hz}), 130.93$ (d, $J=9.4 \mathrm{~Hz}), 122.29-121.97$ (m), 120.86 (dd, $J=282.6,75.3 \mathrm{~Hz}$ ), 119.92 (d, $J=23.0 \mathrm{~Hz}), 119.35$ (d, $J=23.5 \mathrm{~Hz}), 113.29$ (dd, $J=21.5,4.5 \mathrm{~Hz}), 112.46$ (dd, $J=20.4,5.1 \mathrm{~Hz})$, $94.53(\mathrm{~s}), 51.88(\mathrm{~d}, J=29.6 \mathrm{~Hz})$.

${ }^{19} \mathrm{~F}$ NMR (376 MHz, MeOD) $\delta-59.91(\mathrm{~s}, 3 \mathrm{~F}),-114.12(\mathrm{~d}, J=11.0 \mathrm{~Hz}, 1 \mathrm{~F}),-122.19$ (d, $\left.J=9.6 \mathrm{~Hz}, 2 \mathrm{~F}\right)$.

ESI-HRMS: $m / z$ calcd. for $\left[\mathrm{C}_{19} \mathrm{H}_{13} \mathrm{~F}_{6} \mathrm{~N}_{4} \mathrm{O}_{2}\right]^{+}: 443.0937$ found $443.0945\left([\mathrm{M}+\mathrm{H}]^{+}\right), m / z$ calcd. for 
$\left[\mathrm{C}_{19} \mathrm{H}_{13} \mathrm{~F}_{6} \mathrm{~N}_{4} \mathrm{NaO}_{2}\right]^{+}: 465.0757$ found $465.0757\left([\mathrm{M}+\mathrm{Na}]^{+}\right)$

HPLC: mobile phase acetonitrile: $\mathrm{H}_{2} \mathrm{O}(75: 25)$, flow velocity $(1.0 \mathrm{~mL} / \mathrm{min}) . \mathrm{Sig}=302 \mathrm{~nm}, 4 \mathrm{~nm}$, purity of trans $93.34 \%$.

methyl 3,5-difluoro-4-nitrosobenzoate (8-2)<smiles>O=[N+]([O-])c1c(F)cc(F)cc1F</smiles>

8-2

A yellow solid was obtained $(0.98 \mathrm{~g}, 89.91 \%)$.

m.p: $98.8-99.8^{\circ} \mathrm{C}$.

TLC (PE:EA=10:1): $\mathbf{R}_{\mathbf{f}}=0.6$

${ }^{1} \mathrm{H}$ NMR (400 MHz, $\left.\mathbf{C D C l}_{3}\right) \delta 6.86(\mathrm{t}, J=9.1 \mathrm{~Hz}, 2 \mathrm{H})$.

${ }^{13}$ C NMR (101 MHz, CDCl $) \delta 188.56(\mathrm{~d}, J=516.0 \mathrm{~Hz}), 165.95(\mathrm{~d}, J=263.1 \mathrm{~Hz}, 2 \mathrm{C}), 155.32$ (ddd, $J=$ $272.3,16.1,6.1 \mathrm{~Hz}), 101.91-101.21(\mathrm{~m}, 2 \mathrm{C})$.

${ }^{19}$ F NMR (376 MHz, CDCl$) \delta-91.32--92.52(\mathrm{~m}, 1 \mathrm{~F}),-125.07(\mathrm{dd}, J=12.4,10.1 \mathrm{~Hz}, 2 \mathrm{~F})$.

(E)-3-((2,4,6-trifluorophenyl)diazenyl)-1H-pyrazole (8-3)<smiles>Fc1cc(F)c(N=Nc2cc[nH]n2)c(F)c1</smiles>

A yellow solid was obtained (250 $\mathrm{mg}, 18.12 \%)$.

m.p: $154.2-157.3^{\circ} \mathrm{C}$.

TLC (PE:EA=3:1): $\mathbf{R}_{\mathbf{f}}=0.4$

${ }^{1}$ H NMR (400 MHz, DMSO-d 6 ) $\delta 13.65$ (s, 1H), 7.92 (s, 1H), 7.45 (t, $J=9.9$ Hz, 2H), 6.55 (s, 1H).

${ }^{13}$ C NMR (101 MHz, DMSO-d 6 ) $\delta 164.93$ (s), 162.31 (dt, $J=250.7,15.6 \mathrm{~Hz}$ ), 155.98 (ddd, $J=258.5,16.0$, $7.1 \mathrm{~Hz}, 2 \mathrm{C}$ ), 131.51 (s), $132.61-127.67$ (m), 102.57 (td, $J=26.6,3.6 \mathrm{~Hz}, 2 \mathrm{C}), 93.83$ (s).

${ }^{19}$ F NMR (376 MHz, DMSO-d $) \delta-104.94$ (p, $\left.J=8.1 \mathrm{~Hz}, 1 \mathrm{~F}\right),-118.06--118.16$ (m, 2F).

ESI-HRMS: $m / z$ calcd. for $\left[\mathrm{C}_{9} \mathrm{H}_{6} \mathrm{~F}_{3} \mathrm{~N}_{4}\right]^{+}: 227.0539$ found $227.0612\left([\mathrm{M}+\mathrm{H}]^{+}\right)$.

(E)-1-(2-fluoro-6-(trifluoromethyl)benzyl)-3-((2,4,6-trifluorophenyl)diazenyl)-1H-pyrazole (piCRAC-8)

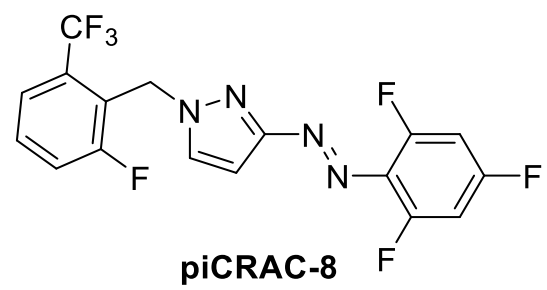

A yellow solid was obtained (54 mg, 34.5\%). 
m.p: $101.3-102.6^{\circ} \mathrm{C}$.

TLC (PE:EA=10:1): $\mathbf{R}_{\mathbf{f}}=0.4$

${ }^{1}$ H NMR (400 MHz, MeOD-d $\left.d_{4}\right) \delta 7.68(\mathrm{~d}, J=10.3 \mathrm{~Hz}, 3 \mathrm{H}), 7.55(\mathrm{dd}, J=10.3,5.2 \mathrm{~Hz}, 1 \mathrm{H}), 7.07$ (t, $J=9.5$ $\mathrm{Hz}, 2 \mathrm{H}), 6.61(\mathrm{~s}, 1 \mathrm{H}), 5.68(\mathrm{~s}, 2 \mathrm{H})$.

${ }^{13}$ C NMR (101 MHz, MeOD-d 4 ) $\delta 164.29$ (s), 162.71 (dd, $\left.J=252.4,15.1 \mathrm{~Hz}\right), 162.71$ (d, $J=132.7,117.4$ Hz), 156.41 (ddd, $J=260.4,15.1,6.7 \mathrm{~Hz}), 132.04$ (s), 131.39 (d, $J=9.5 \mathrm{~Hz}), 128.31$ - 127.94 (m), 130.97 122.95 (m), 122.23 (d, $J=4.1 \mathrm{~Hz}), 122.12$ (dt, $J=9.4,4.0 \mathrm{~Hz}$ ), 120.34 (d, $J=17.0 \mathrm{~Hz}$ ), 119.90 (d, $J=22.9$ $\mathrm{Hz}), 101.45$ - 100.75 (m, 2C), 94.26 (s), 46.08 (s).

${ }^{19}$ F NMR (376 MHz, MeOD-d $) \delta-59.90$ (s, 3F), -106.37 (p, $\left.J=8.1 \mathrm{~Hz}, 1 \mathrm{~F}\right),-114.11$ (d, $\left.J=9.0 \mathrm{~Hz}, 1 \mathrm{~F}\right),-$ $118.55(\mathrm{t}, J=8.5 \mathrm{~Hz}, 2 \mathrm{~F})$.

ESI-HRMS: $m / z$ calcd. for $\left[\mathrm{C}_{17} \mathrm{H}_{10} \mathrm{~F}_{7} \mathrm{~N}_{4}\right]^{+}: 403.0788$ found $403.0719\left([\mathrm{M}+\mathrm{H}]^{+}\right)$,

HPLC: mobile phase acetonitrile: $\mathrm{H}_{2} \mathrm{O}(75: 25)$, flow velocity $(1.0 \mathrm{~mL} / \mathrm{min}) . \mathrm{Sig}=302 \mathrm{~nm}, 4 \mathrm{~nm}$, purity of trans $99.31 \%$.

\section{5-chloro-1,3-difluoro-2-nitrosobenzene (9-2)}<smiles>O=[N+]([O-])c1c(F)cc(Cl)cc1F</smiles>

9-2

A dark yellow solid was obtained (1.05 g, 97.22\%).

m.p: $88.0-89.0^{\circ} \mathrm{C}$.

TLC (PE:EA=10:1): $\mathbf{R}_{\mathbf{f}}=0.6$

${ }^{1}$ H NMR (400 MHz, $\left.\mathbf{C D C l}_{3}\right) \delta 7.16(\mathrm{~d}, J=9.5 \mathrm{~Hz}, 2 \mathrm{H})$.

${ }^{13}$ C NMR (101 MHz, CDCl $) \delta 153.71(\mathrm{dd}, J=273.0,4.7 \mathrm{~Hz}, 2 \mathrm{C}), 145.52(\mathrm{t}, J=8.4 \mathrm{~Hz}), 142.70$ (t, $J=13.4$ $\mathrm{Hz}), 114.03$ (dd, $J=23.4,3.7 \mathrm{~Hz}, 2 \mathrm{C}$ ).

${ }^{19}$ F NMR (376 MHz, CDCl 3$) ~ \delta-128.15(\mathrm{~d}, J=9.4 \mathrm{~Hz}, 2 \mathrm{~F})$.

(E)-3-((4-chloro-2,6-difluorophenyl)diazenyl)-1H-pyrazole (9-3)

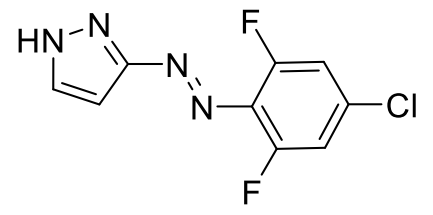

9-3

A yellow solid was obtained (56 mg, 40.99\%).

m.p: $178.9-179.6^{\circ} \mathrm{C}$.

TLC (PE:EA=3:1): $\mathbf{R}_{\mathbf{f}}=0.4$

${ }^{1}$ H NMR (400 MHz, DMSO-d 6 ) $\delta 13.70(\mathrm{~s}, 1 \mathrm{H}), 7.93$ (s, 1H), 7.62 (d, $\left.J=9.5 \mathrm{~Hz}, 2 \mathrm{H}\right), 6.56(\mathrm{~s}, 1 \mathrm{H})$. 
${ }^{13}$ C NMR (101 MHz, DMSO-d 6 ) $\delta 164.98(\mathrm{~s}), 155.29(\mathrm{dd}, J=259.5,5.9 \mathrm{~Hz}), 135.14(\mathrm{t}, J=13.4 \mathrm{~Hz})$, 131.60 (s), 129.99 (t, $J=10.6 \mathrm{~Hz}), 114.60$ (d, $J=27.9 \mathrm{~Hz}, 2 \mathrm{C}), 93.95$ (s).

${ }^{19}$ F NMR (376 MHz, DMSO-d 6 ) $\delta-119.87$ (d, $\left.J=9.5 \mathrm{~Hz}, 2 \mathrm{~F}\right)$.

ESI-HRMS: $m / z$ calcd. for $\left[\mathrm{C}_{9} \mathrm{H}_{6} \mathrm{ClF}_{2} \mathrm{~N}_{4}\right]^{+}: 243.0244$ found $243.0309\left([\mathrm{M}+\mathrm{H}]^{+}\right), \mathrm{m} / z$ calcd. for $\left[\mathrm{C}_{9} \mathrm{H}_{5} \mathrm{ClF}_{2} \mathrm{~N}_{4} \mathrm{Na}\right]^{+}: 265.0063$ found $265.0132\left([\mathrm{M}+\mathrm{Na}]^{+}\right)$.

(E)-3-((4-chloro-2,6-difluorophenyl)diazenyl)-1-(2-fluoro-6-(trifluoromethyl)benzyl)-1H-pyrazole (piCRAC-9)

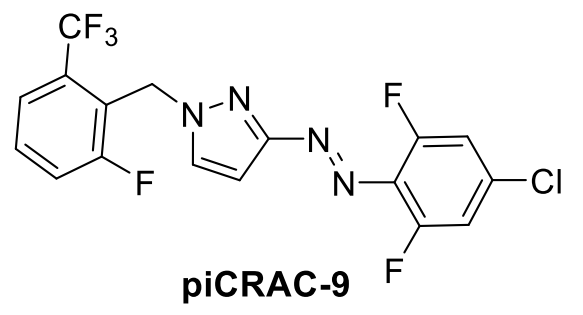

A yellow solid was obtained (12 mg, 13.91\%).

m.p: $97.4-98.3{ }^{\circ} \mathrm{C}$.

TLC (PE:EA=10:1): $\mathbf{R}_{\mathbf{f}}=0.5$

${ }^{1}$ H NMR (400 MHz, MeOD-d $\left.d_{4}\right) \delta 7.68(\mathrm{~d}, J=5.3 \mathrm{~Hz}, 3 \mathrm{H}), 7.55(\mathrm{dd}, J=10.1,4.9 \mathrm{~Hz}, 1 \mathrm{H}), 7.30$ (d, $J=9.7$ $\mathrm{Hz}, 2 \mathrm{H}), 6.61(\mathrm{~s}, 1 \mathrm{H}), 5.69$ (s, 2H).

${ }^{13}$ C NMR (101 MHz, MeOD-d 4 ) $\delta 164.32(\mathrm{~s}), 162.55$ (d, $\left.J=253.1 \mathrm{~Hz}\right), 155.58(\mathrm{dd}, J=261.7,5.6 \mathrm{~Hz}, 2 \mathrm{C})$, 135.72 (t, $J=13.2 \mathrm{~Hz}$ ), 132.12 (s), 131.41 (d, $J=9.5 \mathrm{~Hz}), 129.96-129.61(\mathrm{~m}), 126.43$ (dd, $J=549.8,279.0$ $\mathrm{Hz}), 122.22(\mathrm{~d}, J=2.6 \mathrm{~Hz}), 122.12(\mathrm{dt}, J=5.5,2.0 \mathrm{~Hz}), 120.30(\mathrm{~d}, J=15.8 \mathrm{~Hz}), 119.90(\mathrm{~d}, J=22.7 \mathrm{~Hz})$, 113.65 - 113.18 (m, 2C), 94.35 (s), 46.14 (s).

${ }^{19}$ F NMR (376 MHz, MeOD-d $\left.d_{4}\right) \delta-59.90$ (s, 3F), -114.10 (d, $\left.J=8.8 \mathrm{~Hz}, 1 \mathrm{~F}\right),-120.58$ (d, $J=9.4 \mathrm{~Hz}, 2 \mathrm{~F}$ ).

ESI-HRMS: $m / z$ calcd. for $\left[\mathrm{C}_{9} \mathrm{H}_{6} \mathrm{ClF}_{2} \mathrm{~N}_{4}\right]^{+}: 243.0244$ found $243.0309\left([\mathrm{M}+\mathrm{H}]^{+}\right), \mathrm{m} / z$ calcd. for $\left[\mathrm{C}_{9} \mathrm{H}_{6} \mathrm{ClF}_{2} \mathrm{~N}_{4}\right]^{+}: 421.0463$ found $421.0463\left([\mathrm{M}+2+\mathrm{H}]^{+}\right)$,

HPLC: mobile phase acetonitrile: $\mathrm{H}_{2} \mathrm{O}(75: 25)$, flow velocity $(1.0 \mathrm{~mL} / \mathrm{min}) . \mathrm{Sig}=302 \mathrm{~nm}, 4 \mathrm{~nm}$, purity of trans $97.39 \%$

\section{5-bromo-1,3-difluoro-2-nitrosobenzene (10-2)}<smiles>O=[N+]([O-])c1c(F)cc(Br)cc1F</smiles>

10-2

A white solid was obtained $(0.95 \mathrm{~g}, 88.79 \%)$.

Sublimation point: $110.1-113.9^{\circ} \mathrm{C}$. 
TLC (PE:EA=10:1): $\mathbf{R}_{\mathbf{f}}=0.6$

${ }^{1}$ H NMR (400 MHz, CDCl 3$) \delta 7.34(\mathrm{~d}, J=9.1 \mathrm{~Hz}, 2 \mathrm{H})$.

${ }^{13}$ C NMR (101 MHz, CDCl $) \delta 153.38(\mathrm{dd}, J=274.2,4.4 \mathrm{~Hz}), 145.57$ (t, $\left.J=8.3 \mathrm{~Hz}\right), 130.61$ (t, $J=12.3$ $\mathrm{Hz}), 116.99(\mathrm{dd}, J=23.2,3.6 \mathrm{~Hz}, 2 \mathrm{C})$.

${ }^{19}$ F NMR (376 MHz, CDCl$) ~ \delta-128.64(\mathrm{~d}, J=8.9 \mathrm{~Hz}, 2 \mathrm{~F})$.

(E)-3-((4-bromo-2,6-difluorophenyl)diazenyl)-1H-pyrazole (10-3)

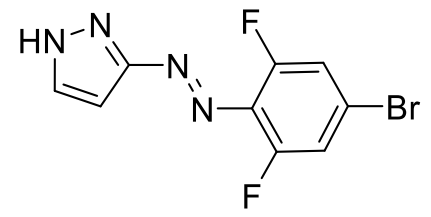

$10-3$

A yellow solid was obtained (65 mg, 50.27\%).

m.p: $171.9-173.3{ }^{\circ} \mathrm{C}$.

TLC (PE:EA=3:1): $\mathbf{R}_{\mathbf{f}}=0.5$

${ }^{1}$ H NMR (400 MHz, DMSO-d 6 ) $\delta 13.70(\mathrm{~s}, 1 \mathrm{H}), 7.93$ (s, 1H), 7.73 (d, $\left.J=8.9 \mathrm{~Hz}, 2 \mathrm{H}\right), 6.56(\mathrm{~s}, 1 \mathrm{H})$.

${ }^{13}$ C NMR (101 MHz, DMSO-d $\left.\boldsymbol{d}_{6}\right) 165.02$ (s), 155.24 (dd, $\left.J=260.3,5.9 \mathrm{~Hz}, 2 \mathrm{C}\right), 131.61$ (s), $130.24(\mathrm{t}, J=$ $10.5 \mathrm{~Hz}), 123.25-122.50$ (m), 117.37 (d, $J=24.9 \mathrm{~Hz}, 2 \mathrm{C}), 93.96(\mathrm{~s})$.

${ }^{19}$ F NMR (376 MHz, DMSO-d $) \delta-120.18(\mathrm{~d}, J=9.0 \mathrm{~Hz}, 2 \mathrm{~F})$.

ESI-HRMS: $m / z$ calcd. for $\left[\mathrm{C}_{9} \mathrm{H}_{6} \mathrm{BrF}_{2} \mathrm{~N}_{4}\right]^{+}: 286.9738$ found $286.9747\left([\mathrm{M}+\mathrm{H}]^{+}\right), m / z$ calcd. for $\left[\mathrm{C}_{9} \mathrm{H}_{6} \mathrm{BrF}_{2} \mathrm{~N}_{4}\right]^{+}: 288.9718$ found $288.9726\left([\mathrm{M}+2+\mathrm{H}]^{+}\right)$,

(E)-3-((4-bromo-2,6-difluorophenyl)diazenyl)-1-(2-fluoro-6-(trifluoromethyl)benzyl)-1H-pyrazole (piCRAC-10)<smiles>Fc1cccc(C(F)(F)F)c1Cn1ccc(N=Nc2c(F)cc(Br)cc2F)n1</smiles>

A yellow solid was obtained (12 mg, 14.89\%).

m.p: $92.8-94.5^{\circ} \mathrm{C}$.

TLC (PE:EA=10:1): $\mathbf{R}_{\mathbf{f}}=0.5$

${ }^{1}$ H NMR (400 MHz, MeOD-d $\left.d_{4}\right) 7.69(\mathrm{~d}, J=5.9 \mathrm{~Hz}, 2 \mathrm{H}), 7.56(\mathrm{dd}, J=11.6,6.1 \mathrm{~Hz}, 1 \mathrm{H}), 7.45(\mathrm{~d}, J=9.6$ $\mathrm{Hz}, 2 \mathrm{H}), 7.01(\mathrm{~d}, J=7.0 \mathrm{~Hz}, 1 \mathrm{H}), 6.62(\mathrm{~s}, 1 \mathrm{H}), 5.69(\mathrm{~s}, 2 \mathrm{H})$.

${ }^{13}$ C NMR (101 MHz, MeOD-d 4$) \delta 164.35(\mathrm{~s}), 162.55(\mathrm{~d}, J=249.9 \mathrm{~Hz}), 155.48(\mathrm{dd}, J=262.8,5.1 \mathrm{~Hz}$, 2C), $132.13(\mathrm{~s}), 131.41$ (d, $J=9.5 \mathrm{~Hz}), 131.18$ (d, $J=9.5 \mathrm{~Hz}), 126.00(\mathrm{dd}, J=526.4,367.3 \mathrm{~Hz}), 122.92$ $122.55(\mathrm{~m}), 122.37-121.92(\mathrm{~m}), 120.30(\mathrm{~d}, J=16.7 \mathrm{~Hz}), 119.91$ (d, $J=23.2 \mathrm{~Hz}), 116.35(\mathrm{dd}, J=24.6,3.2$ $\mathrm{Hz}, 2 \mathrm{C}), 115.31$ (d, $J=27.4 \mathrm{~Hz}), 94.37$ (s), 46.15 (s).

${ }^{19}$ F NMR (376 MHz, MeOD-d $)_{4} \delta-59.91$ (s, 3F), -114.12 (d, $\left.J=8.9 \mathrm{~Hz}, 1 \mathrm{~F}\right),-120.91$ (d, $\left.J=9.1 \mathrm{~Hz}, 2 \mathrm{~F}\right)$. 
ESI-HRMS: $m / z$ calcd. for $\left[\mathrm{C}_{17} \mathrm{H}_{10} \mathrm{BrF}_{6} \mathrm{~N}_{4}\right]^{+}: 462.9988$ found $462.9981\left([\mathrm{M}+\mathrm{H}]^{+}\right), m / z$ calcd. for $\left[\mathrm{C}_{17} \mathrm{H}_{10} \mathrm{BrF}_{6} \mathrm{~N}_{4}\right]^{+}: 464.9967$ found $464.9962\left([\mathrm{M}+2+\mathrm{H}]^{+}\right)$,

HPLC: mobile phase acetonitrile: $\mathrm{H}_{2} \mathrm{O}(75: 25)$, flow velocity $(1.0 \mathrm{~mL} / \mathrm{min}) . \mathrm{Sig}=302 \mathrm{~nm}, 4 \mathrm{~nm}$, purity of trans $86.12 \%$. 
NMR, MS, HPLC Spectra of piCRACs.

(E)-3-((2,6-difluorophenyl)diazenyl)-1H-pyrazole (1-3)

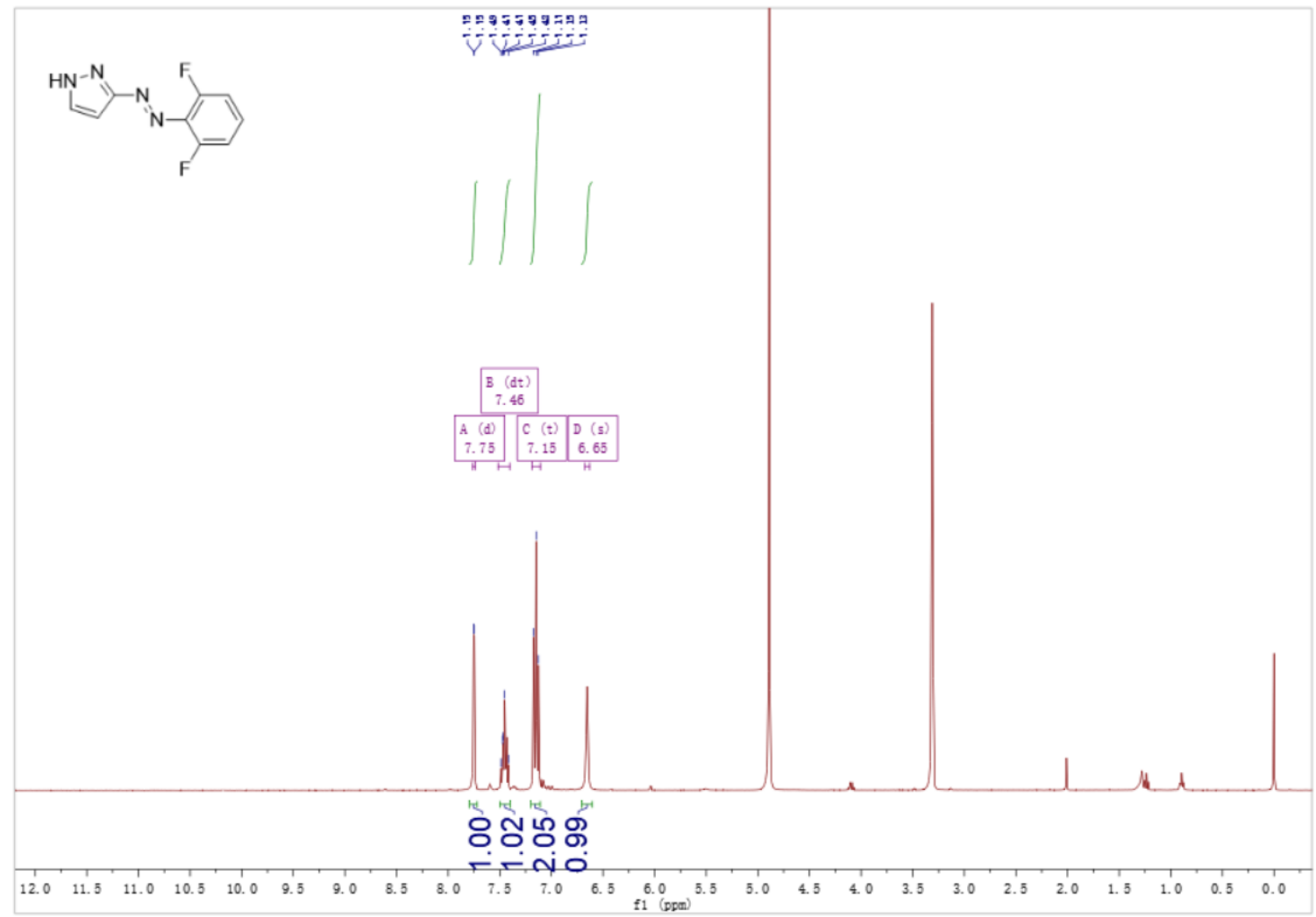



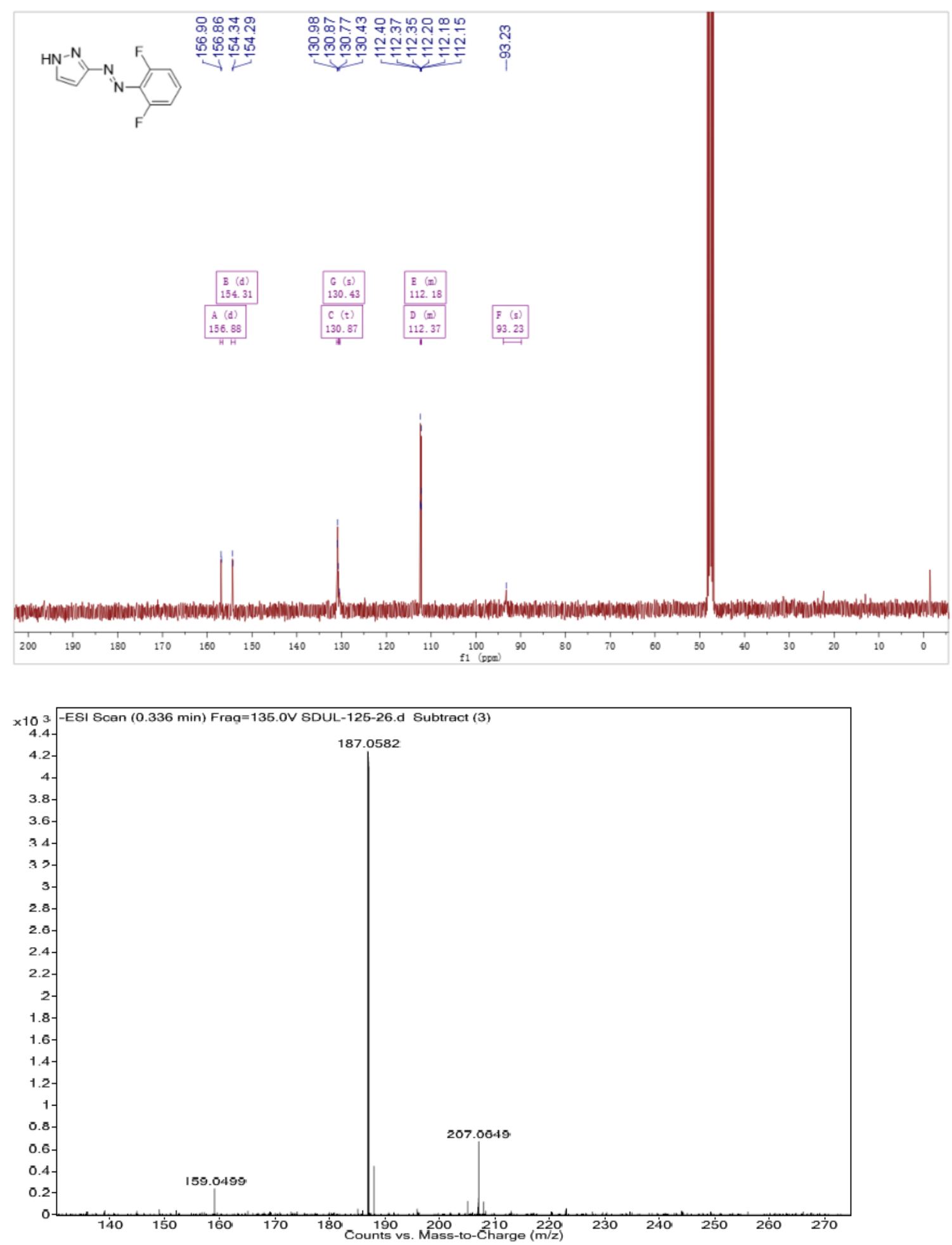

(E)-3-((2,6-difluorophenyl)diazenyl)-1-(2-fluoro-6-(trifluoromethyl)benzyl)-1H-pyrazole (piCRAC-1) 


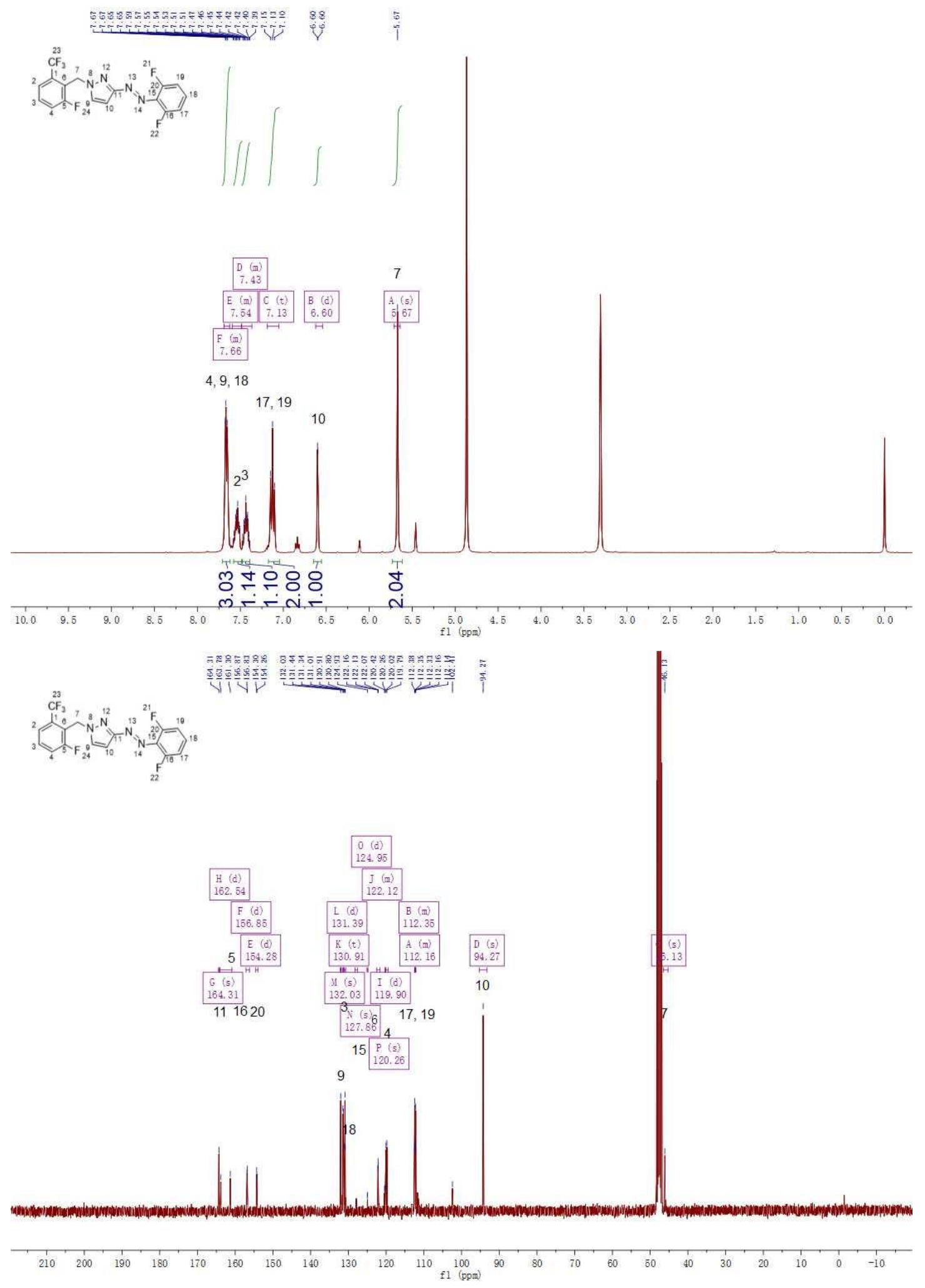



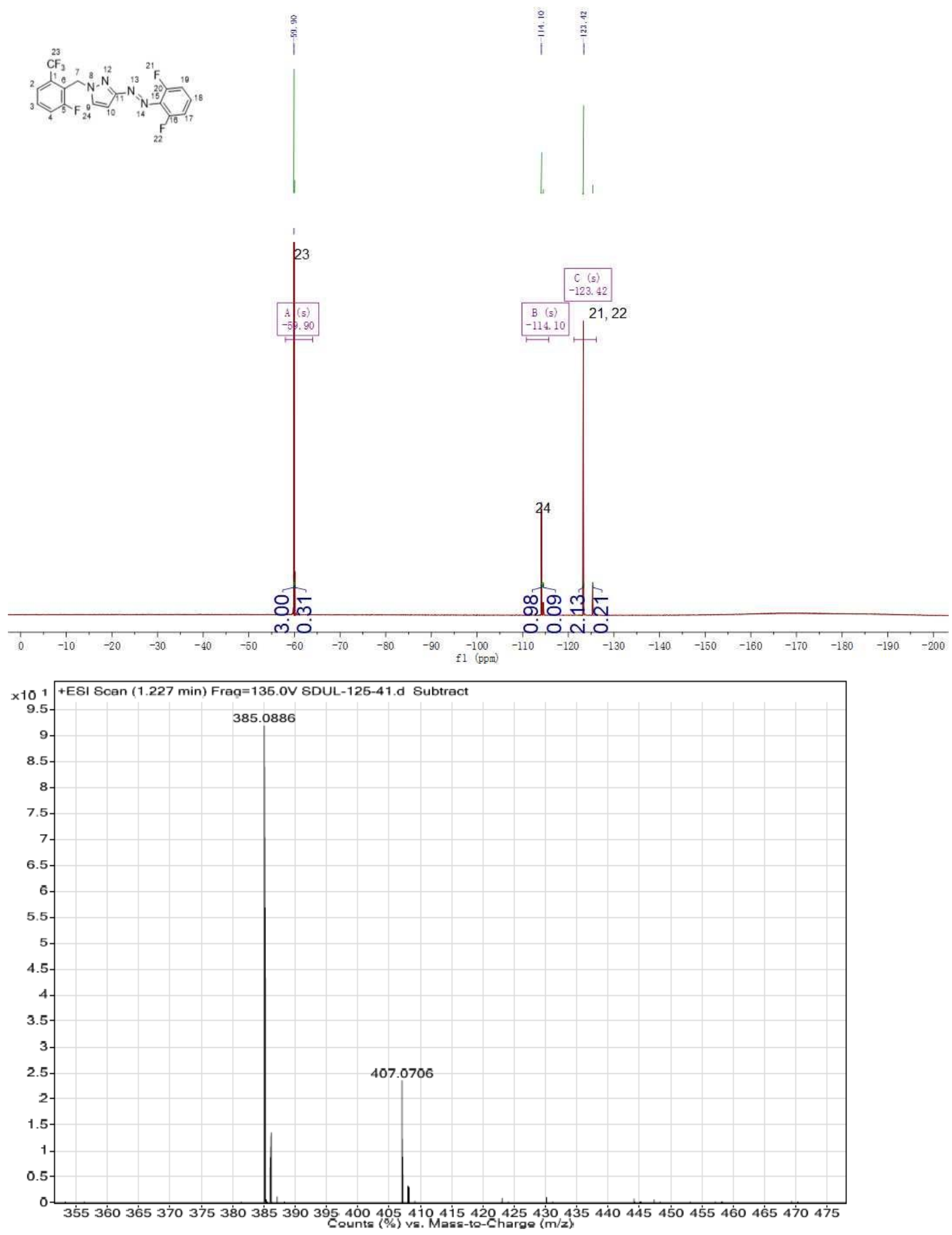


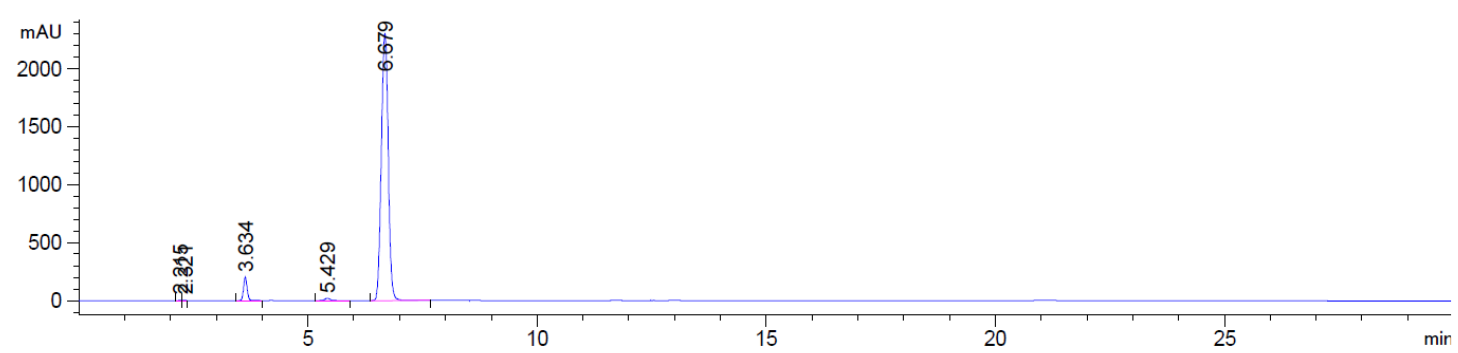

4-(hydroxymethyl)-3-(trifluoromethyl)phenol (2-2)

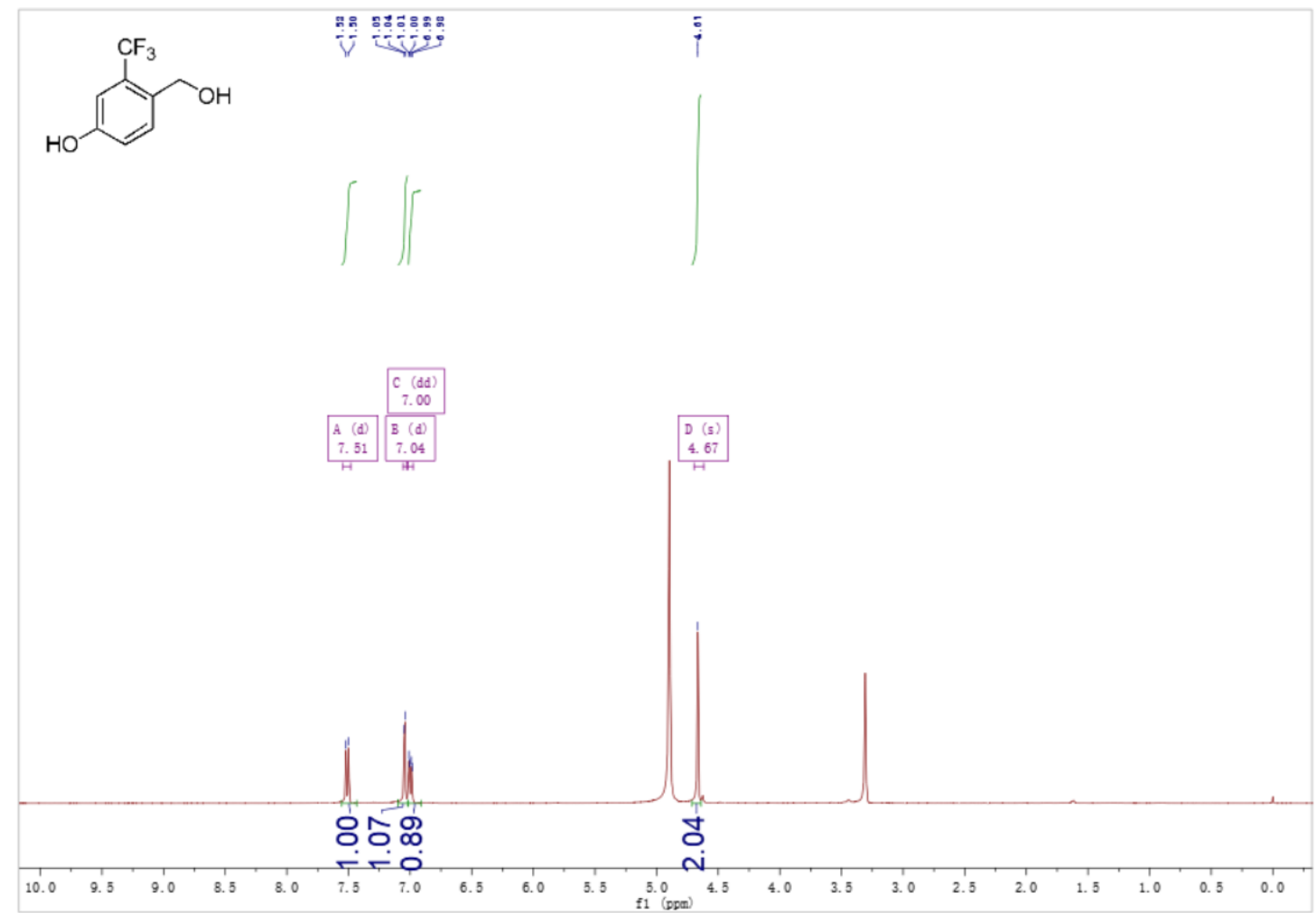



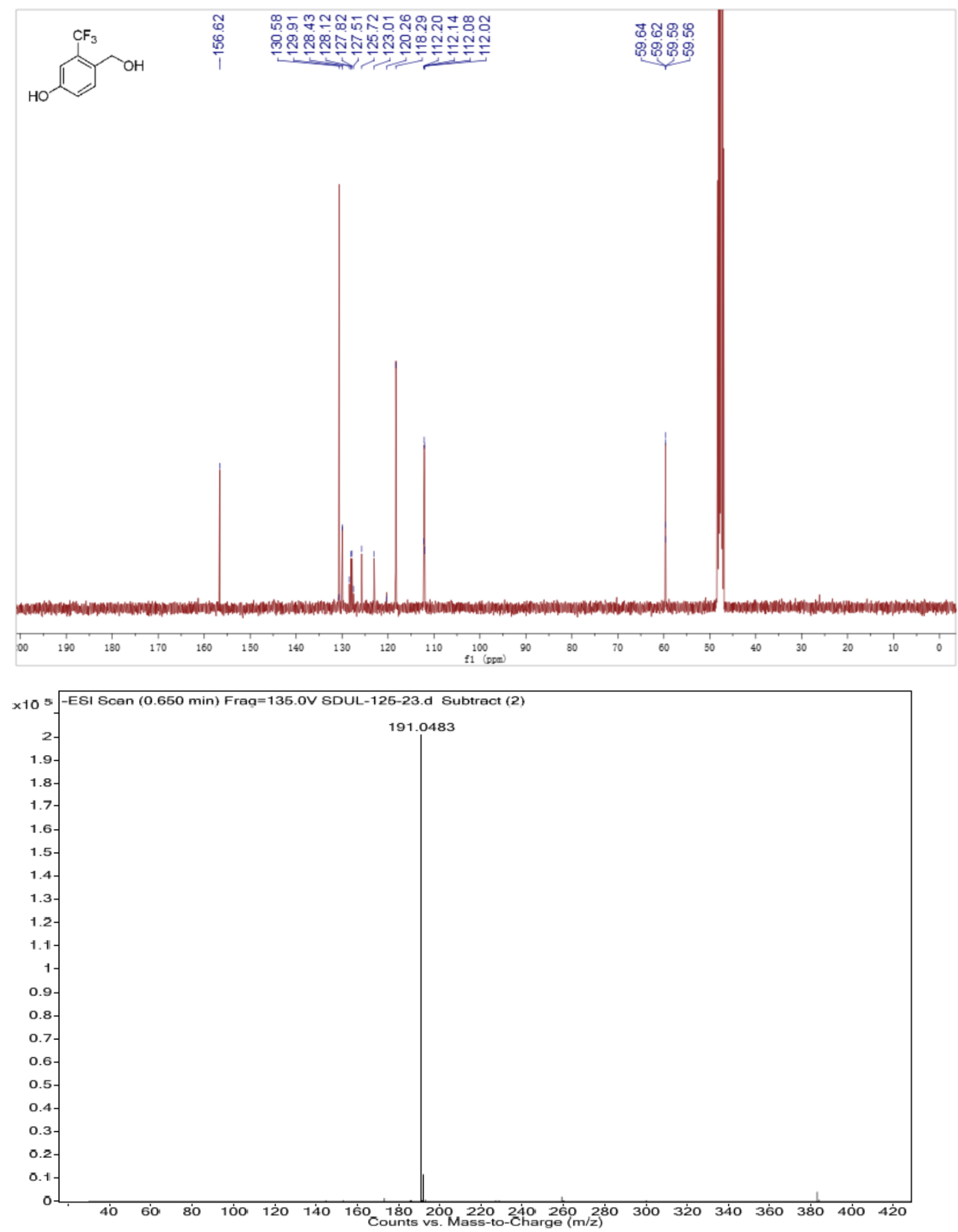

(4-(benzyloxy)-2-(trifluoromethyl)phenyl)methanol (2-3) 

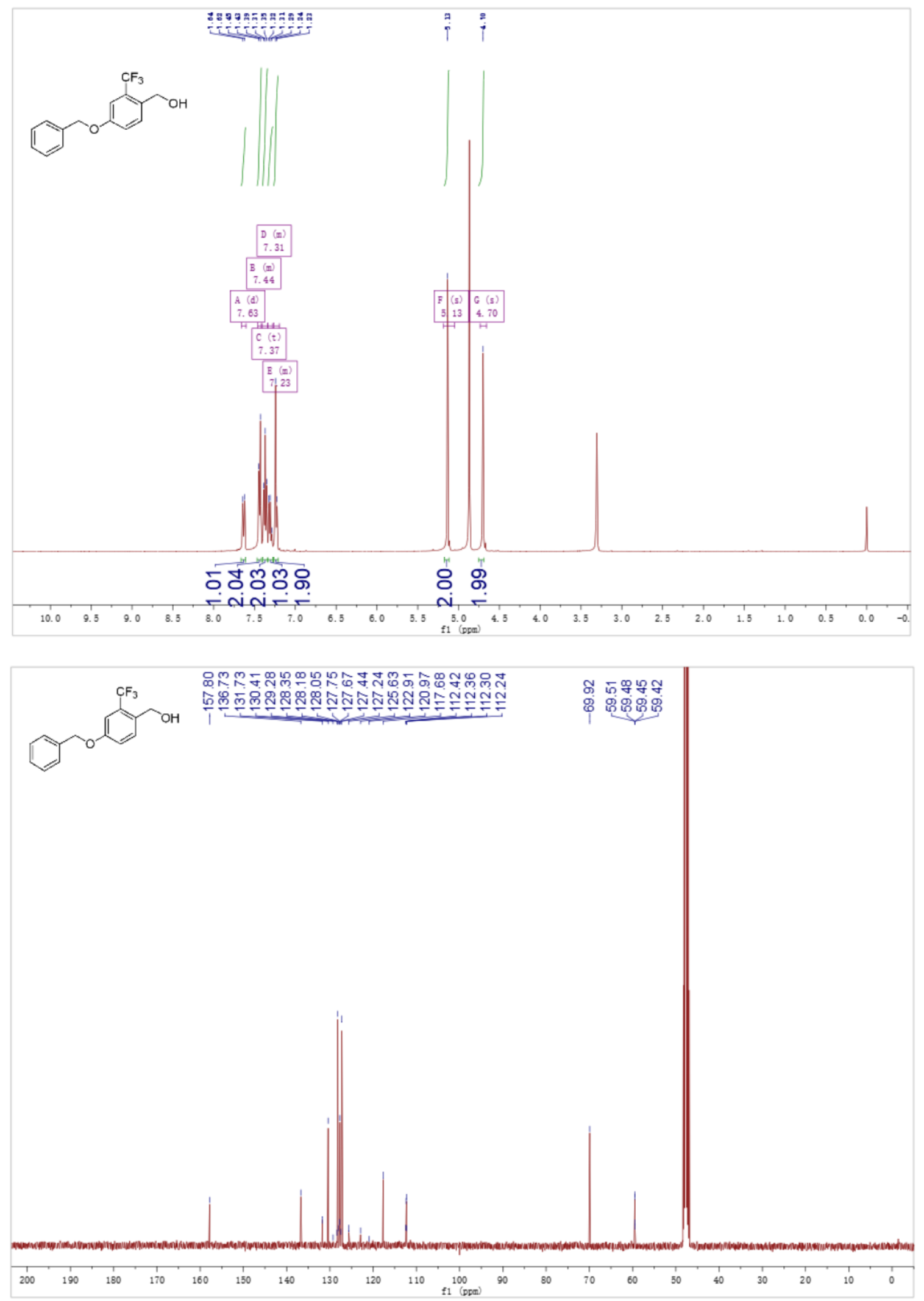

4-(benzyloxy)-1-(bromomethyl)-2-(trifluoromethyl)benzene (2-4) 


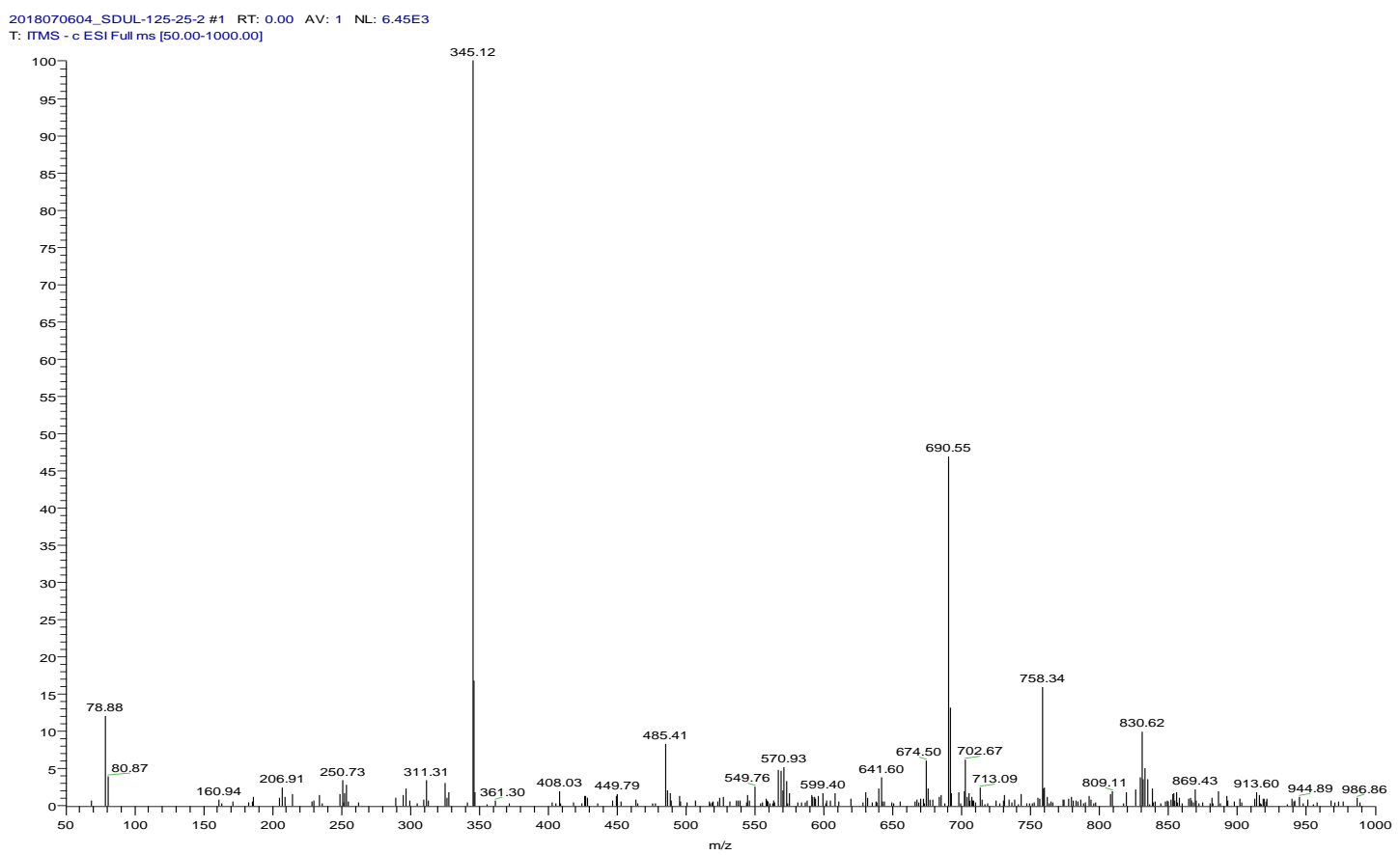

(E)-1-(4-(benzyloxy)-2-(trifluoromethyl)benzyl)-3-((2,6-difluorophenyl)diazenyl)-1H-pyrazole (2-5)

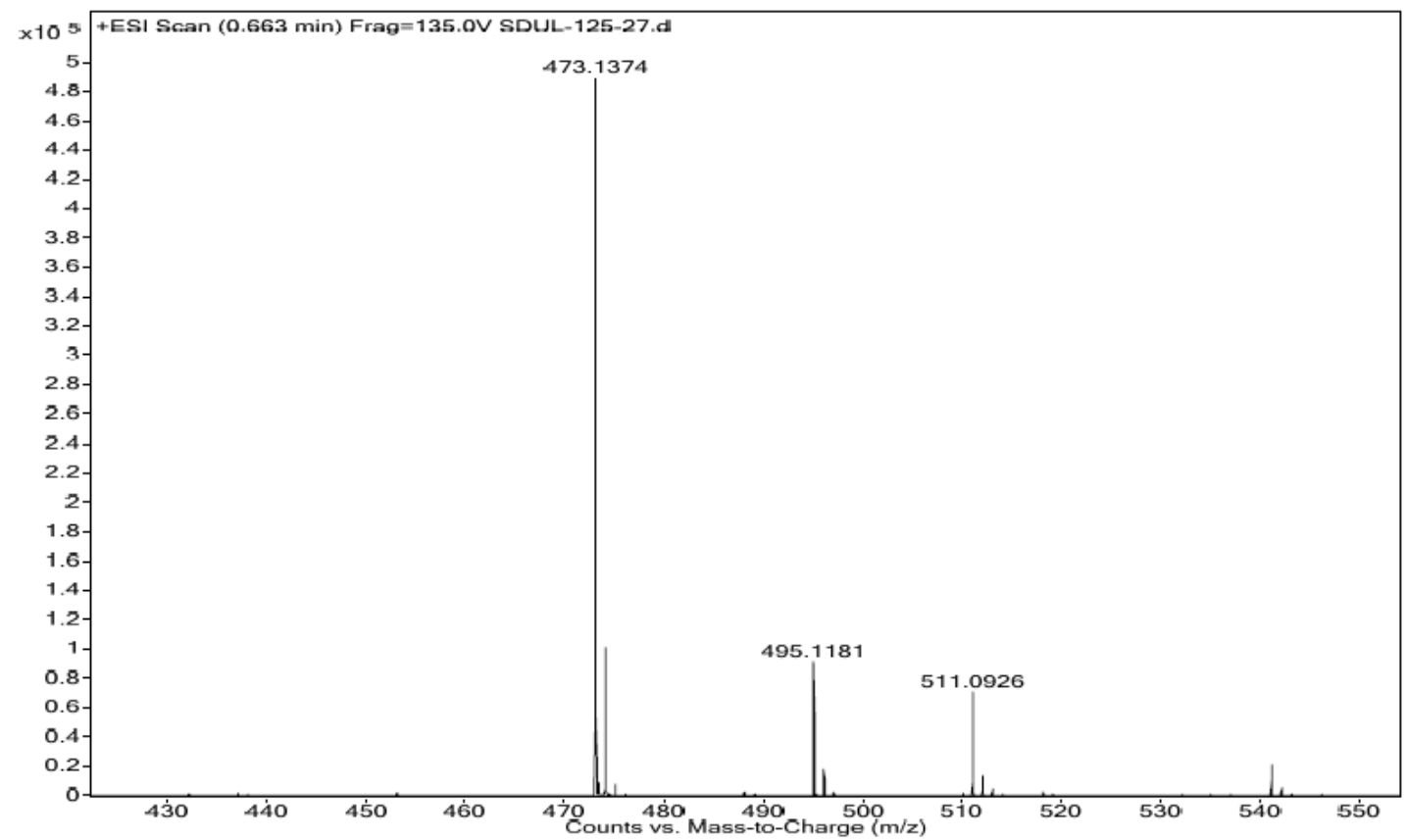

(E)-4-((3-((2,6-difluorophenyl)diazenyl)-1H-pyrazol-1-yl)methyl)-3-(trifluoromethyl)phenol (piCRAC-2) 

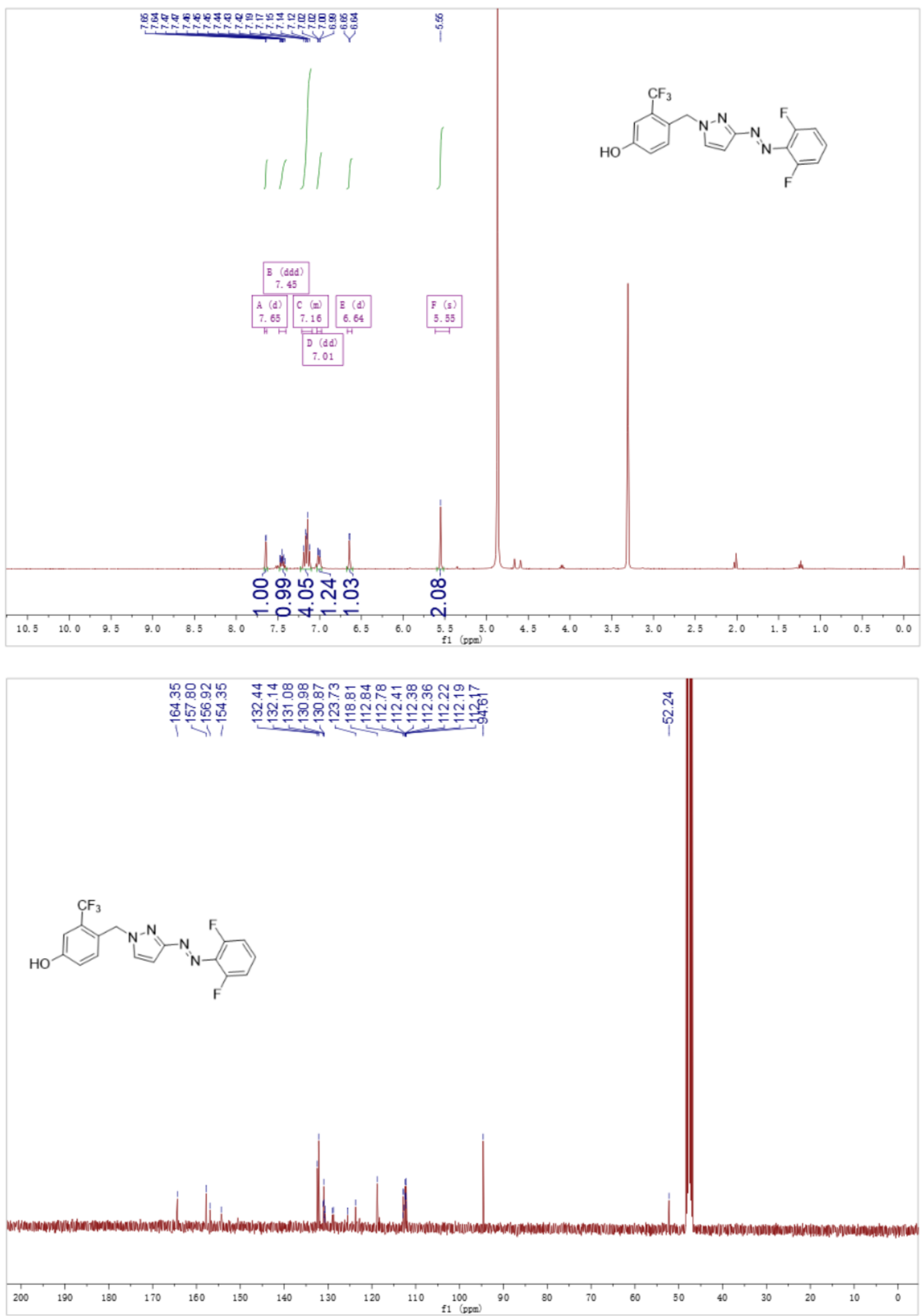

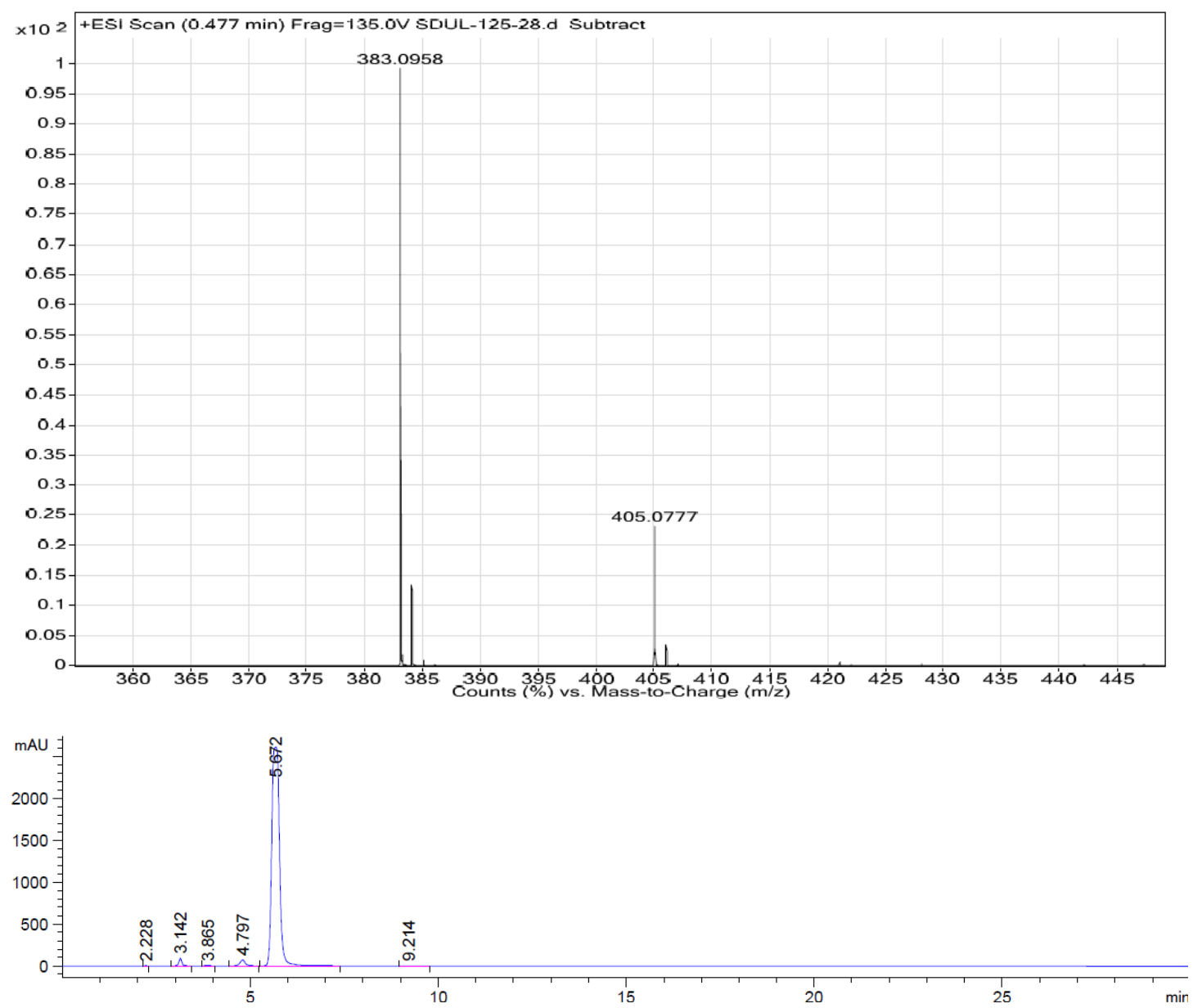

2-(hydroxymethyl)-5-(trifluoromethyl)phenol (3-2) 

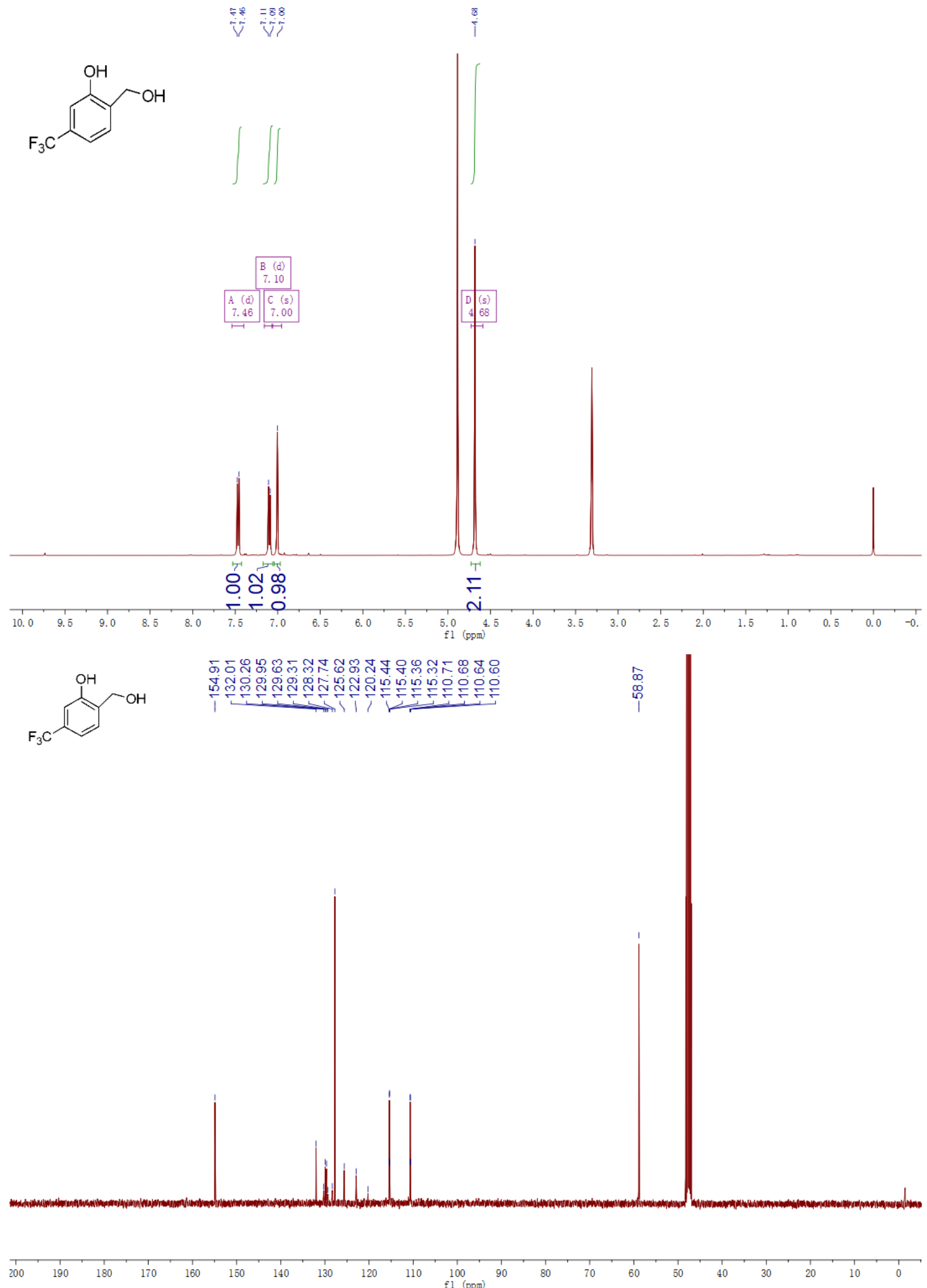


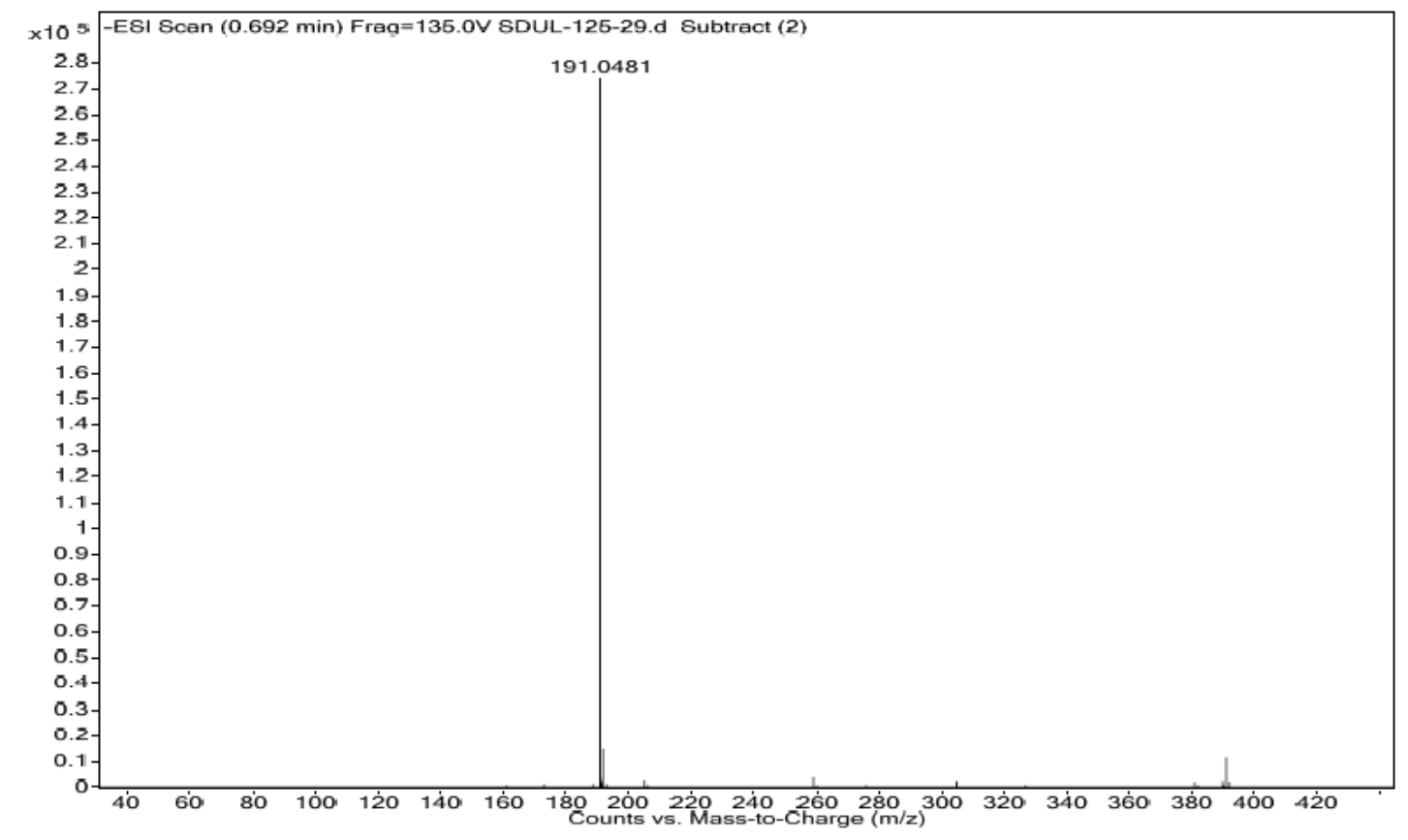

\section{(2-(benzyloxy)-4-(trifluoromethyl)phenyl)methanol (3-3)}
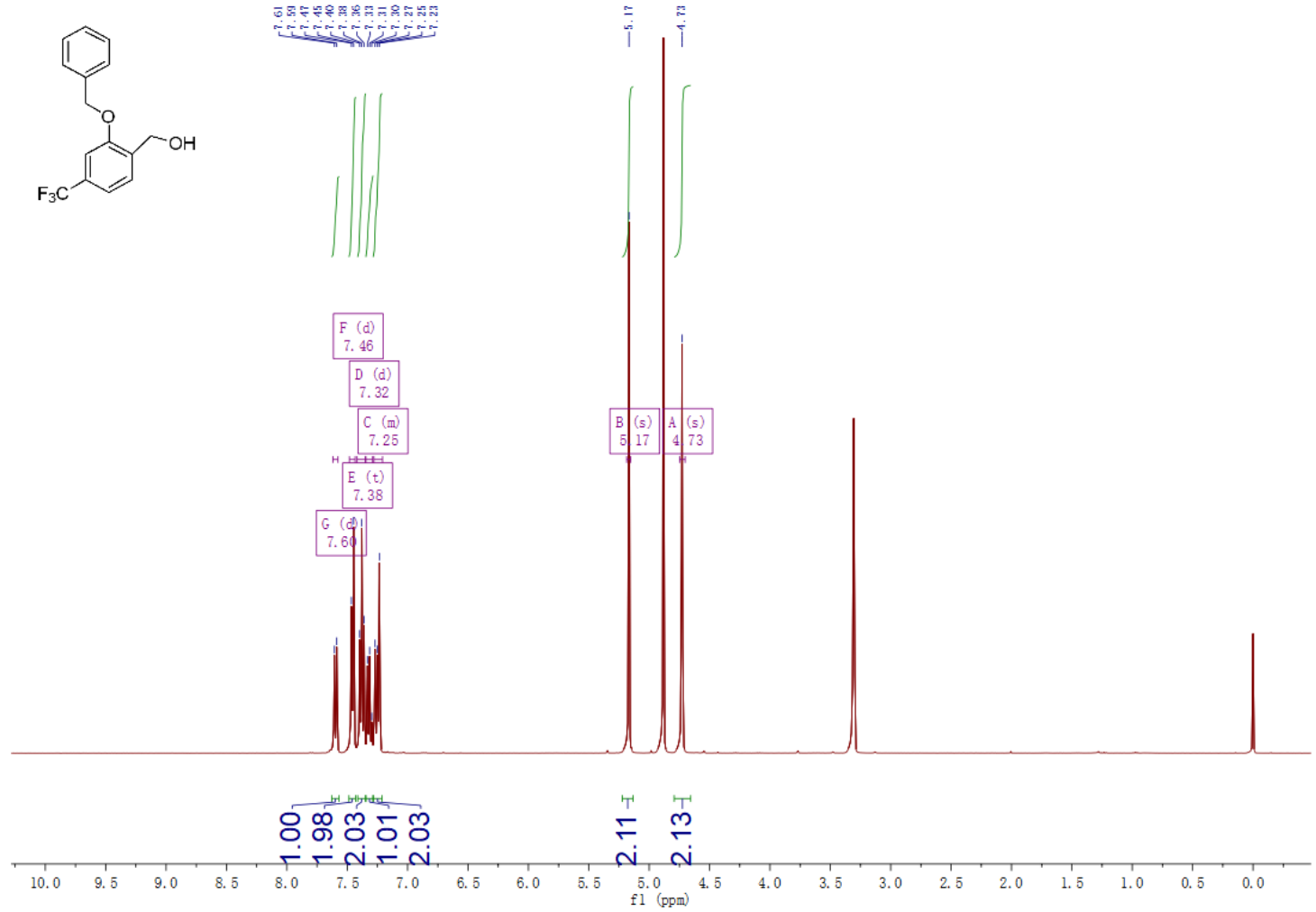

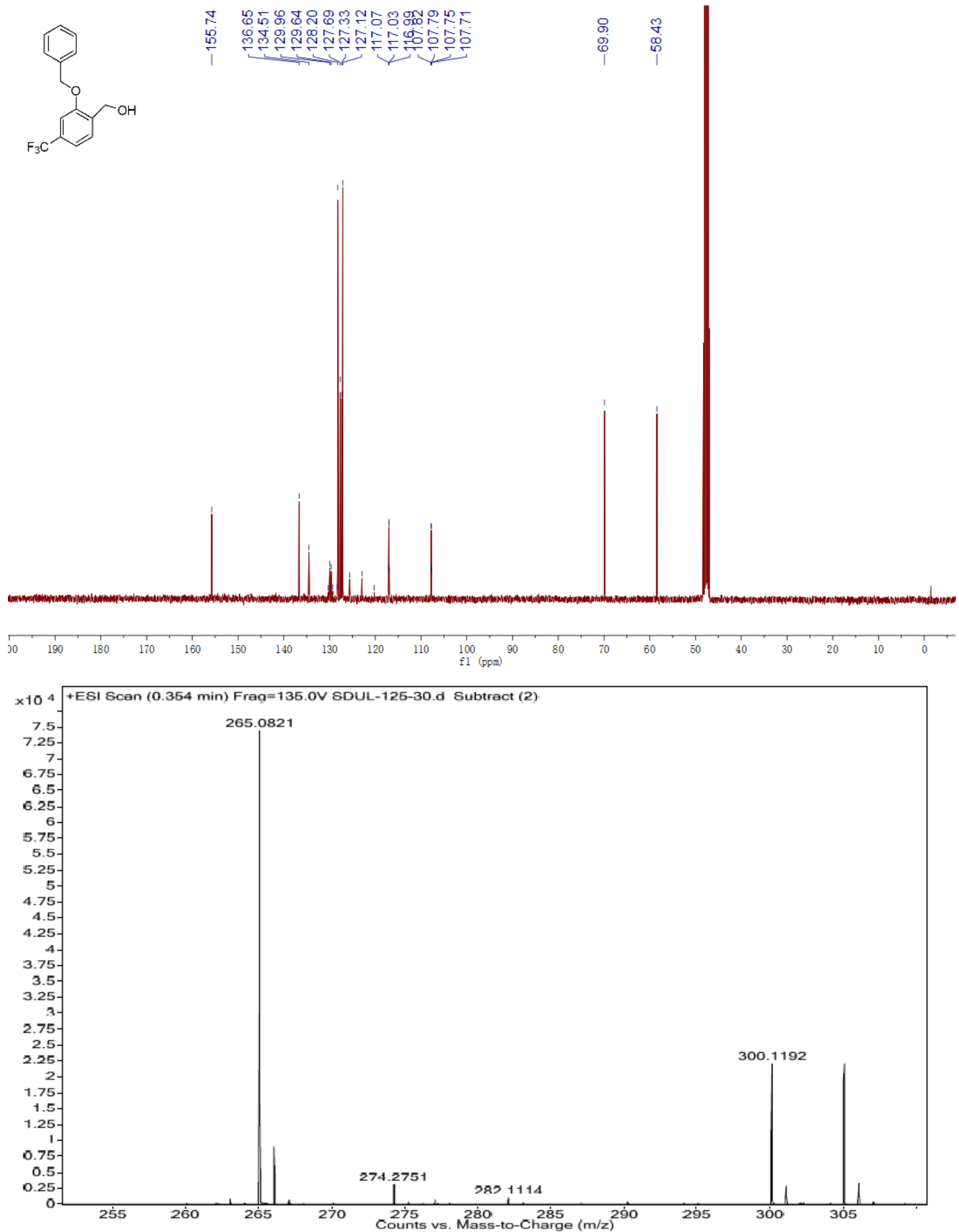

2-(benzyloxy)-1-(bromomethyl)-4-(trifluoromethyl)benzene (3-4) 

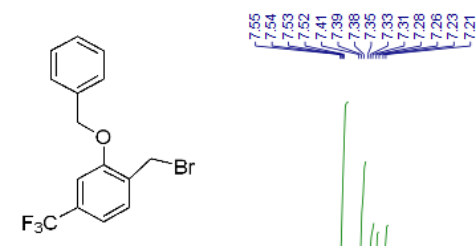

苟
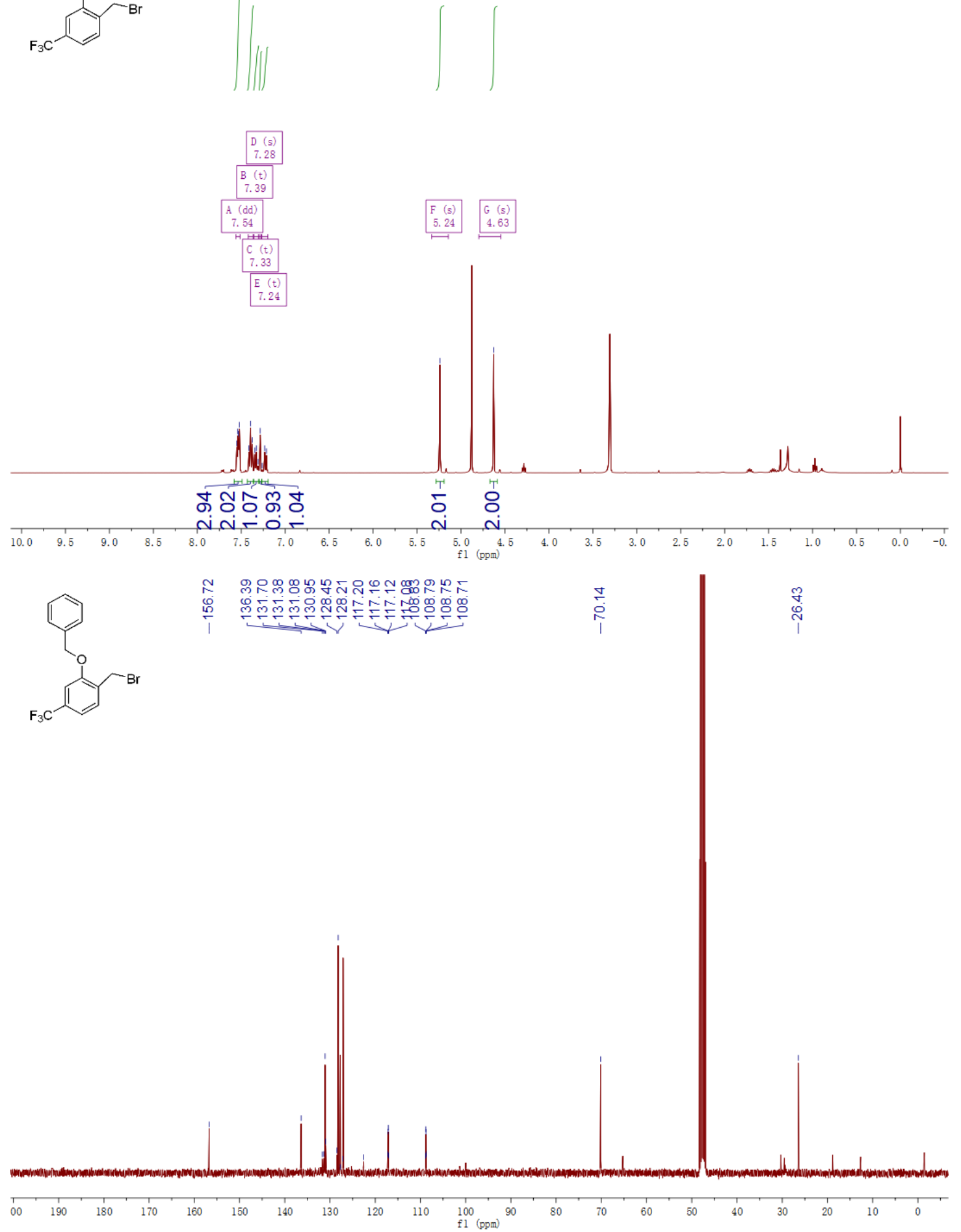

(E)-1-(2-(benzyloxy)-4-(trifluoromethyl)benzyl)-3-((2,6-difluorophenyl)diazenyl)-1H-pyrazole (3-5) 


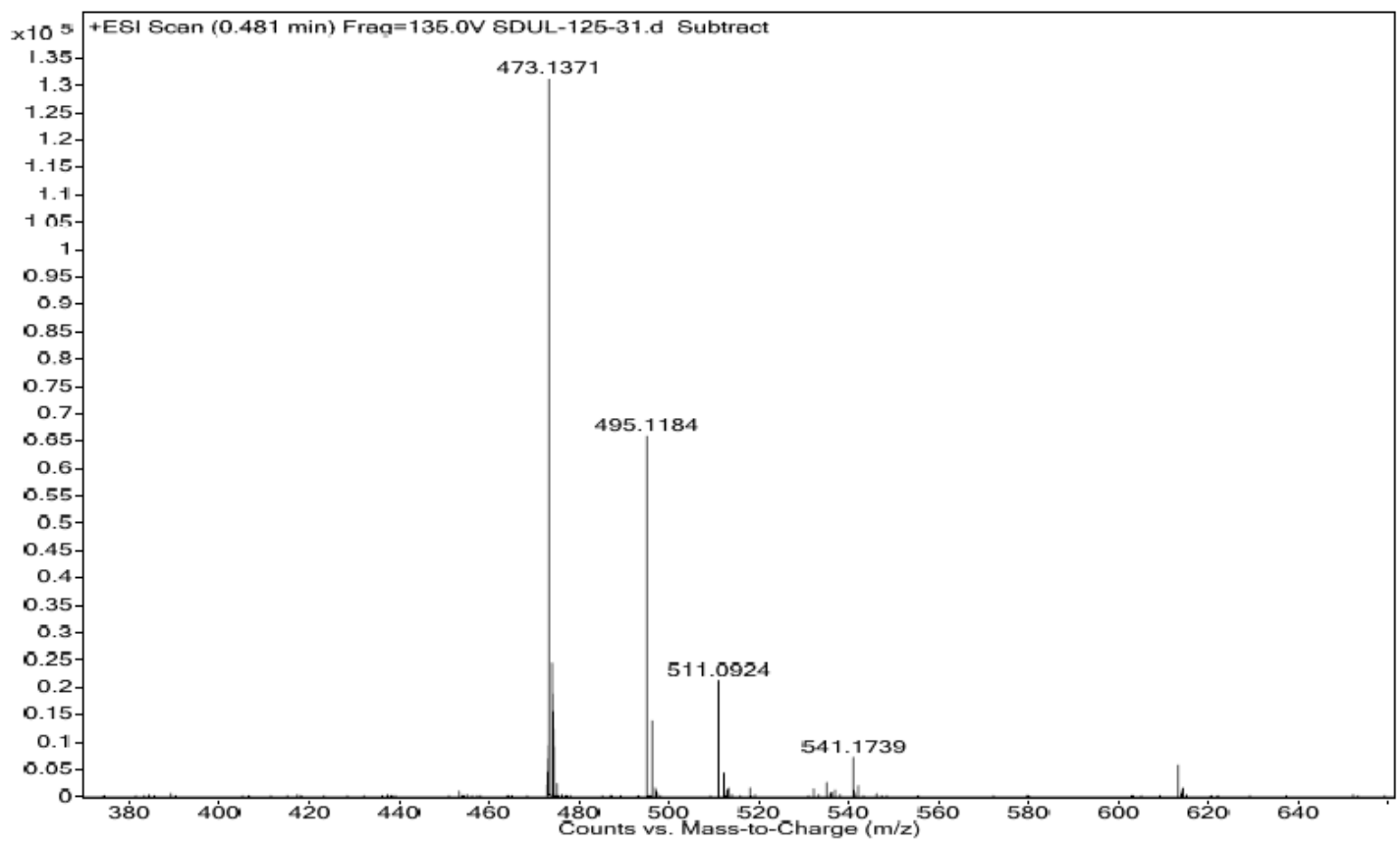

(E)-2-((3-((2,6-difluorophenyl)diazenyl)-1H-pyrazol-1-yl)methyl)-5-(trifluoromethyl)phenol (piCRAC-3)

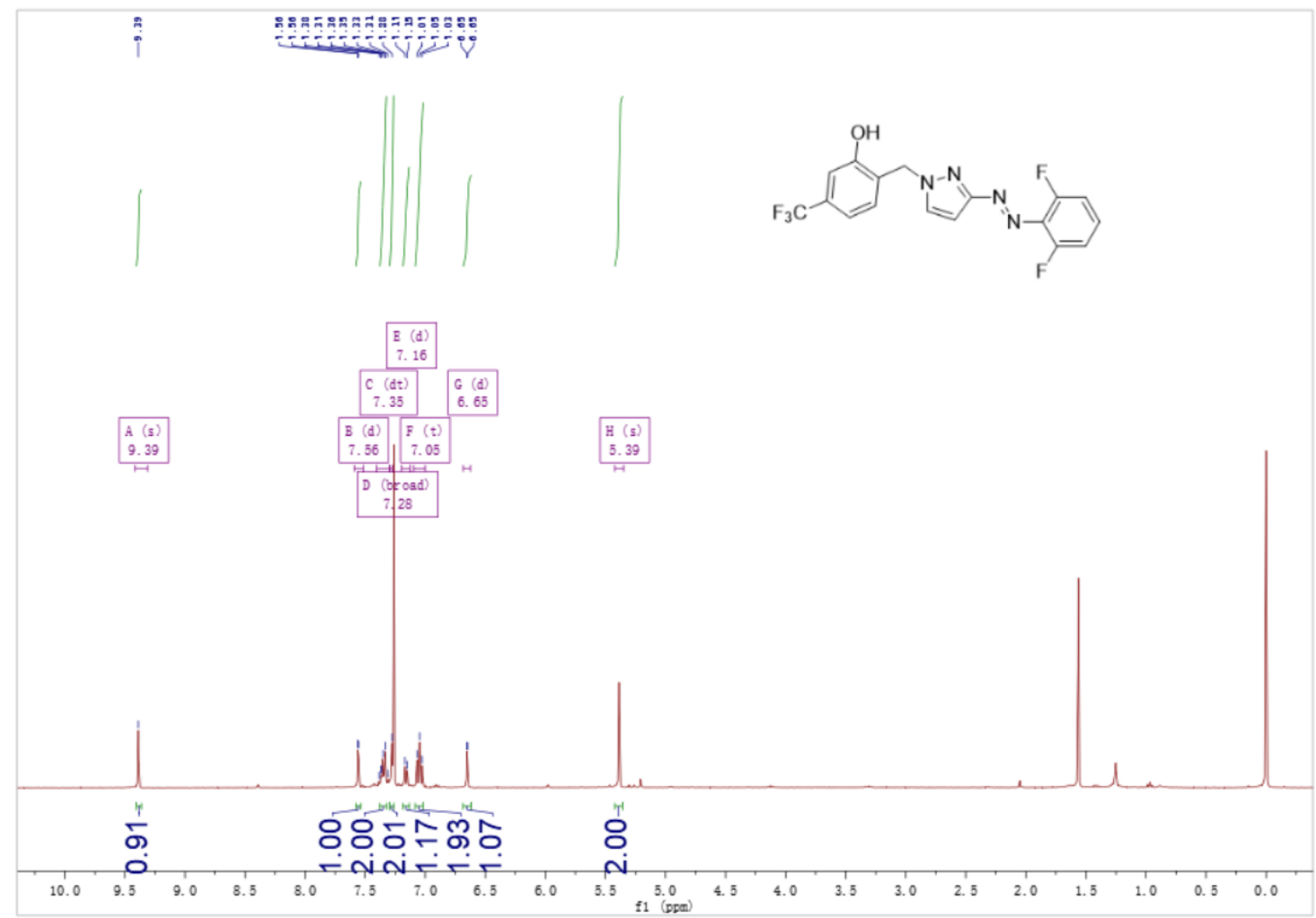



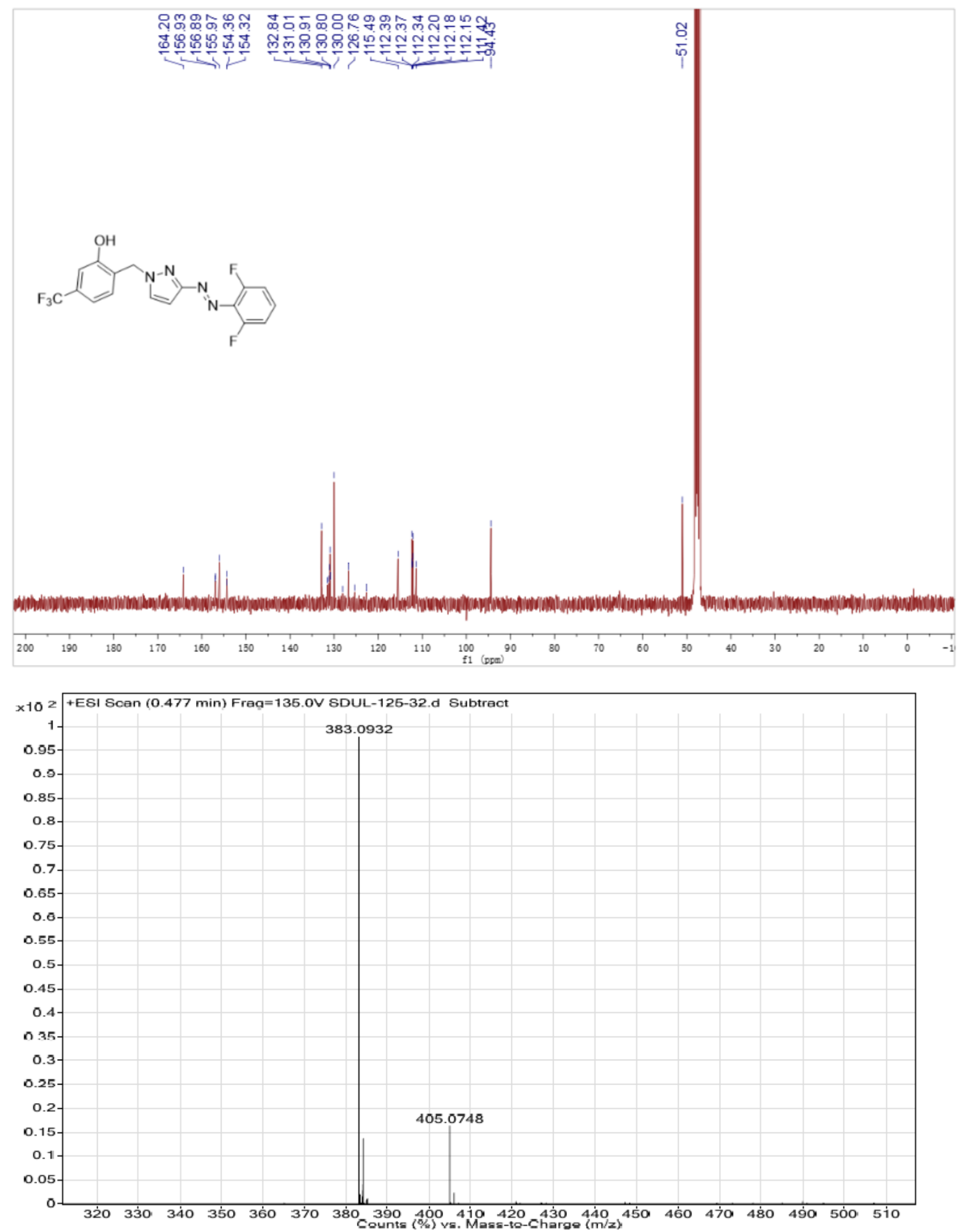


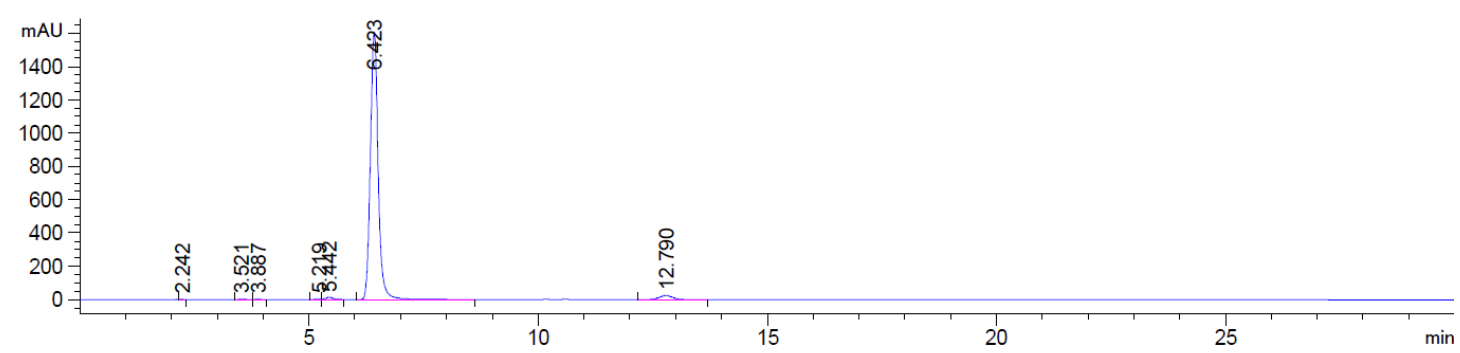

\section{2-phenoxybenzaldehyde (4-3)}

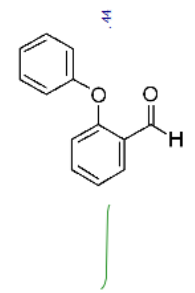

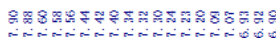

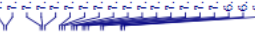

A (s)

F (m)
7.22

\begin{tabular}{|c||c|}
\hline C $(\mathrm{t})$ & G (d) \\
7. 58 & 7.08 \\
\hline
\end{tabular}

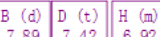

\begin{tabular}{llll}
7.89 & 7.42 & 6.92 \\
\hline
\end{tabular}

E (m)
7.32

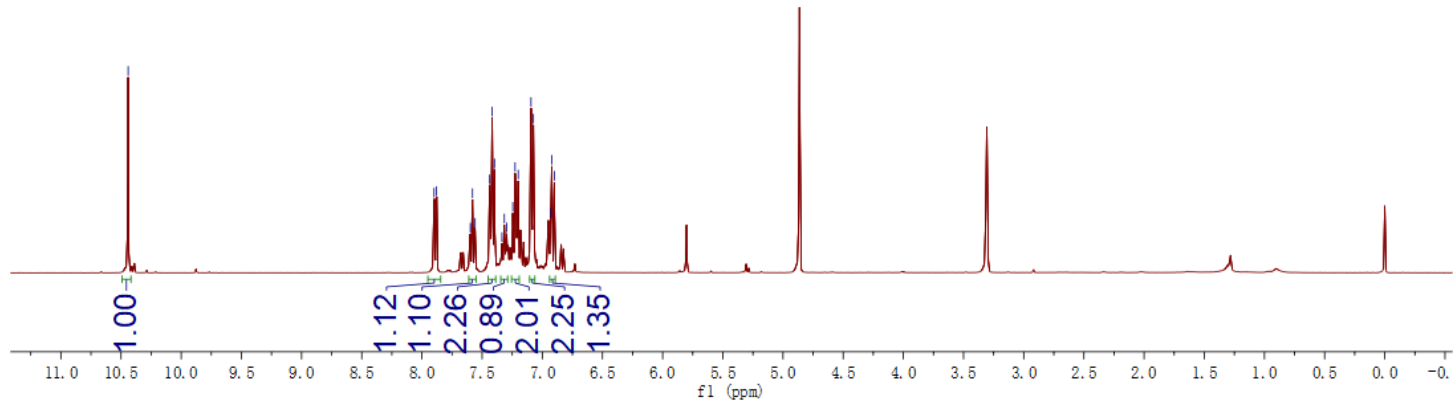



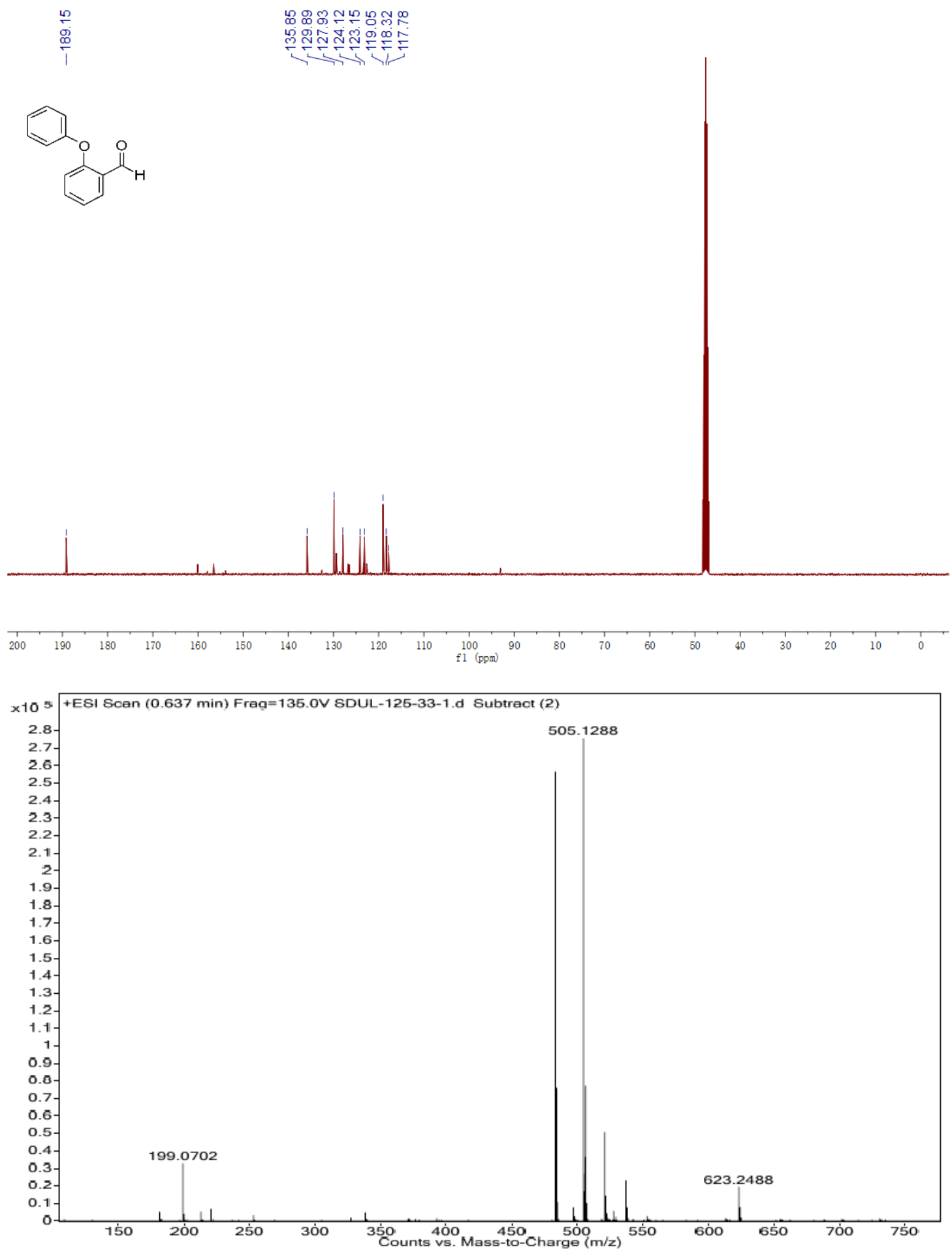

(2-phenoxyphenyl)methanol (4-4) 

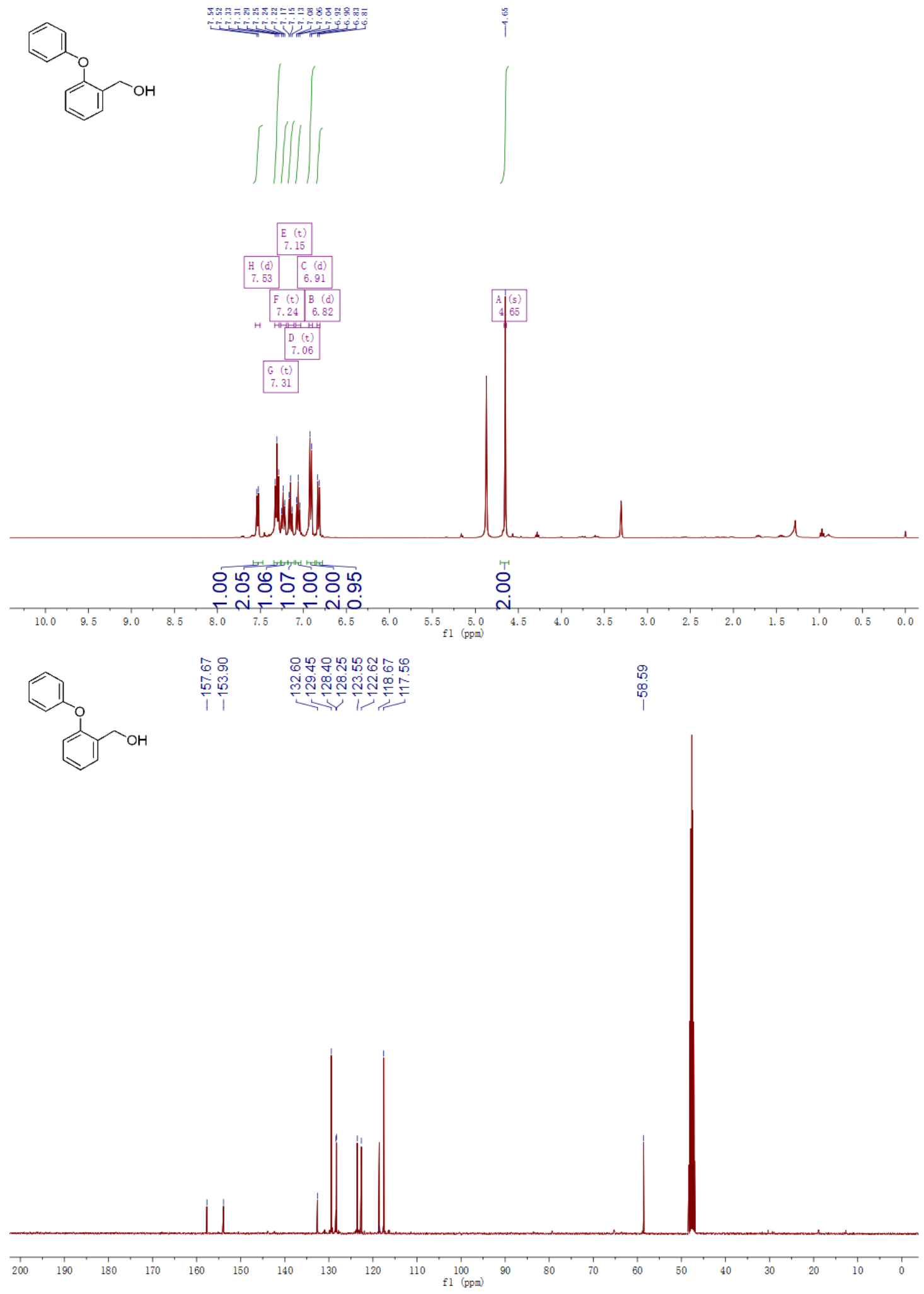


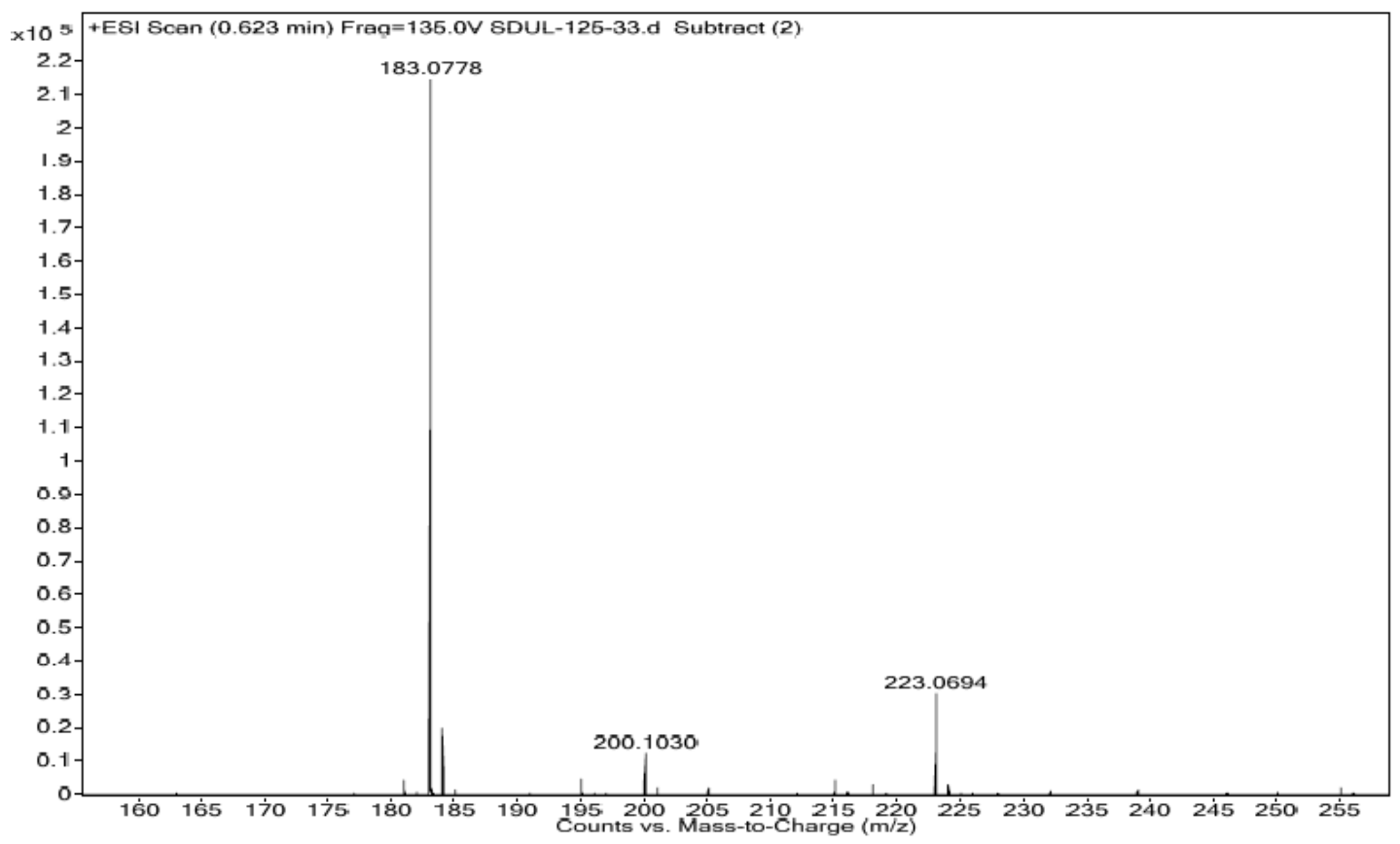

\section{1-(bromomethyl)-2-phenoxybenzene (4-5)}

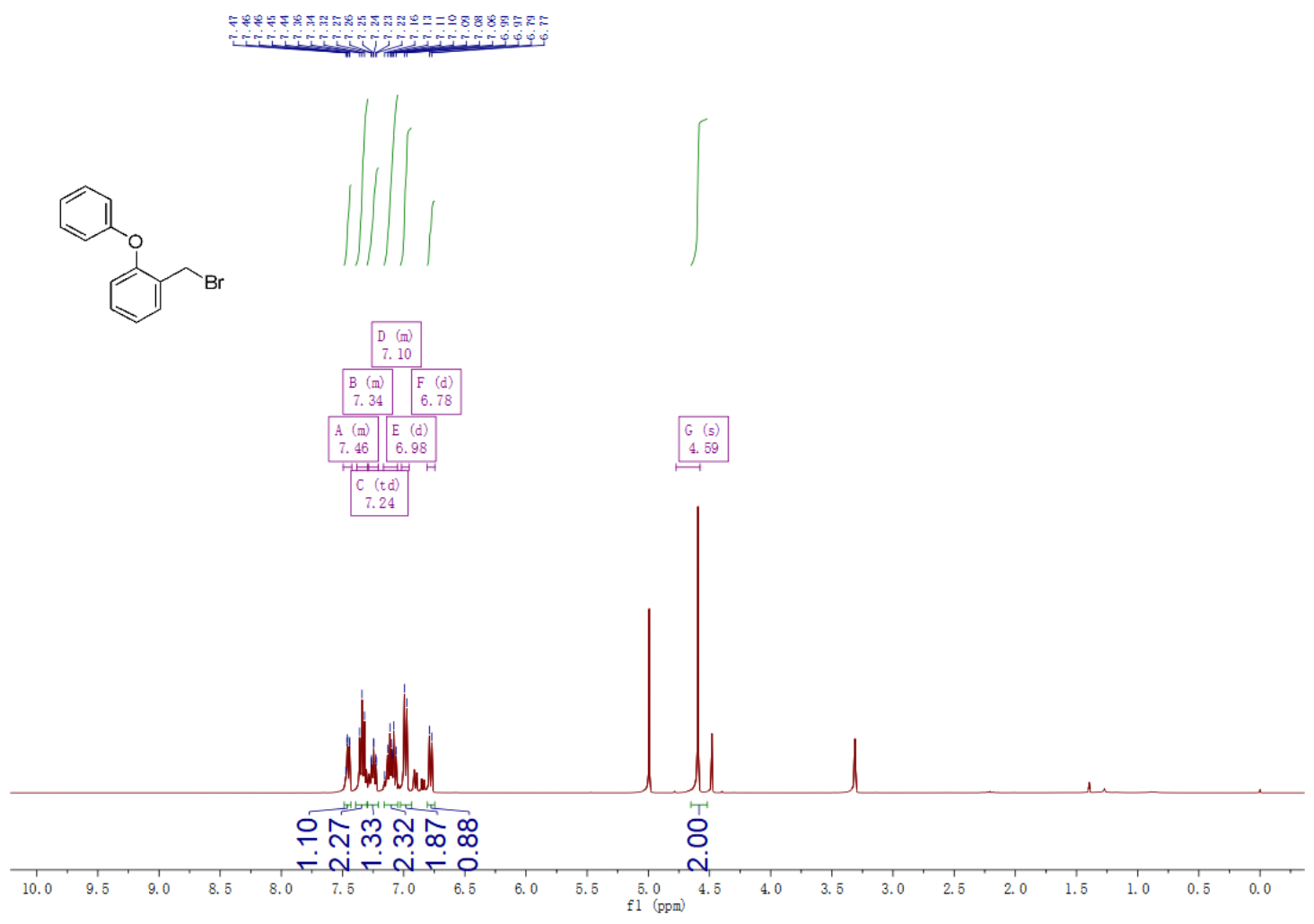


至

$\underset{i}{\stackrel{\infty}{i}}$
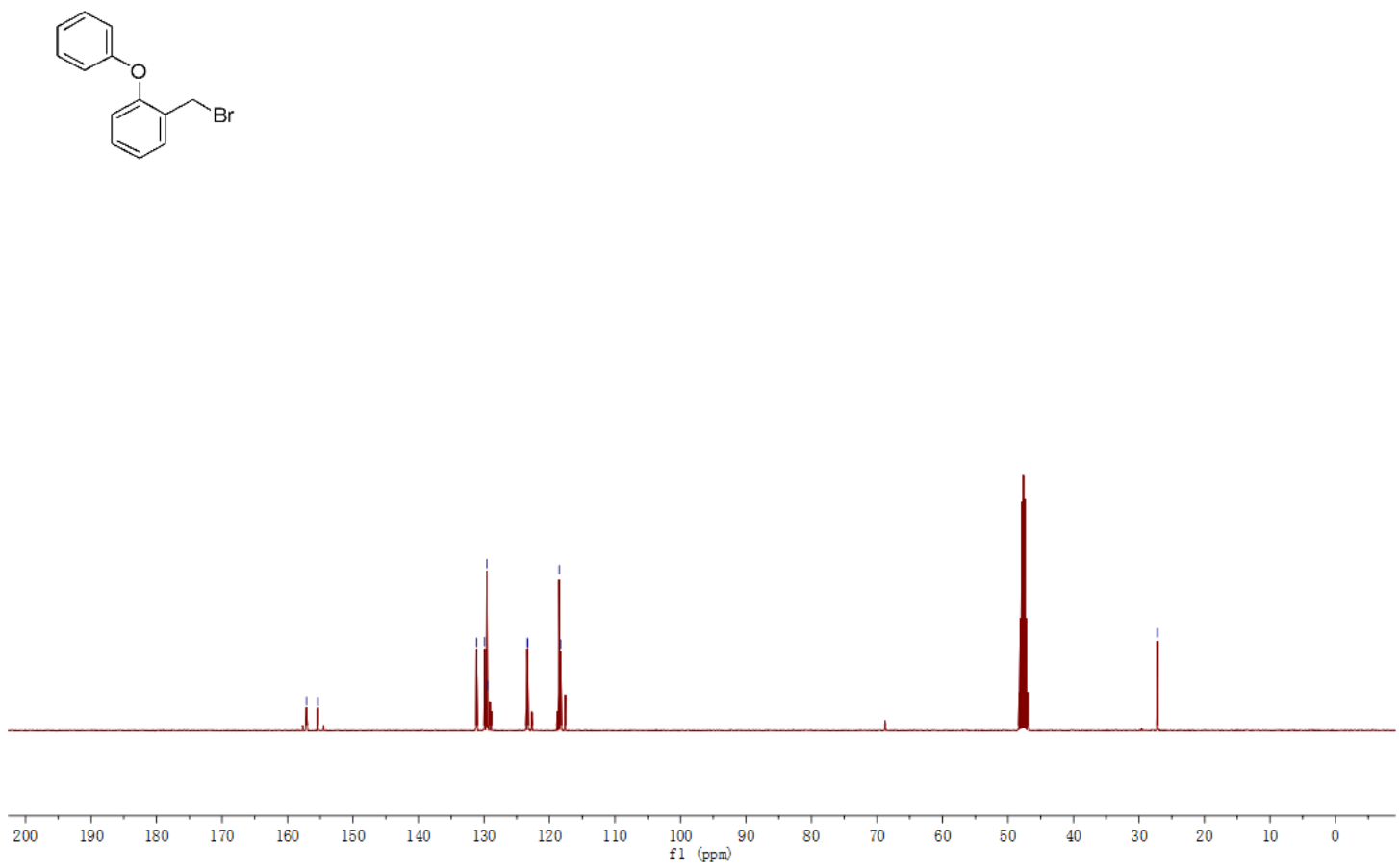

(E)-3-((2,6-difluorophenyl)diazenyl)-1-(2-phenoxybenzyl)-1H-pyrazole (piCRAC-4)

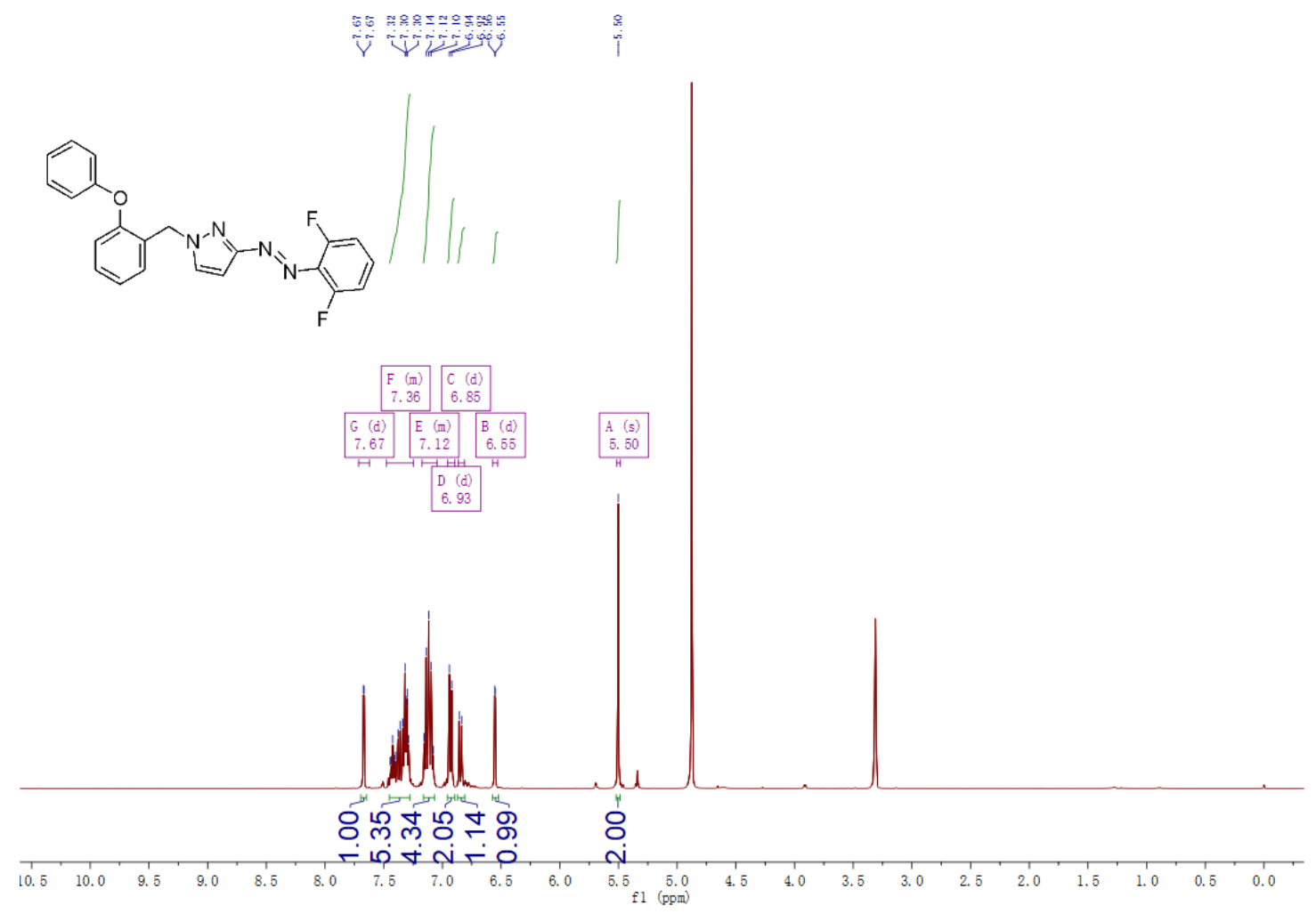

S54 

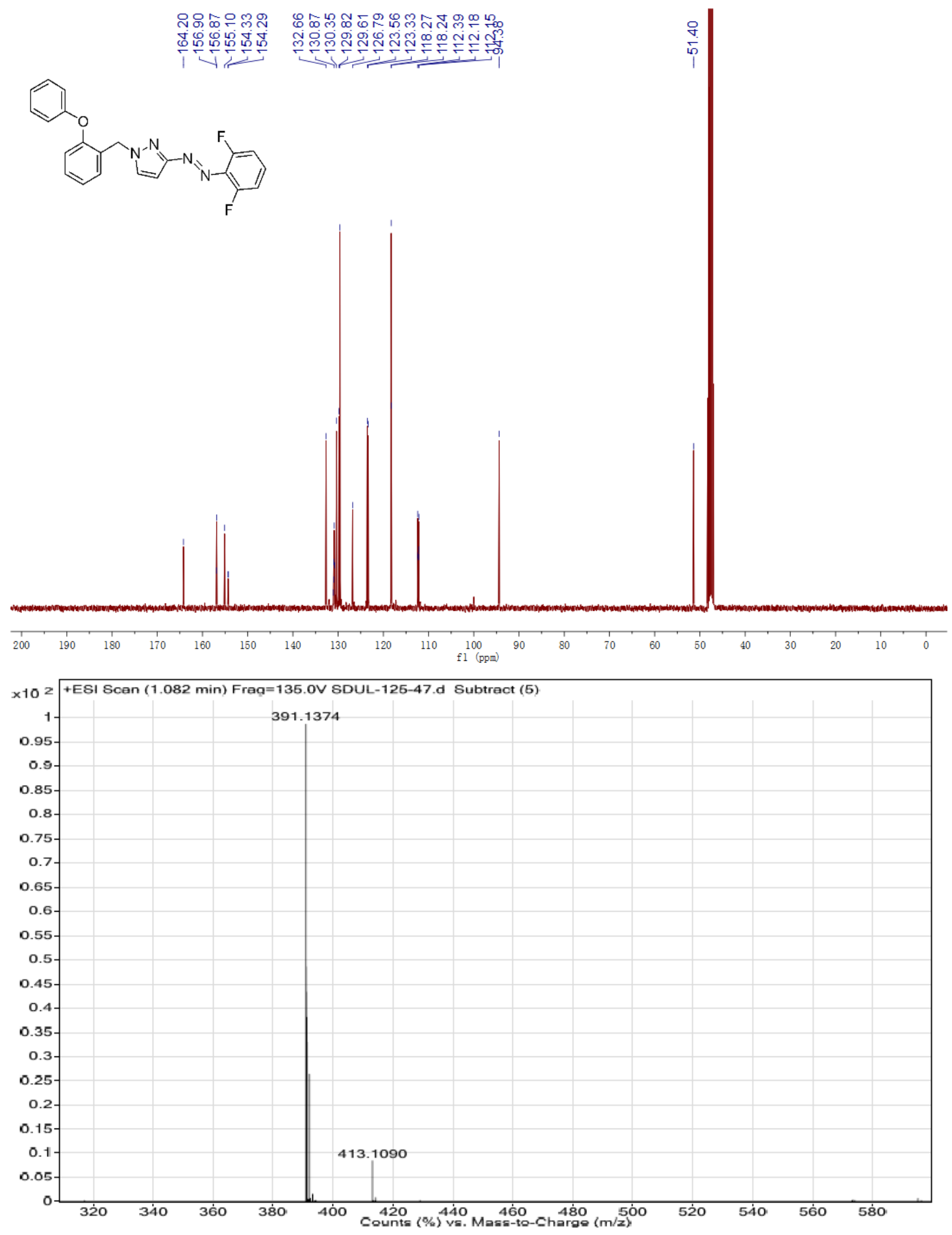


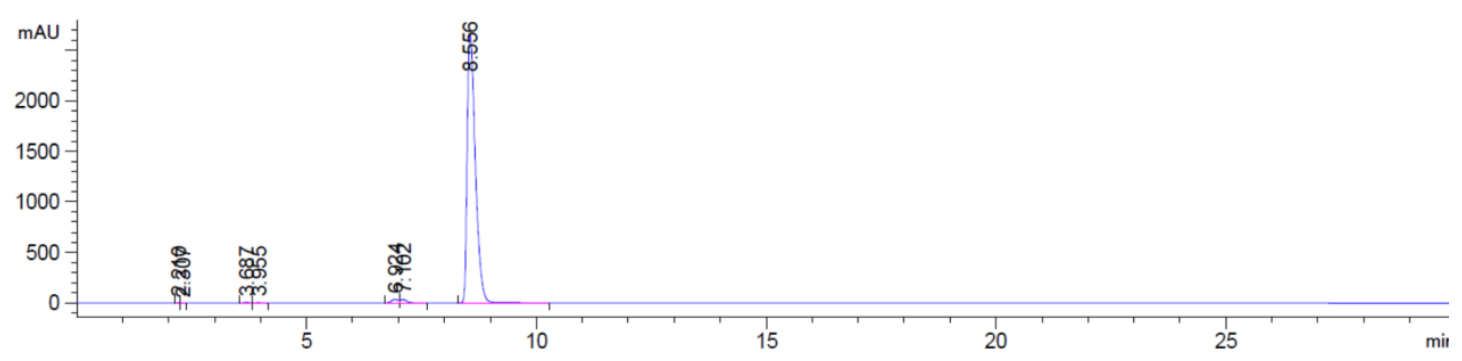

\section{2,5-dimethoxy-4'-nitro-1,1'-biphenyl (5-3)}
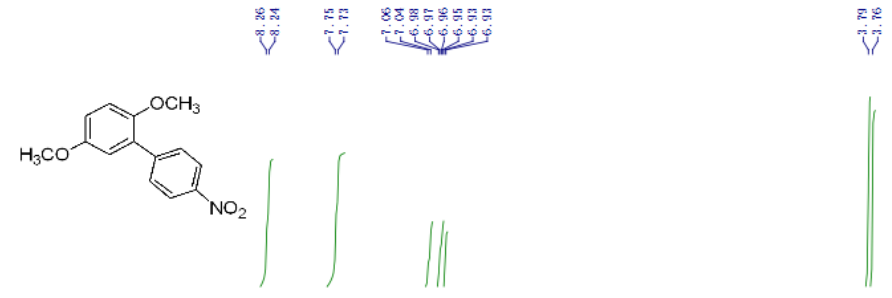

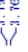

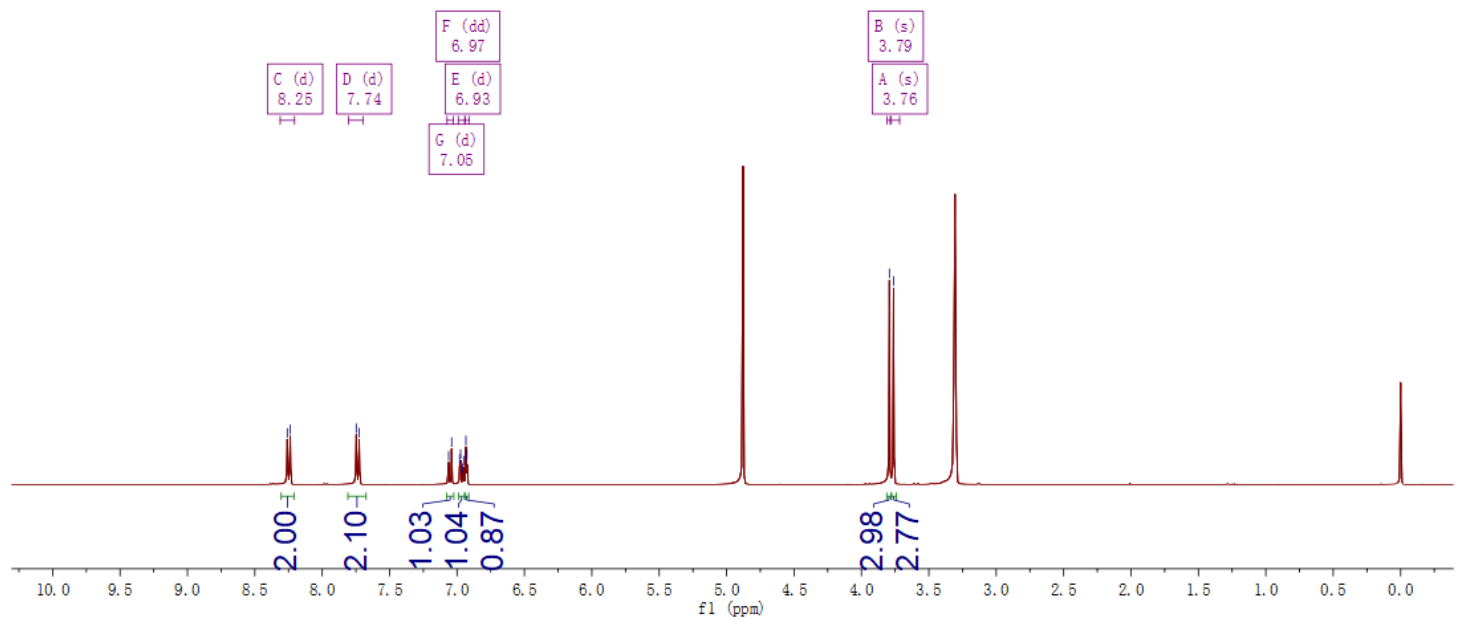



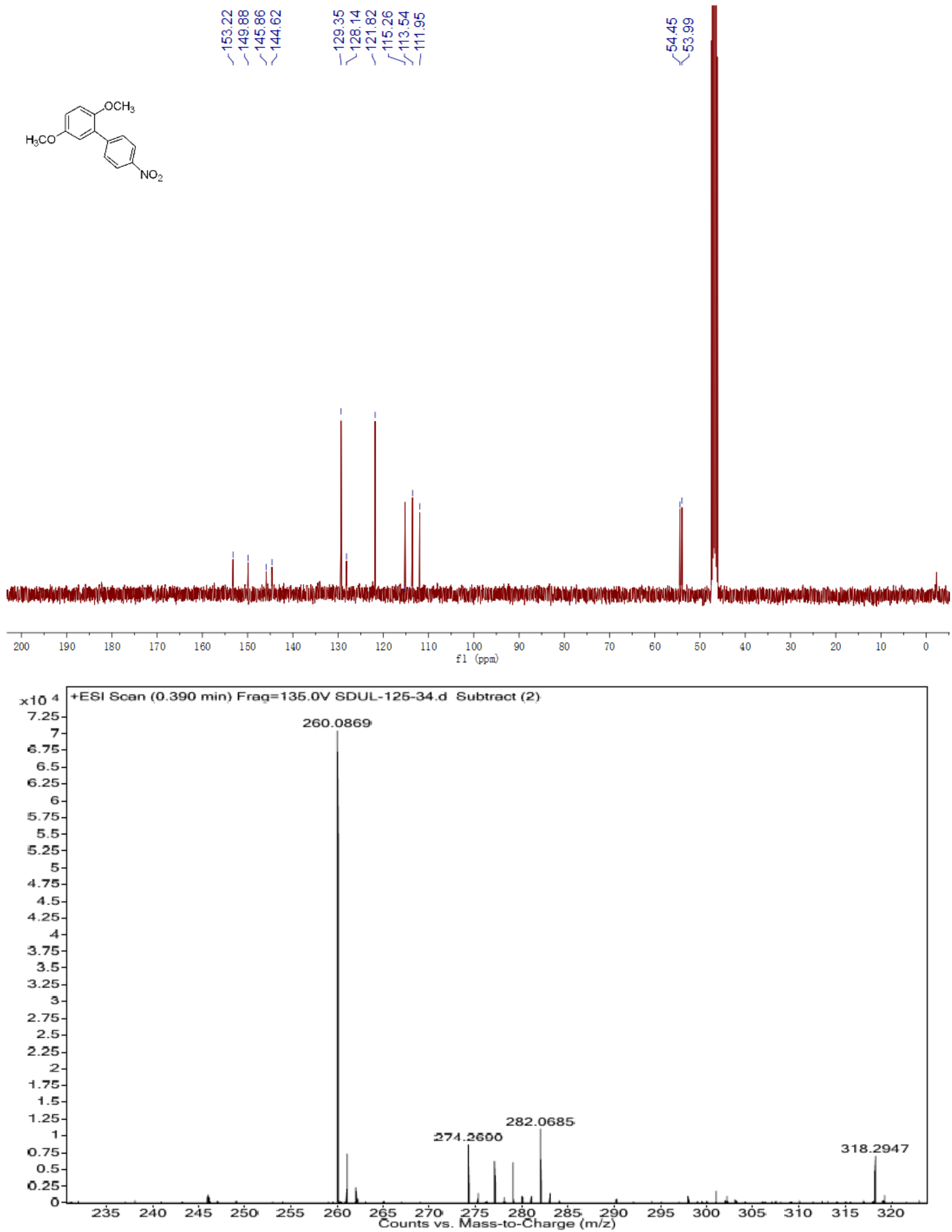

(E)-4-((2',5'-dimethoxy-[1,1'-biphenyl]-4-yl)diazenyl)pyridine (piCRAC-5) 


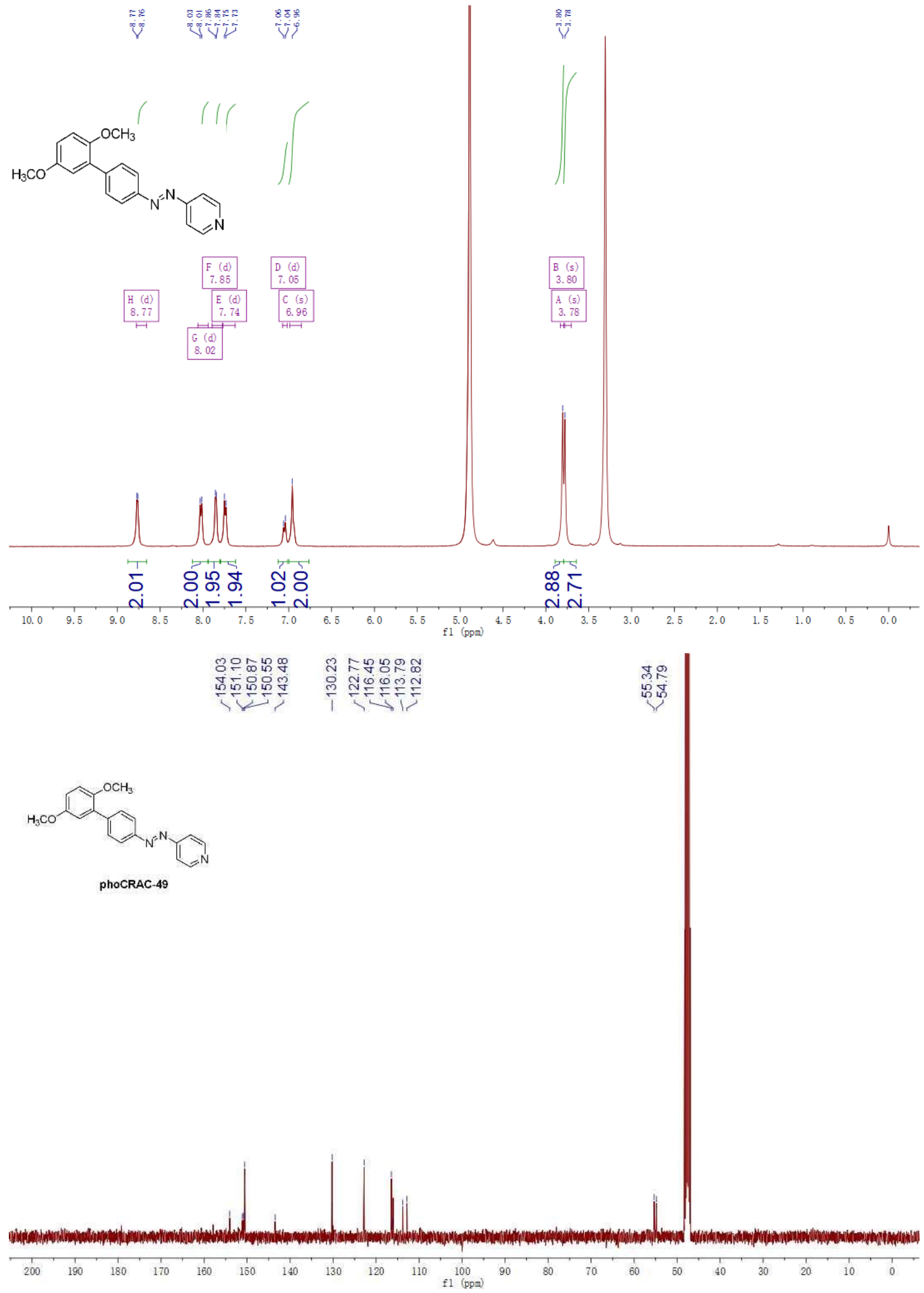



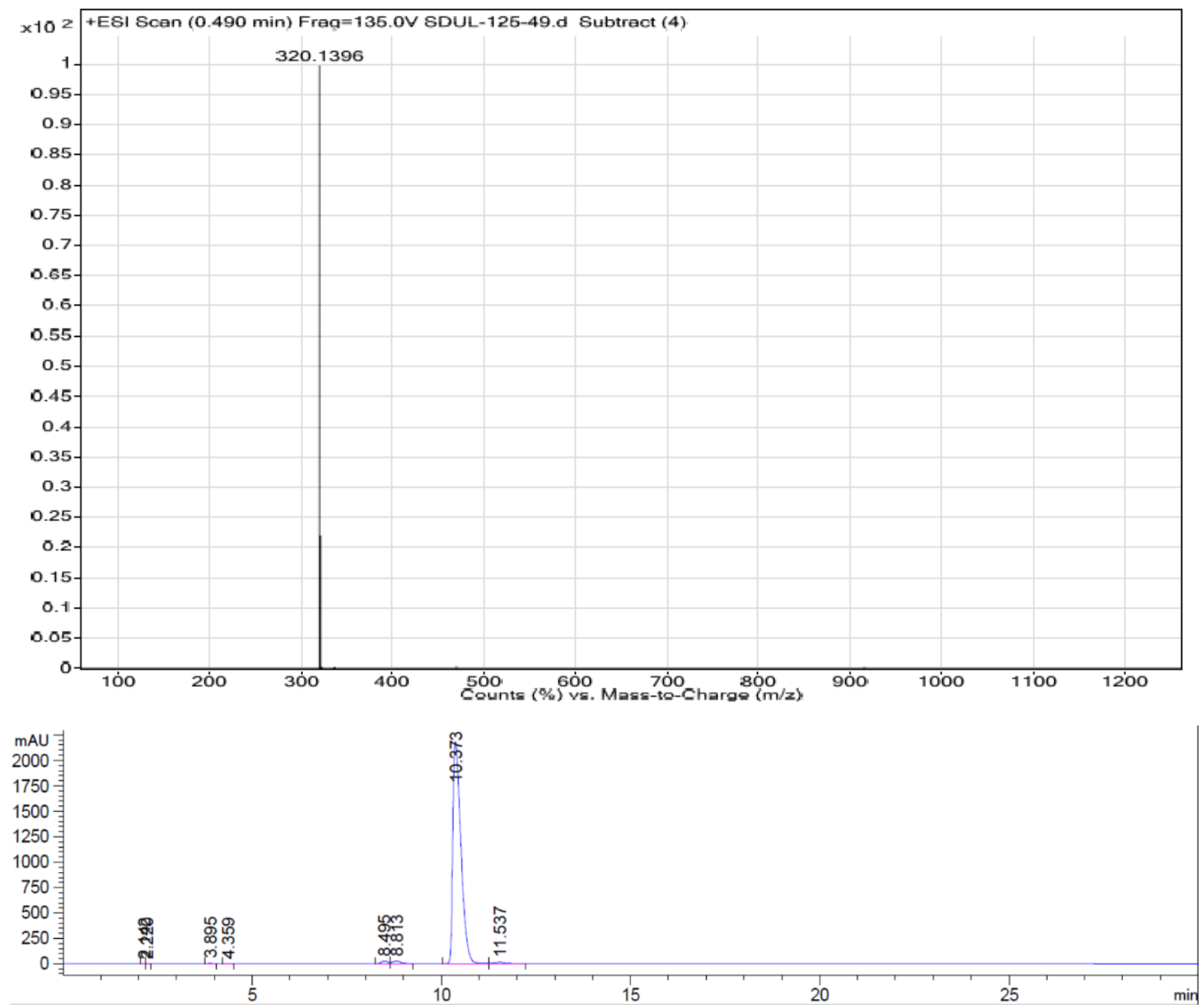

\section{2,6-difluoro-N-(1H-pyrazol-3-yl)benzamide (A-2)}




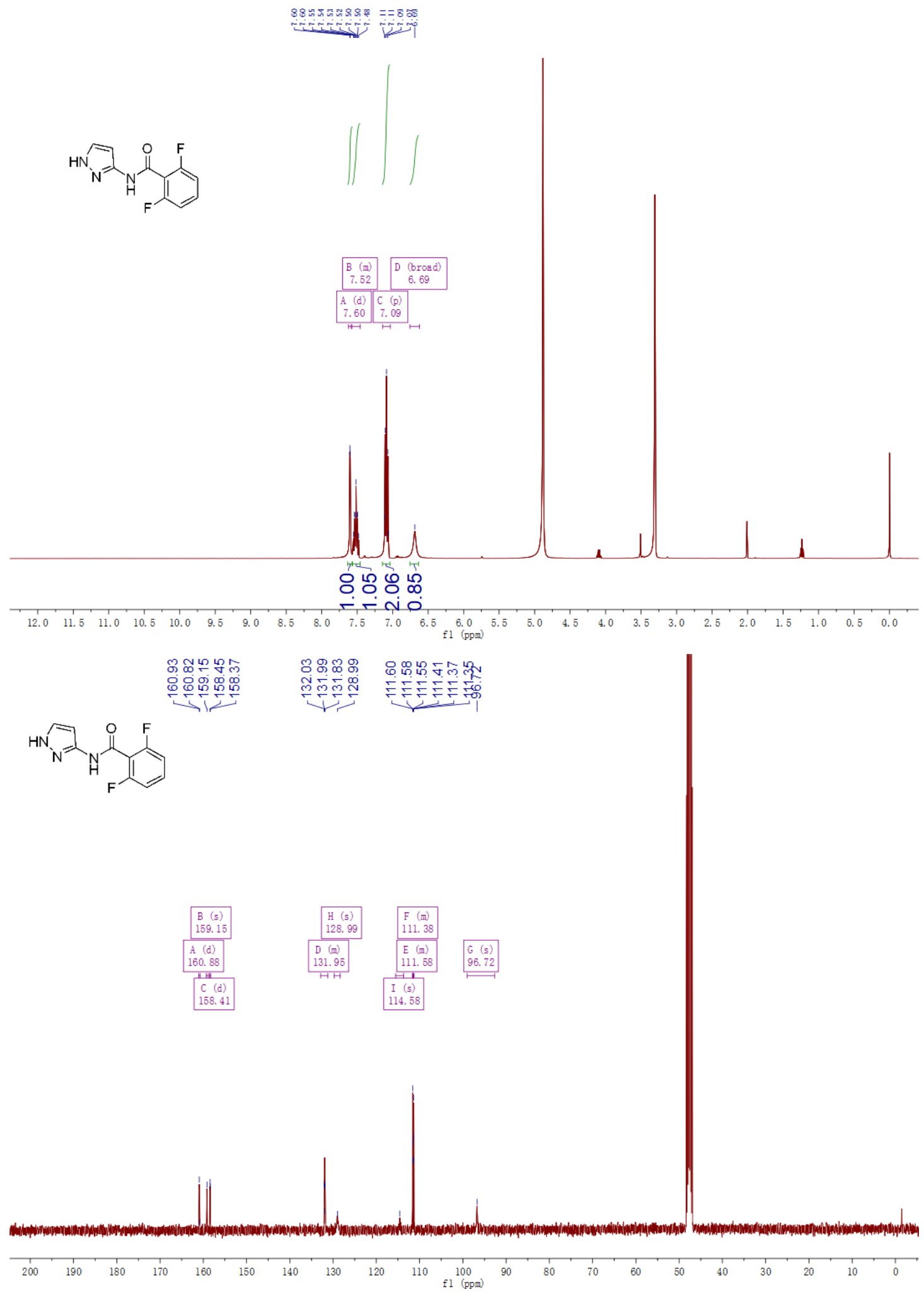




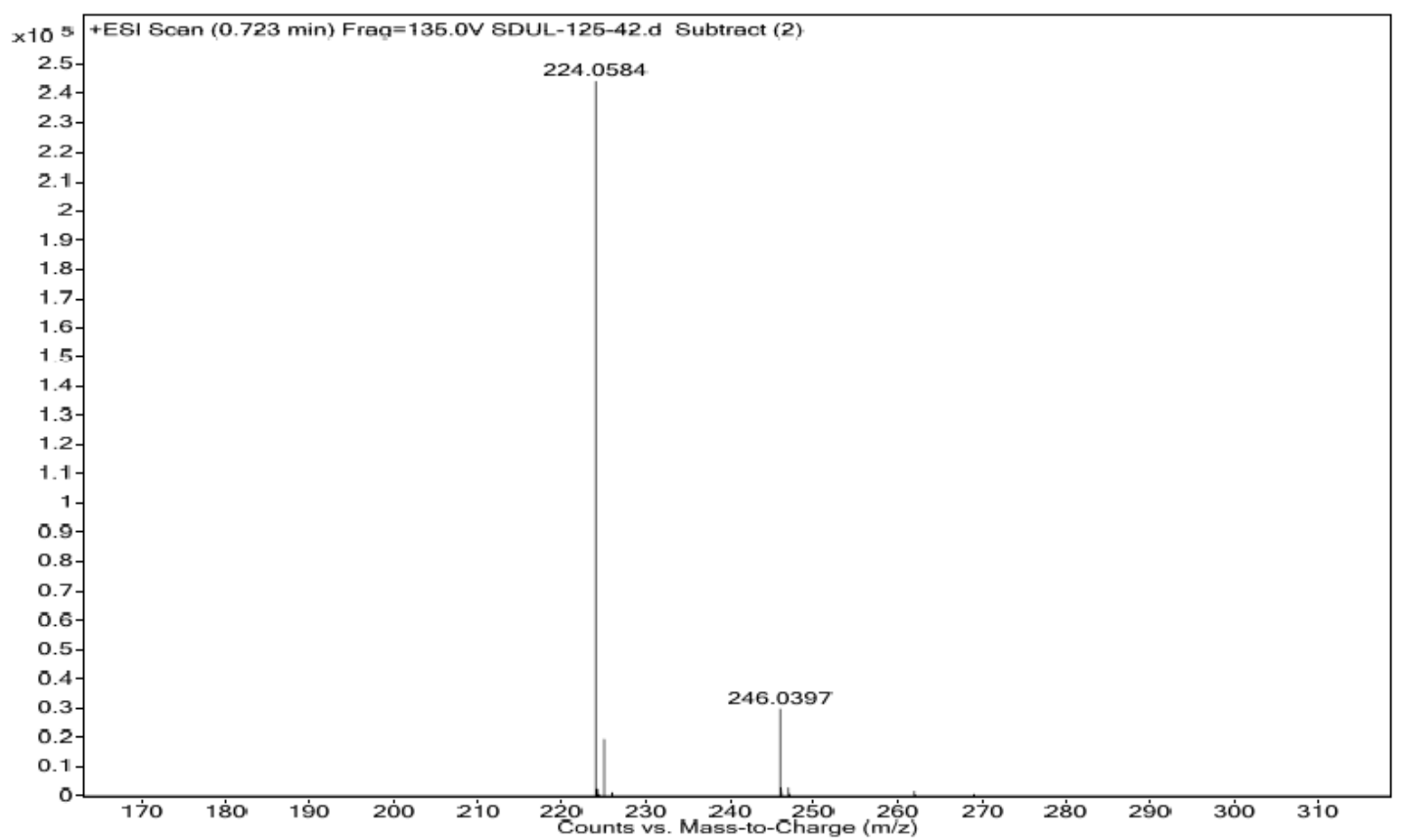

2,6-difluoro-N-(1-(2-fluoro-6-(trifluoromethyl)benzyl)-1H-pyrazol-3-yl)benzamide

(GSK5498A) 


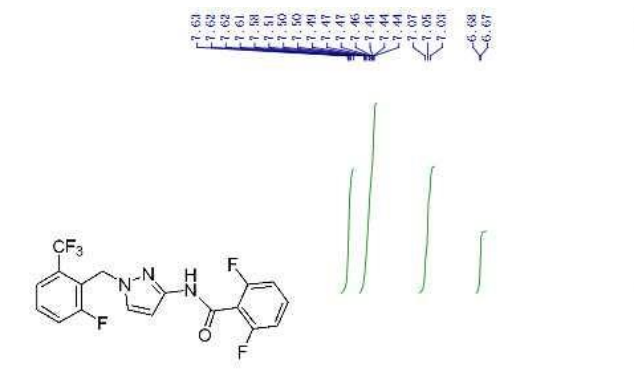

i

GSK-5498A

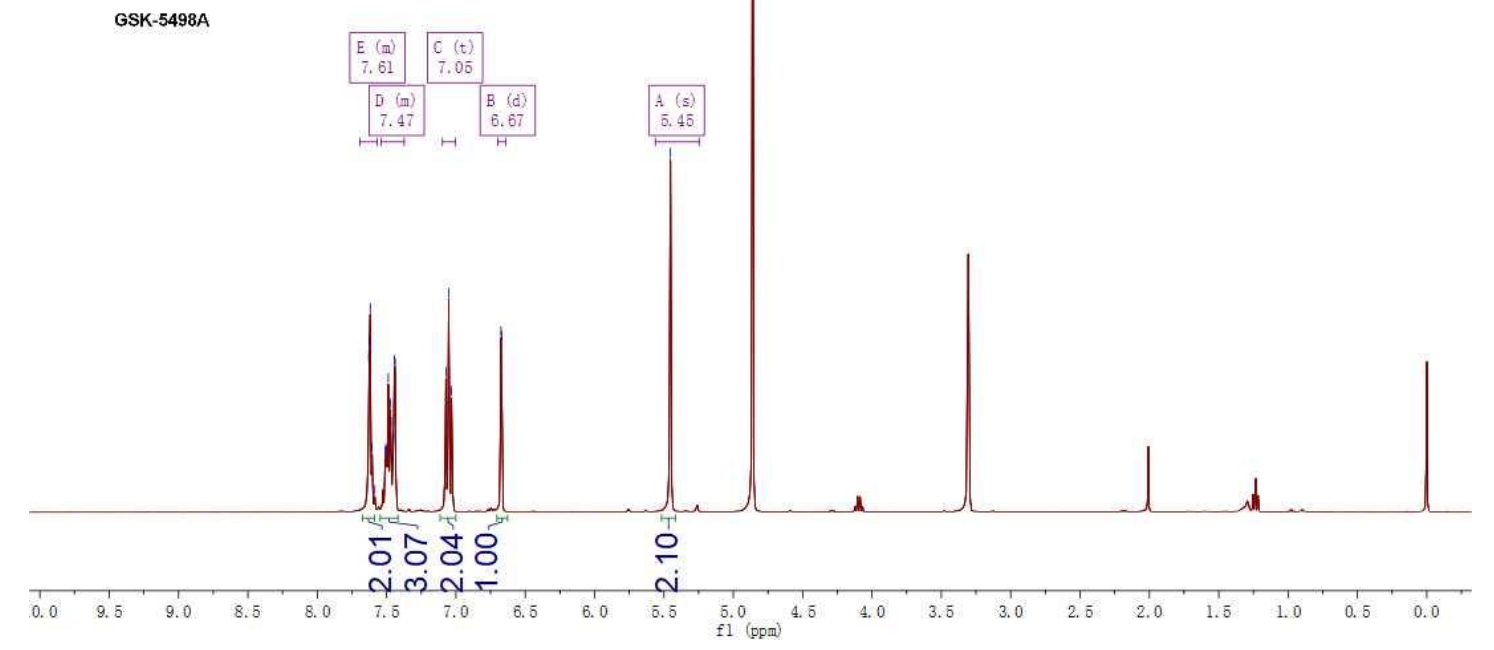

꾸유.

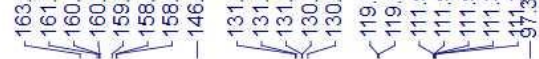

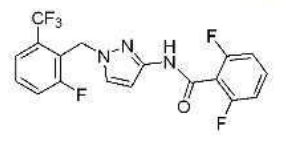

GSK-5498A

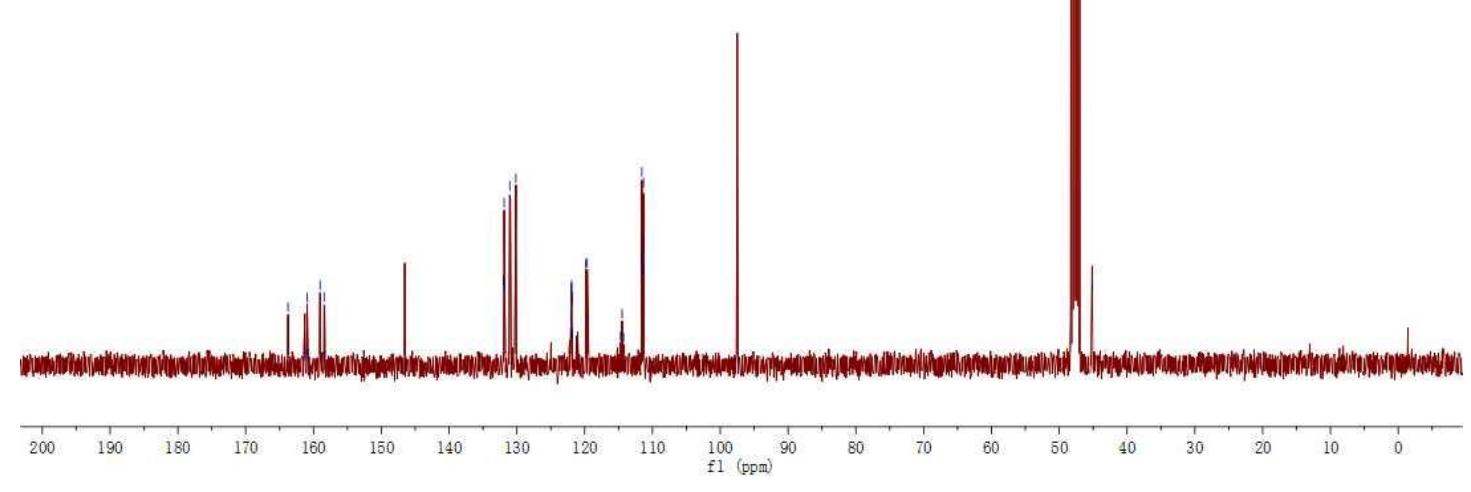



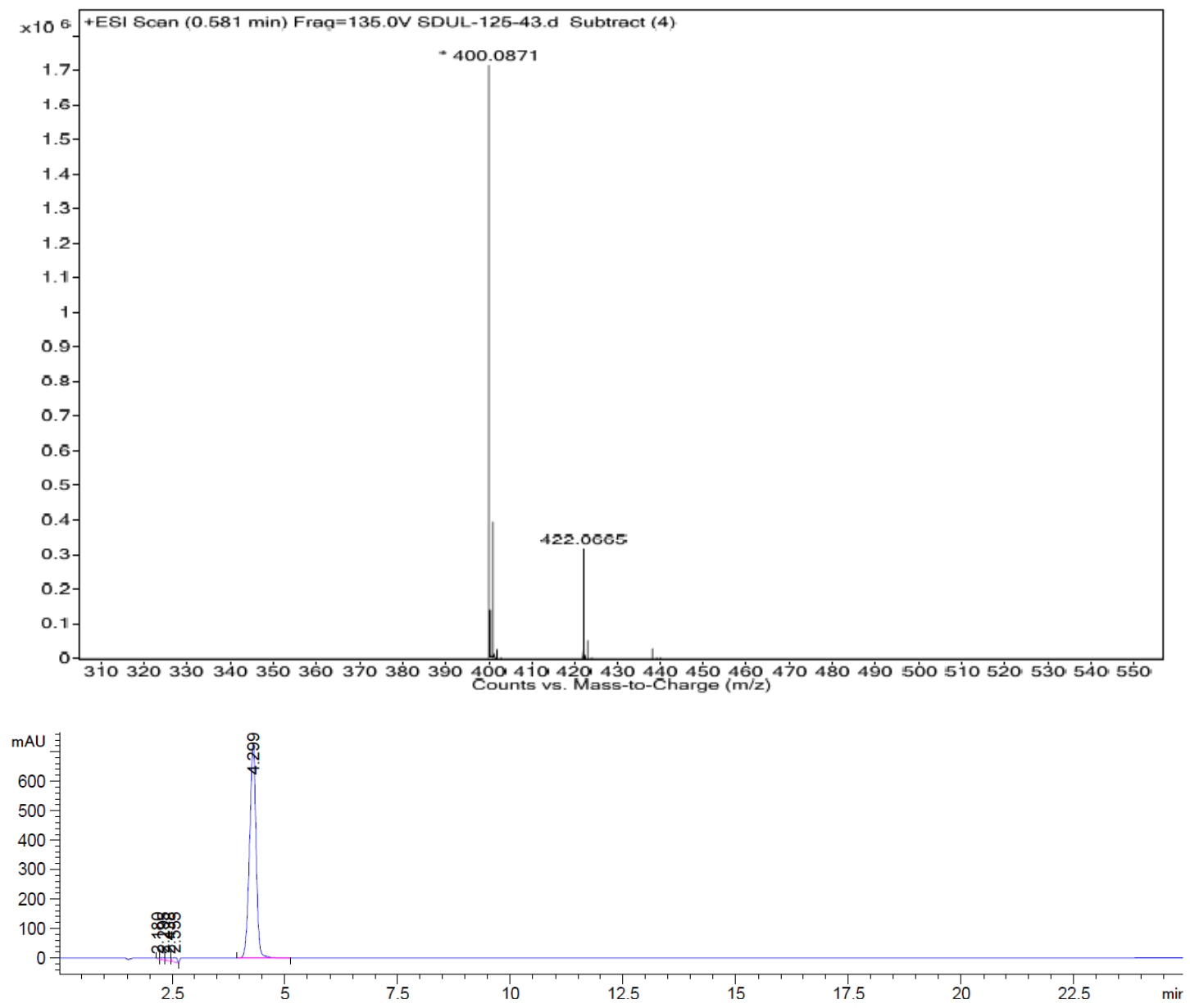

(E)-3-(phenyldiazenyl)-1H-pyrazole (6-3) 

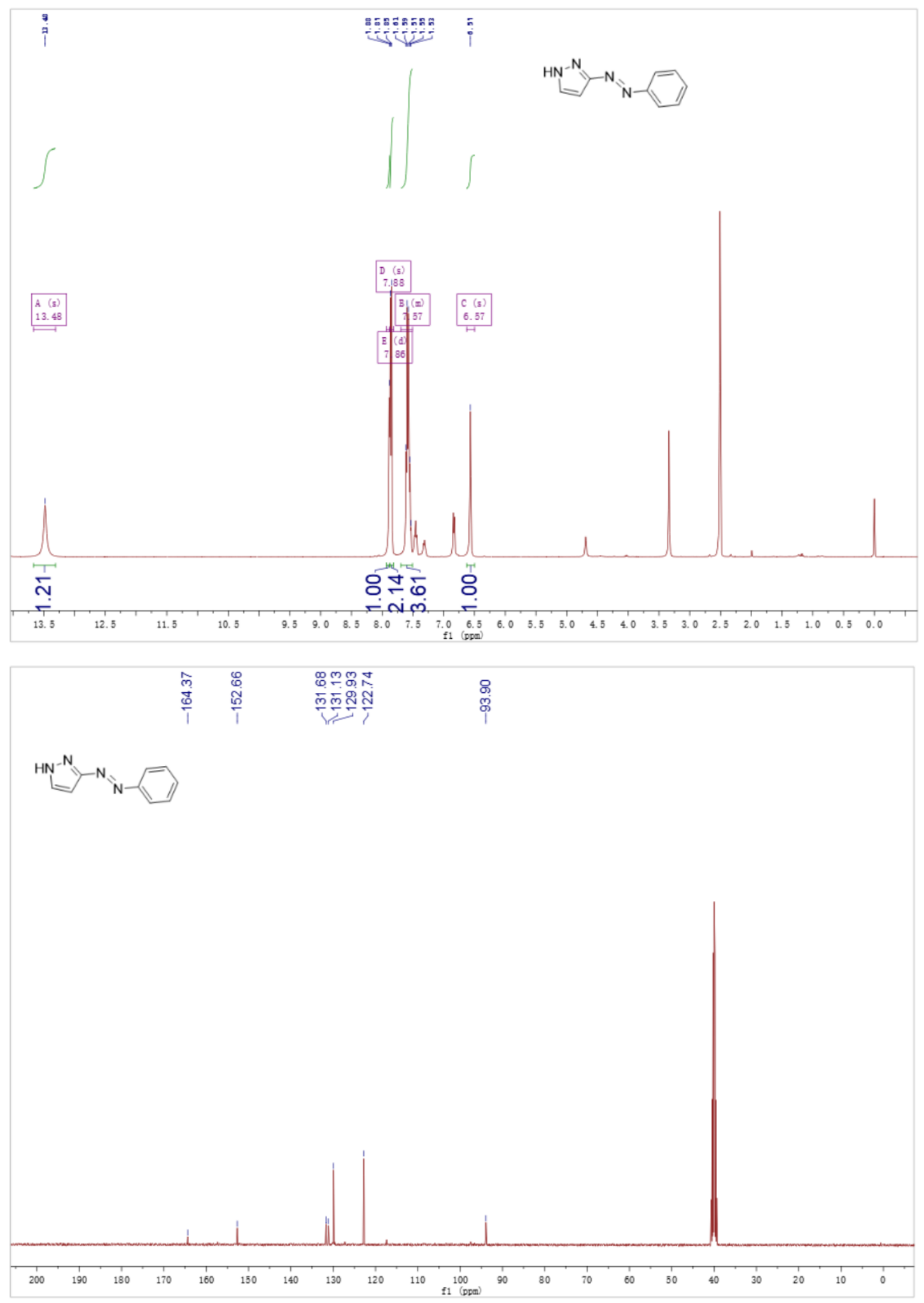

S64 


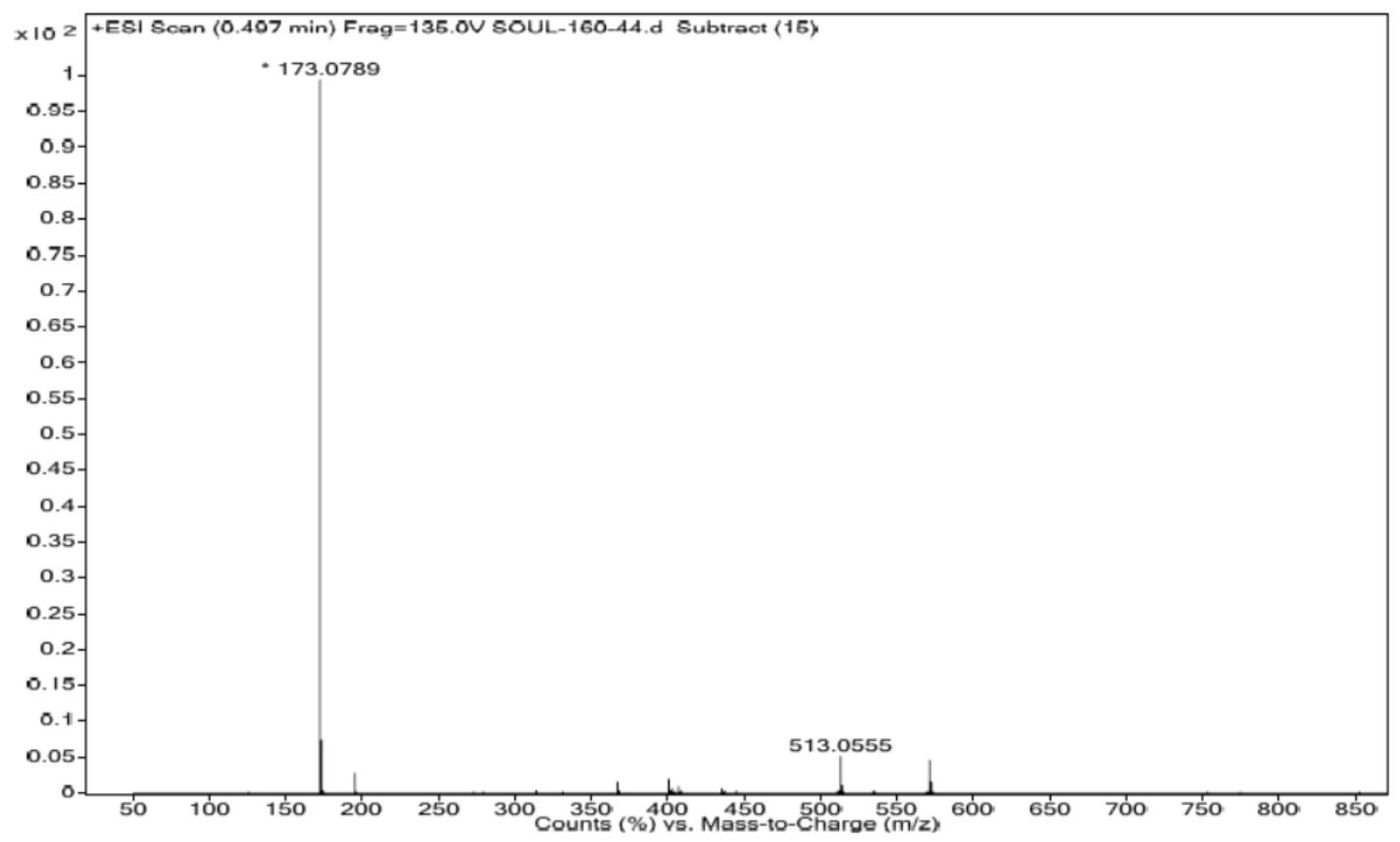

(E)-1-(2-fluoro-6-(trifluoromethyl)benzyl)-3-(phenyldiazenyl)-1H-pyrazole (piCRAC-6)

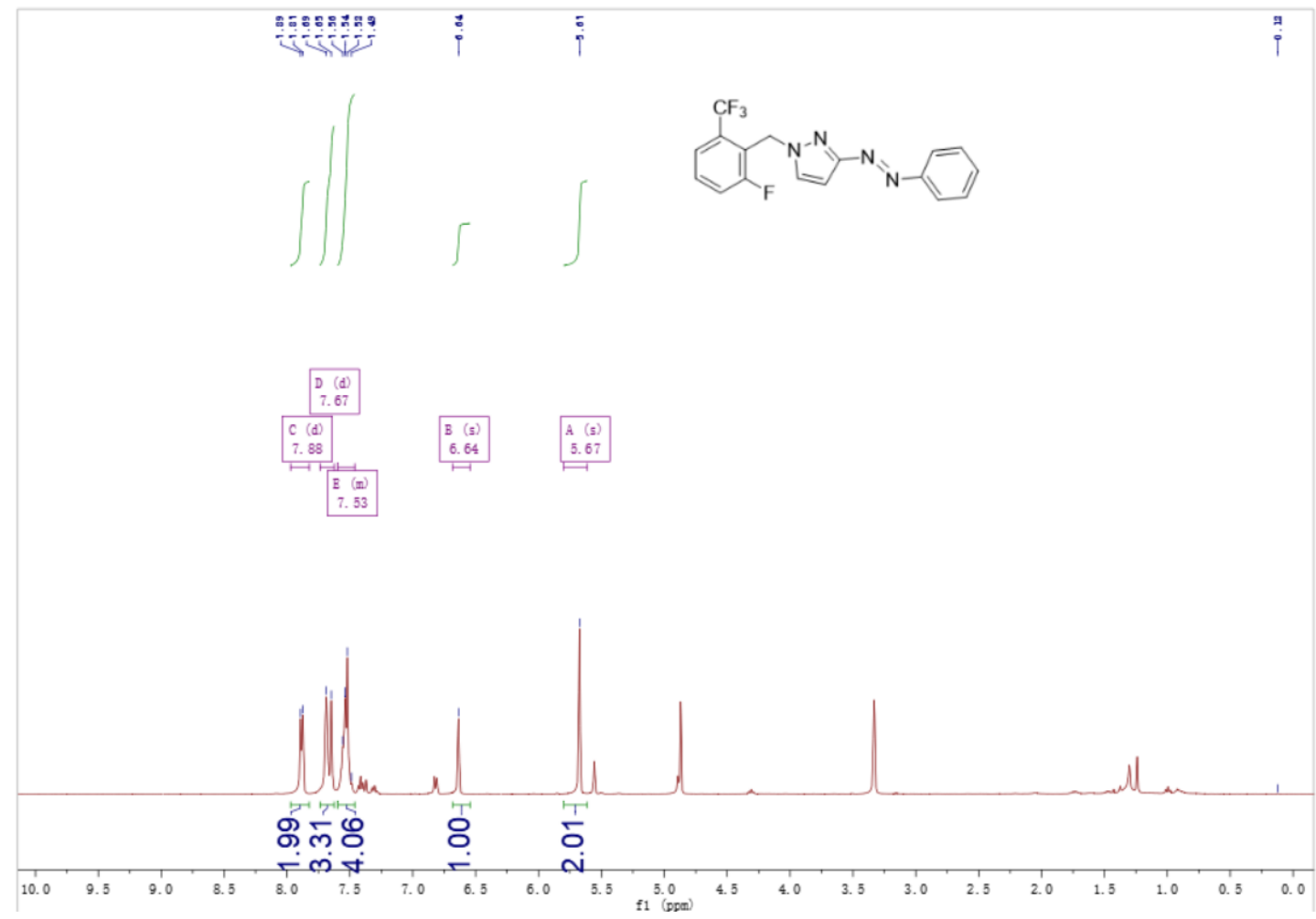



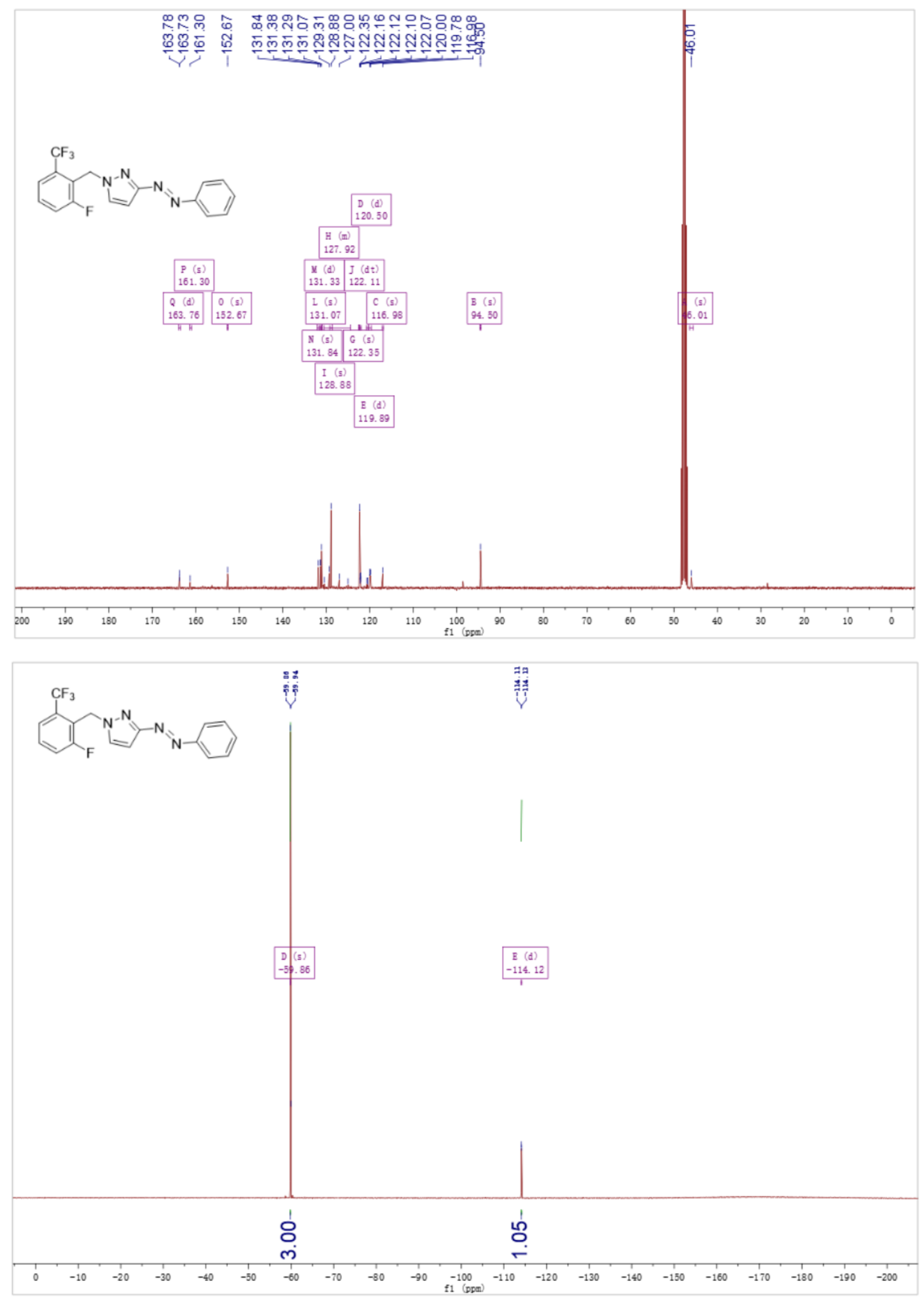

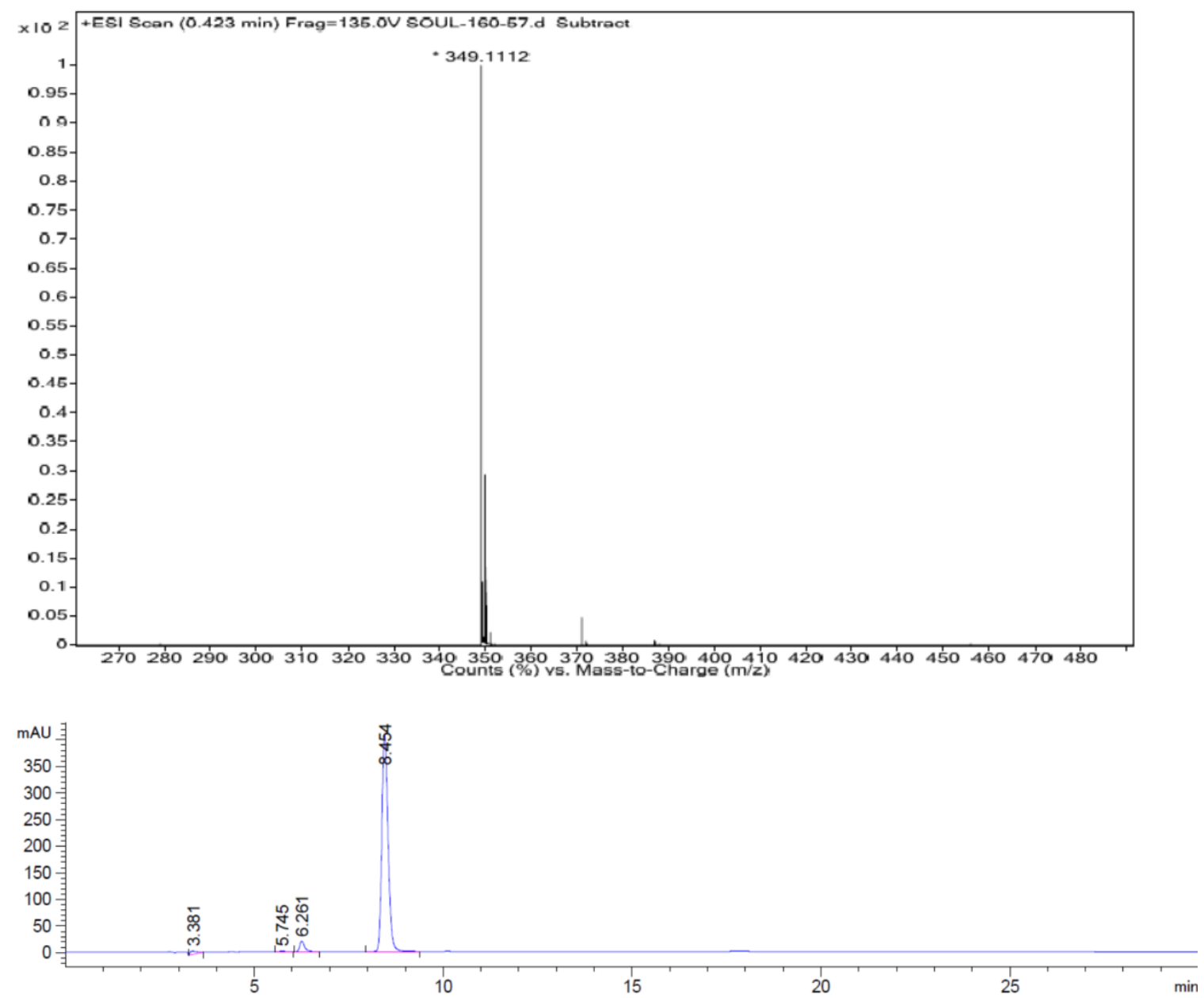

methyl 3,5-difluoro-4-nitrosobenzoate (7-2) 

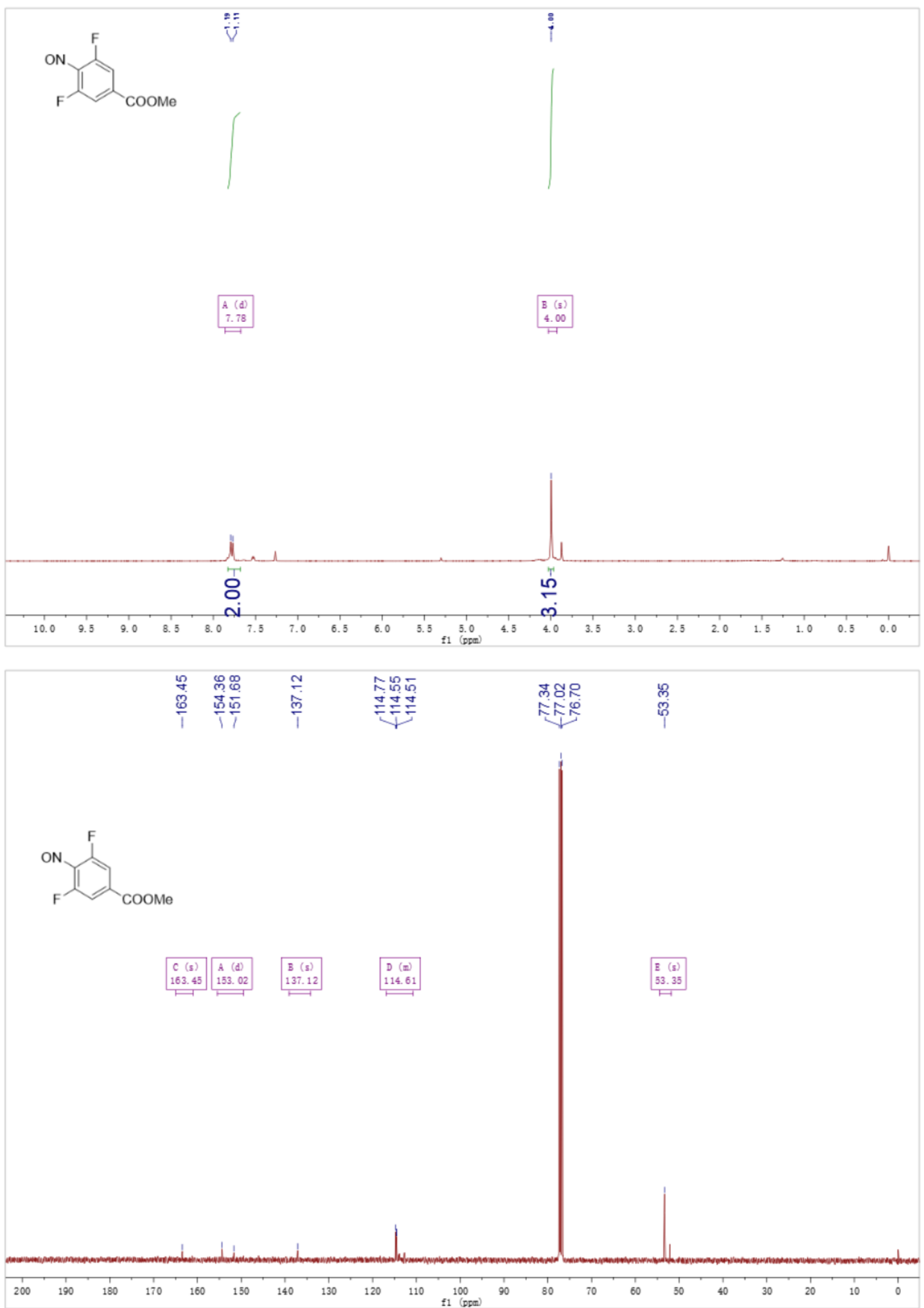


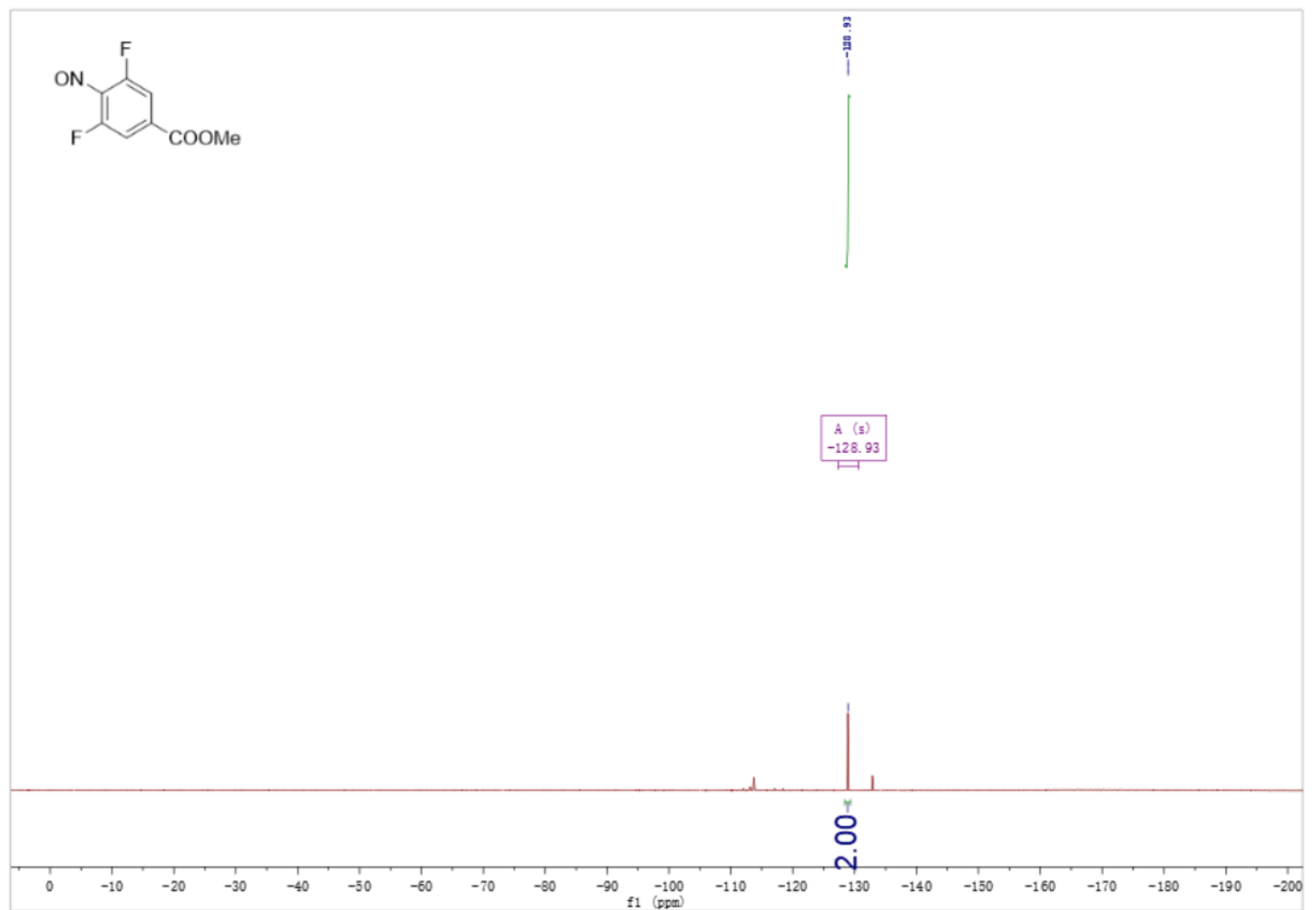

methyl (E)-4-((1H-pyrazol-3-yl)diazenyl)-3,5-difluorobenzoate (7-3)

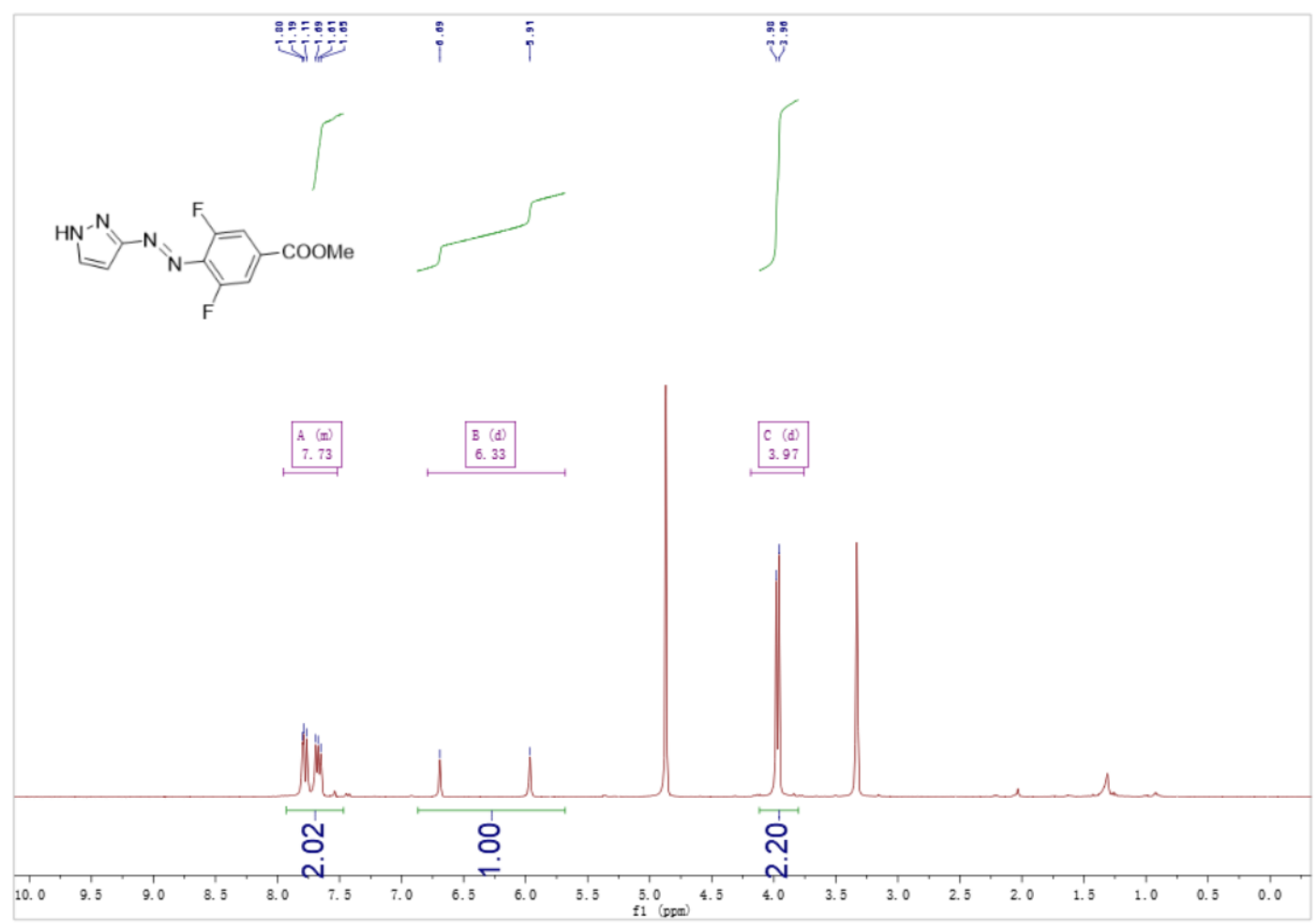



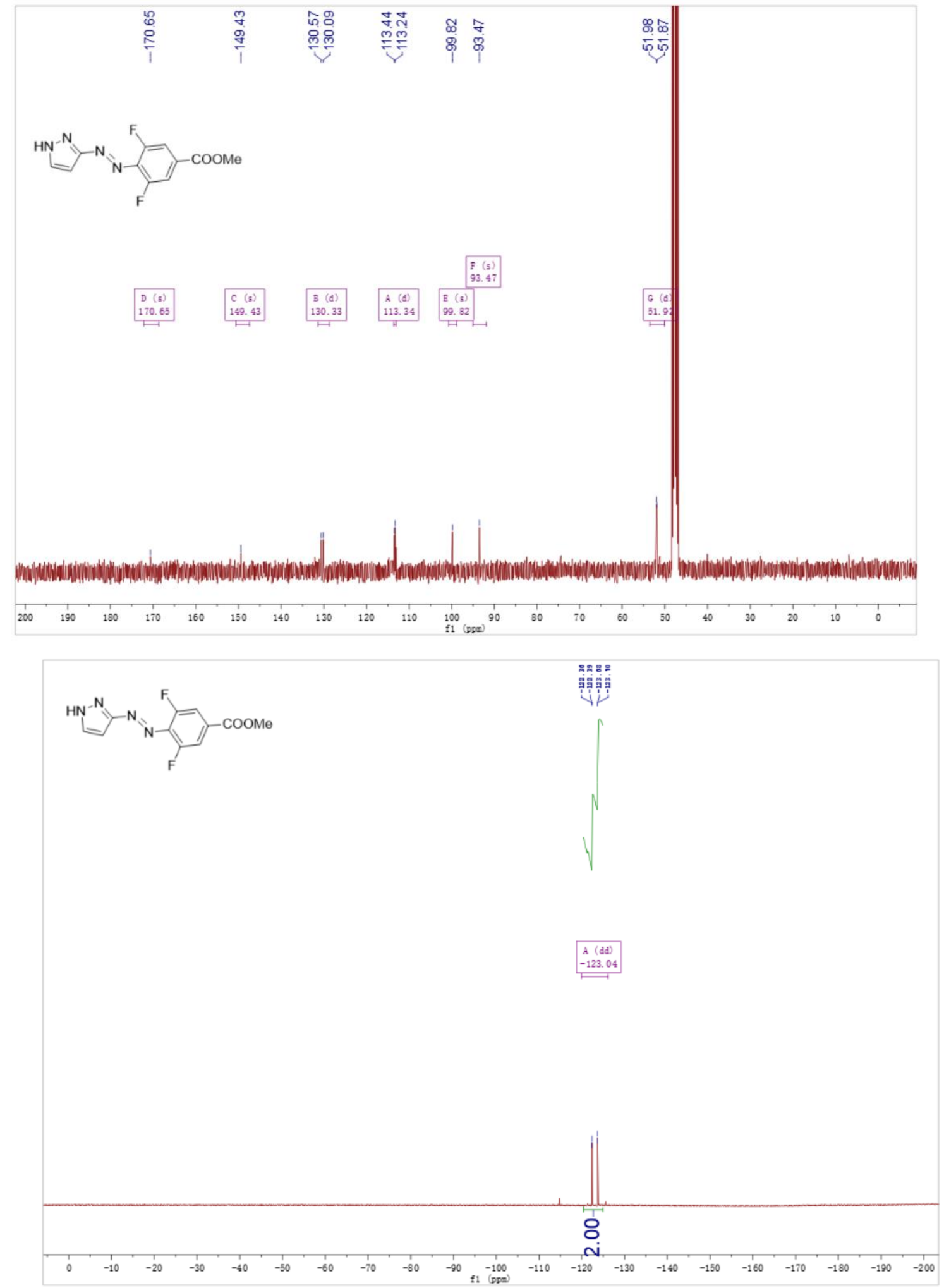


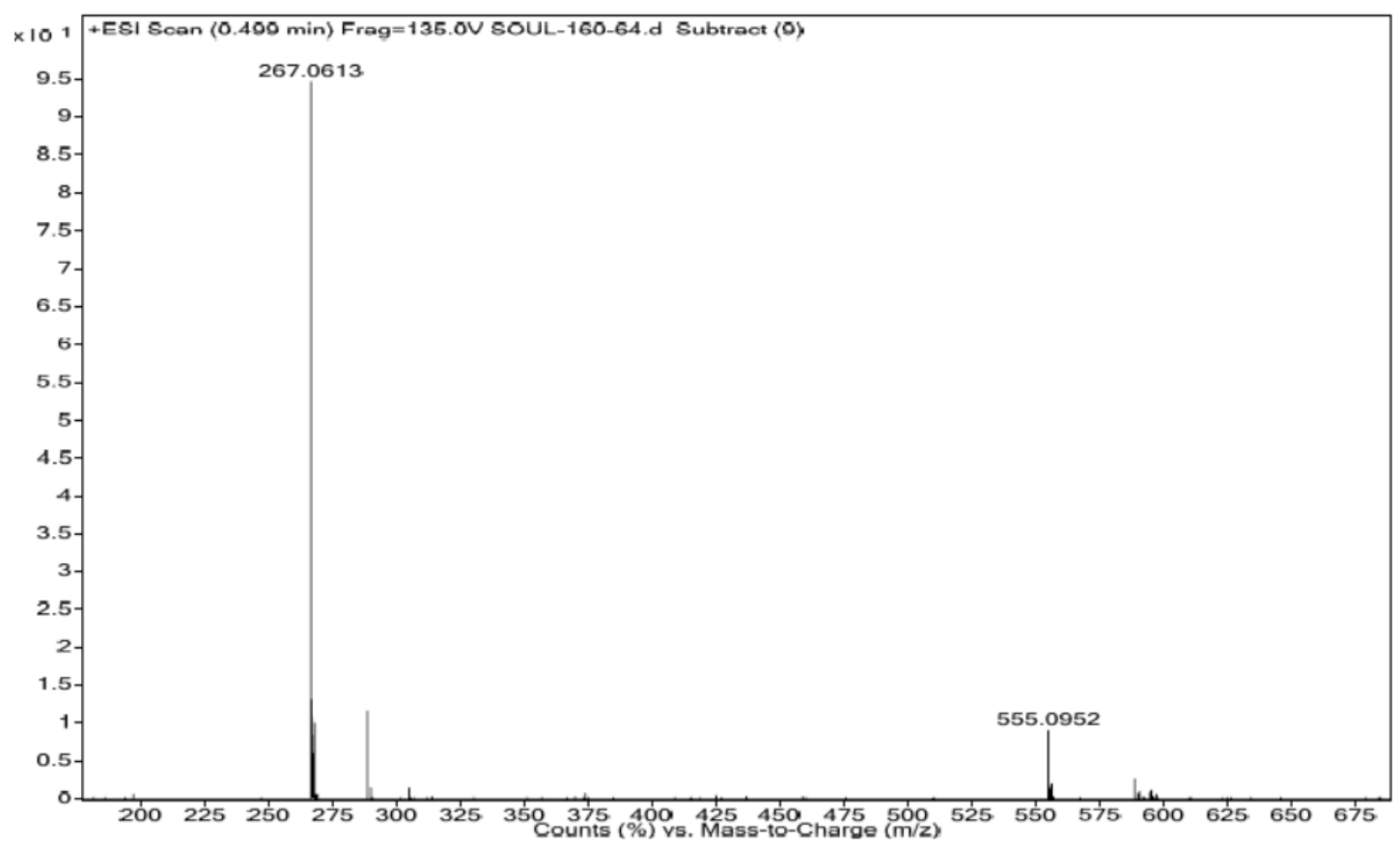

methyl (E)-3,5-difluoro-4-((1-(2-fluoro-6-(trifluoromethyl)benzyl)-1H-pyrazol-3-yl)diazenyl)benzoate (piCRAC-7)

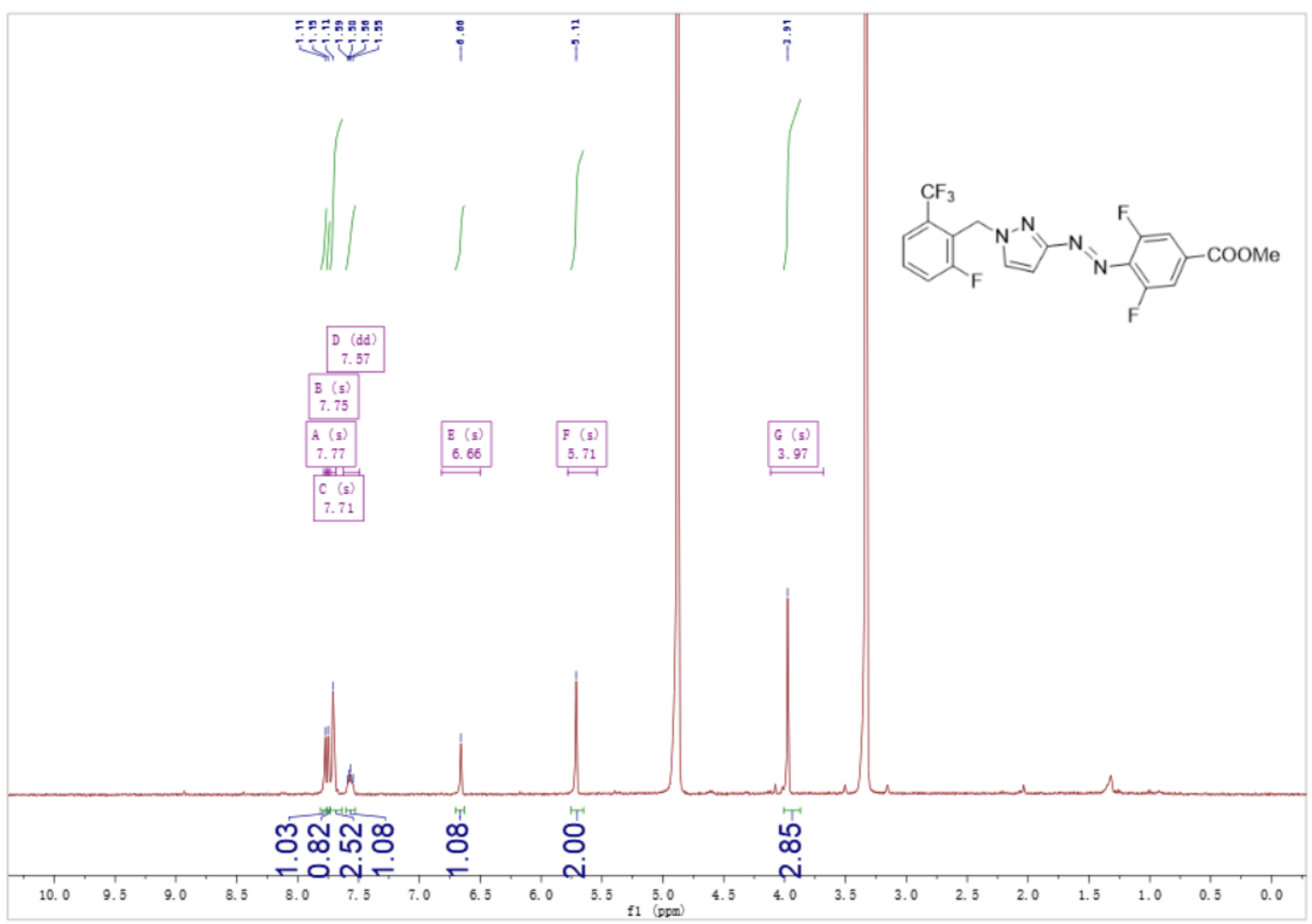



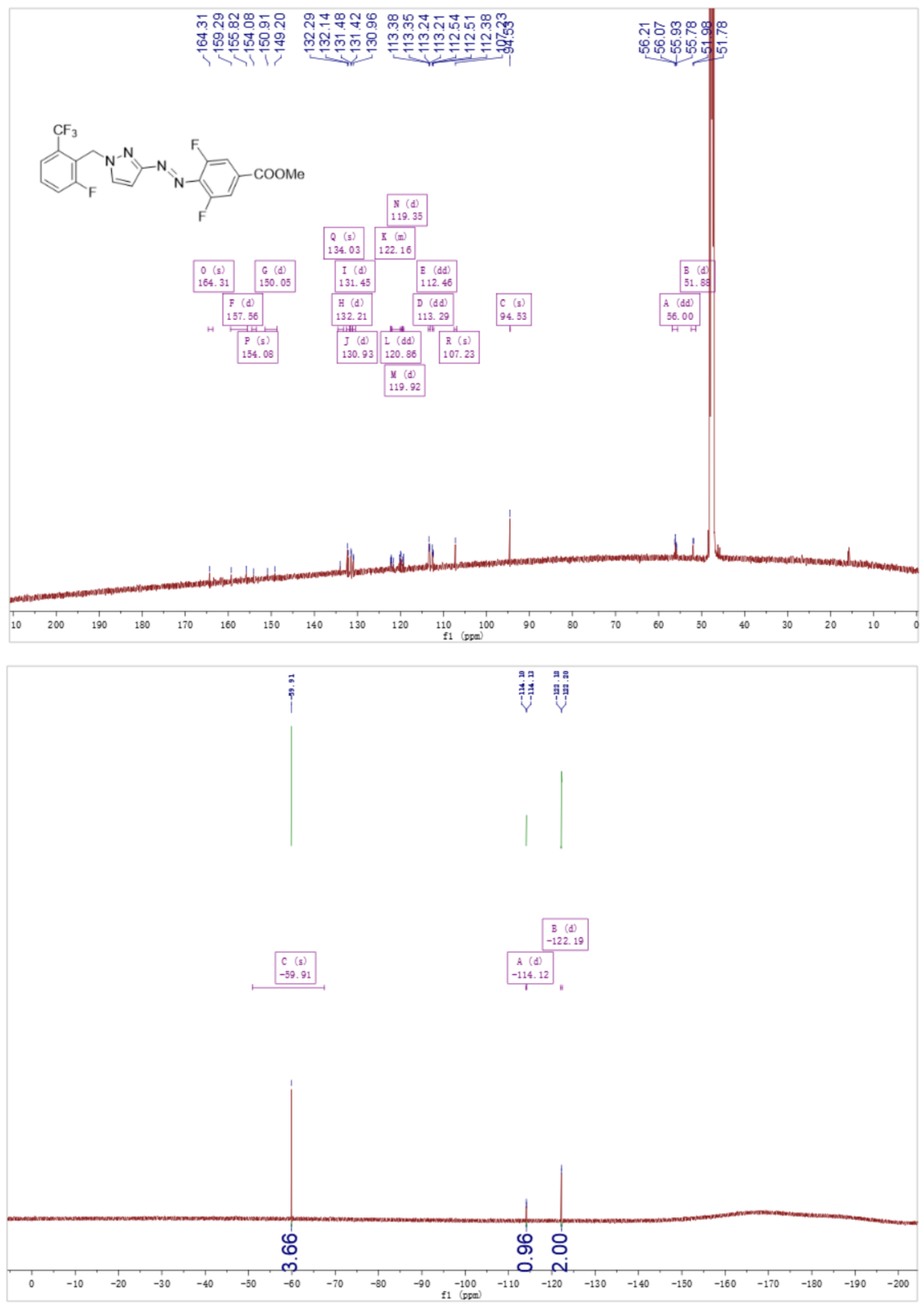

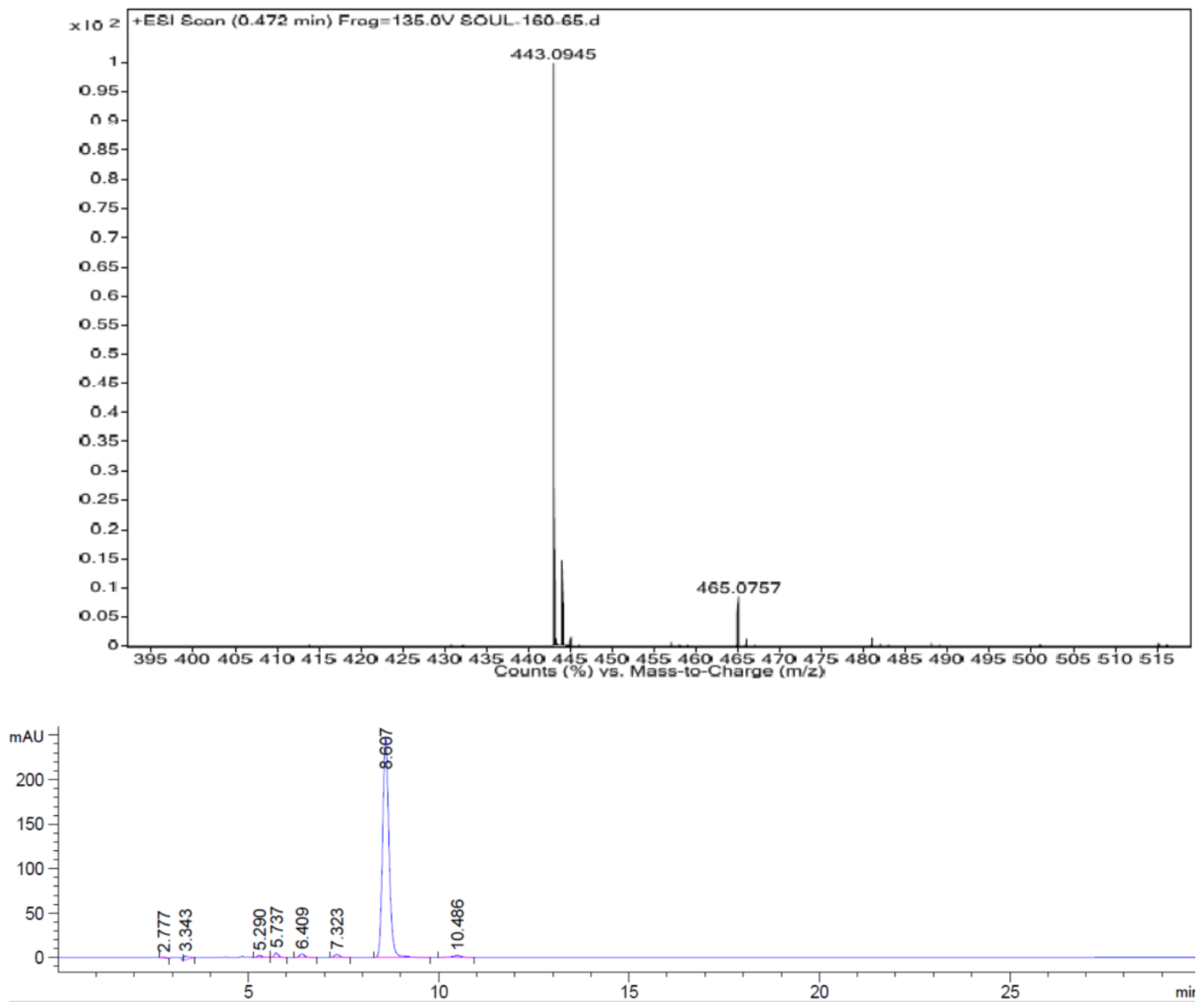

methyl 3,5-difluoro-4-nitrosobenzoate (8-2) 

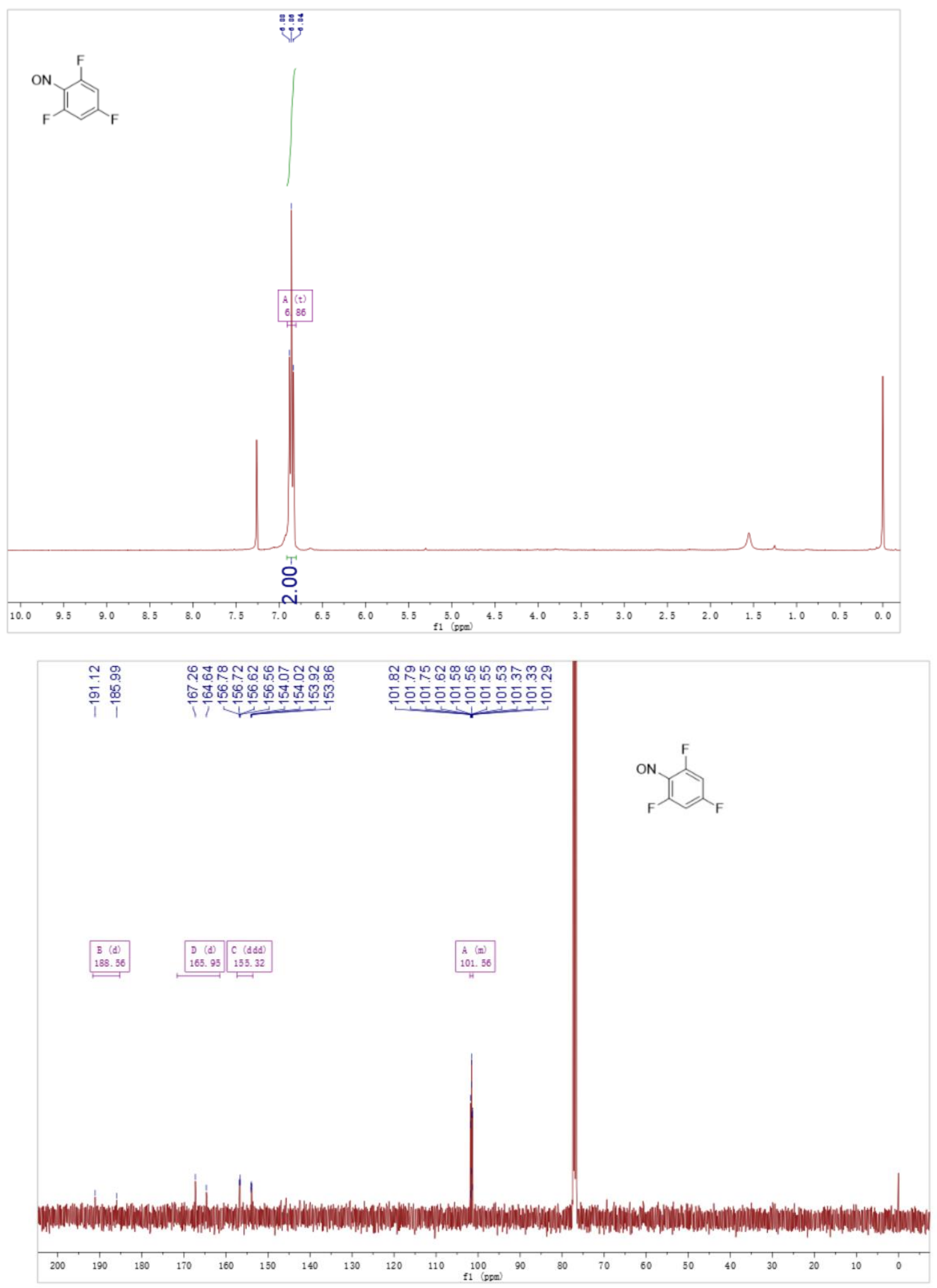


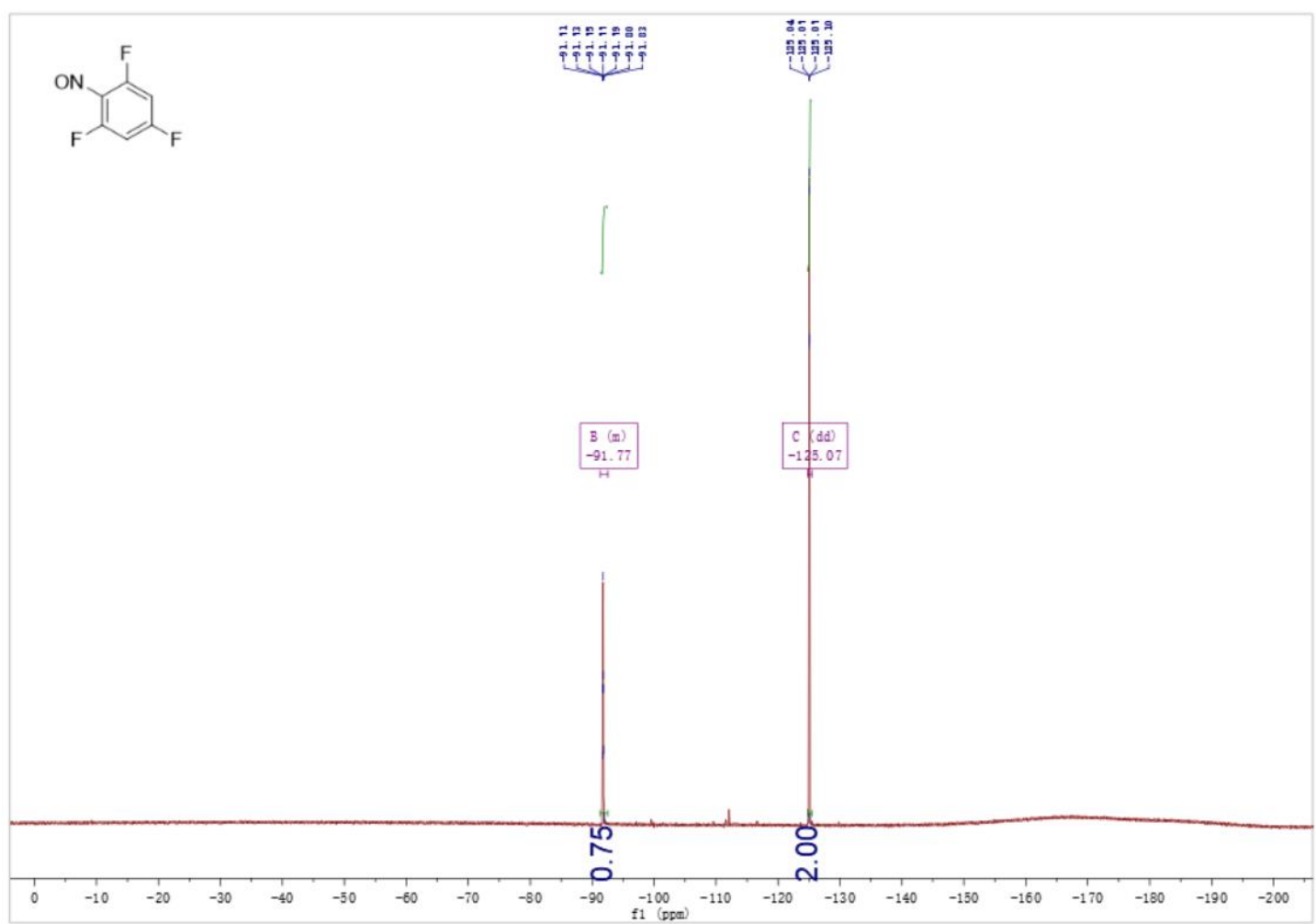

(E)-3-((2,4,6-trifluorophenyl)diazenyl)-1H-pyrazole (8-3)

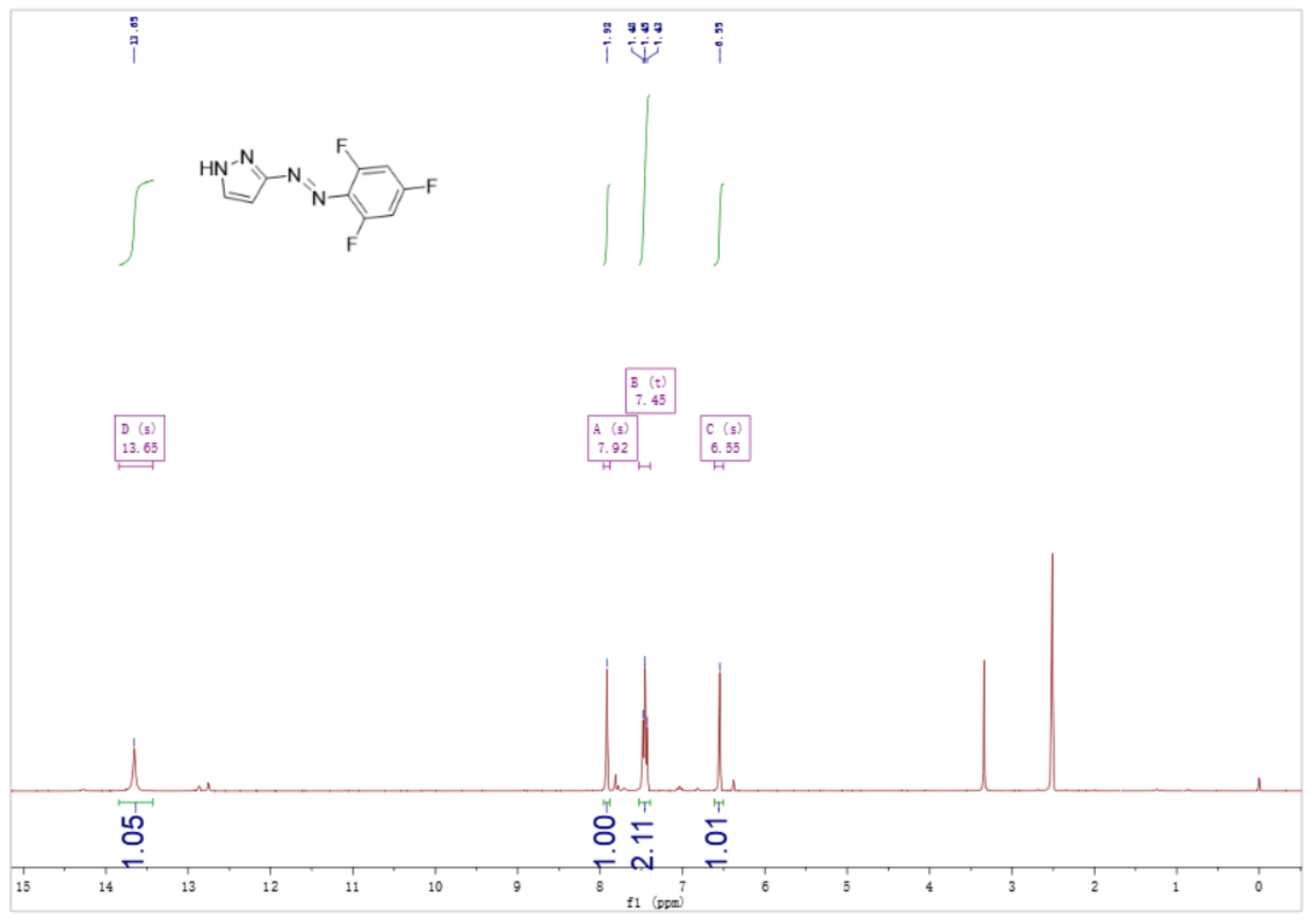



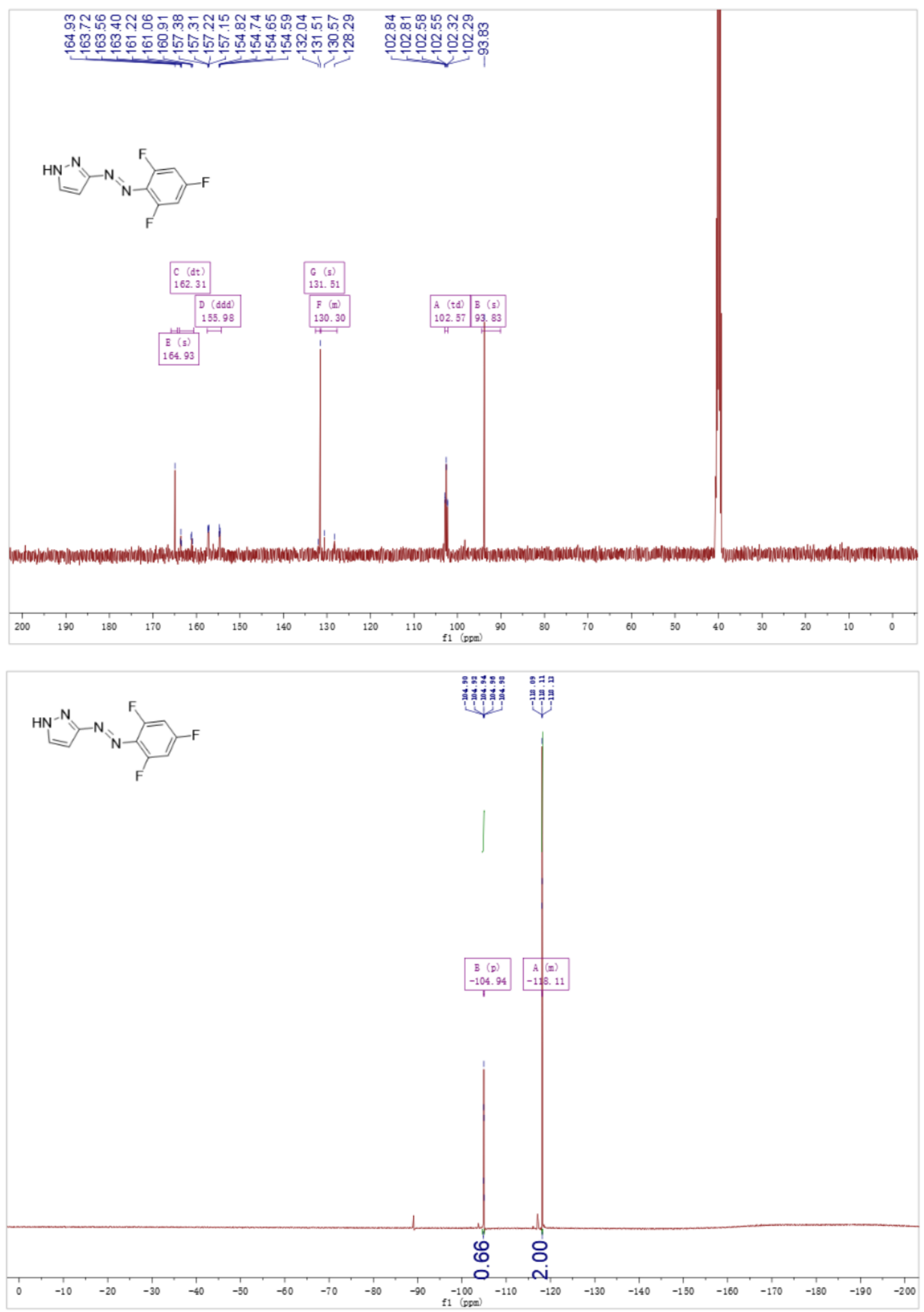


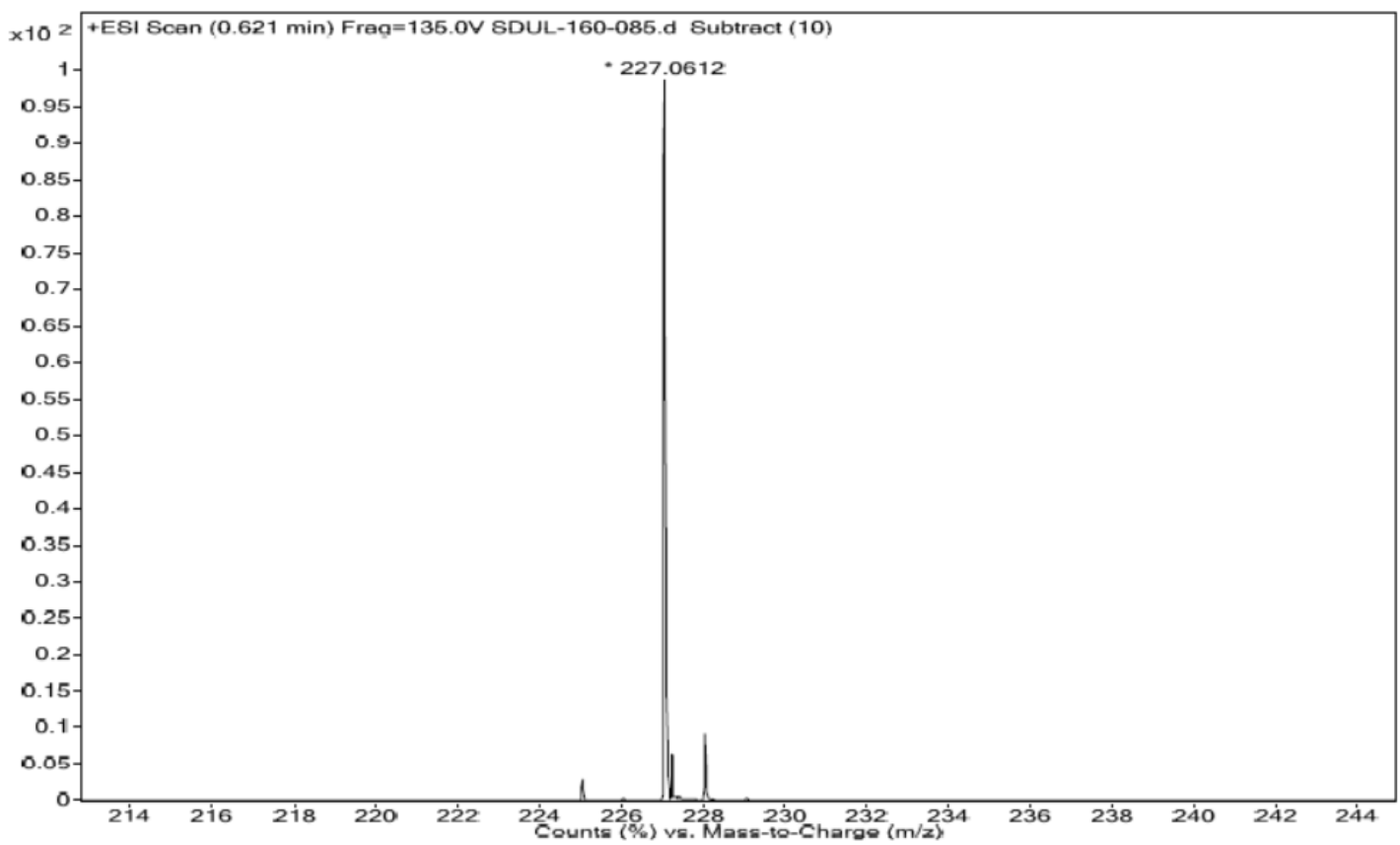

(E)-1-(2-fluoro-6-(trifluoromethyl)benzyl)-3-((2,4,6-trifluorophenyl)diazenyl)-1H-pyrazole (piCRAC-8)

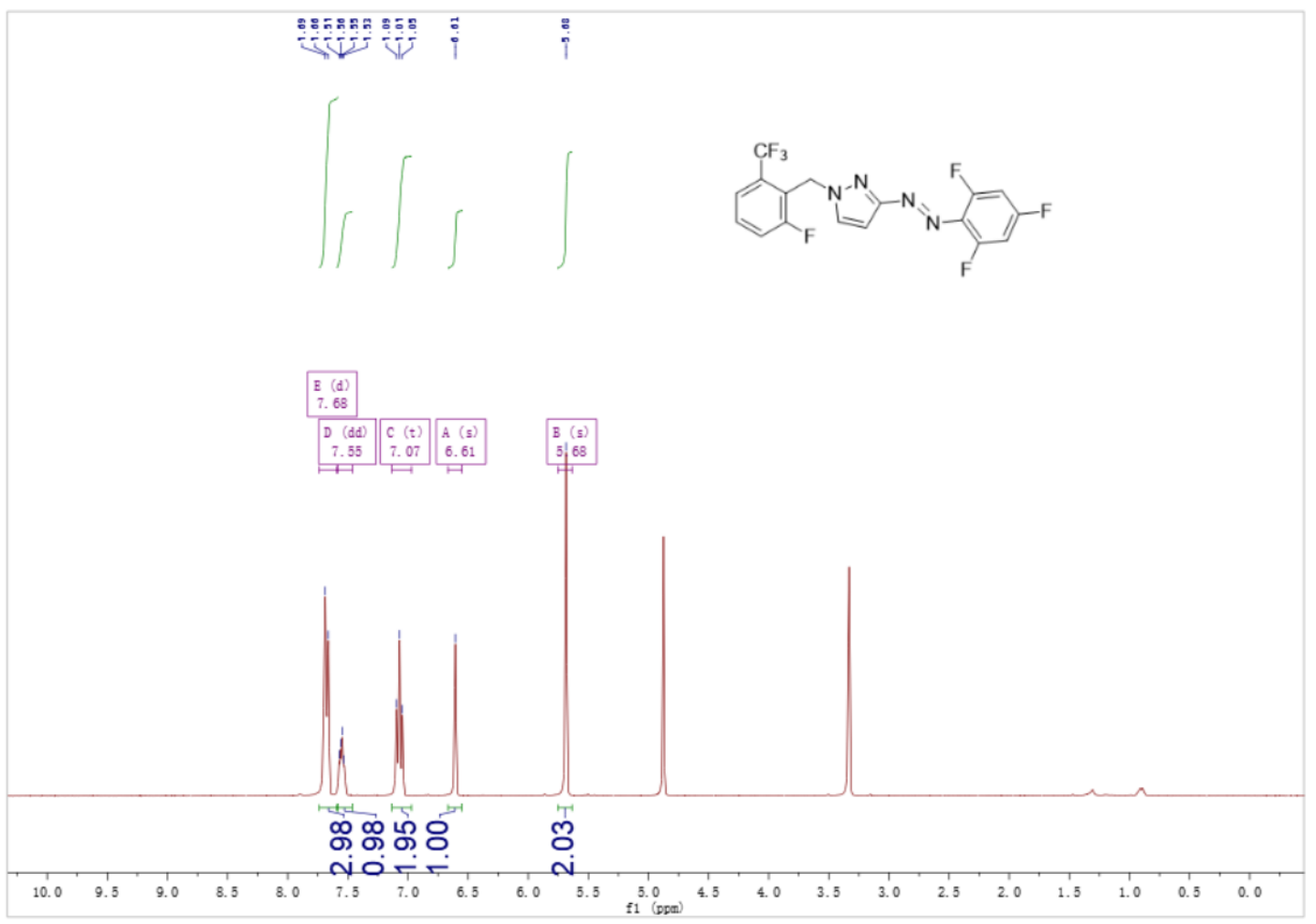



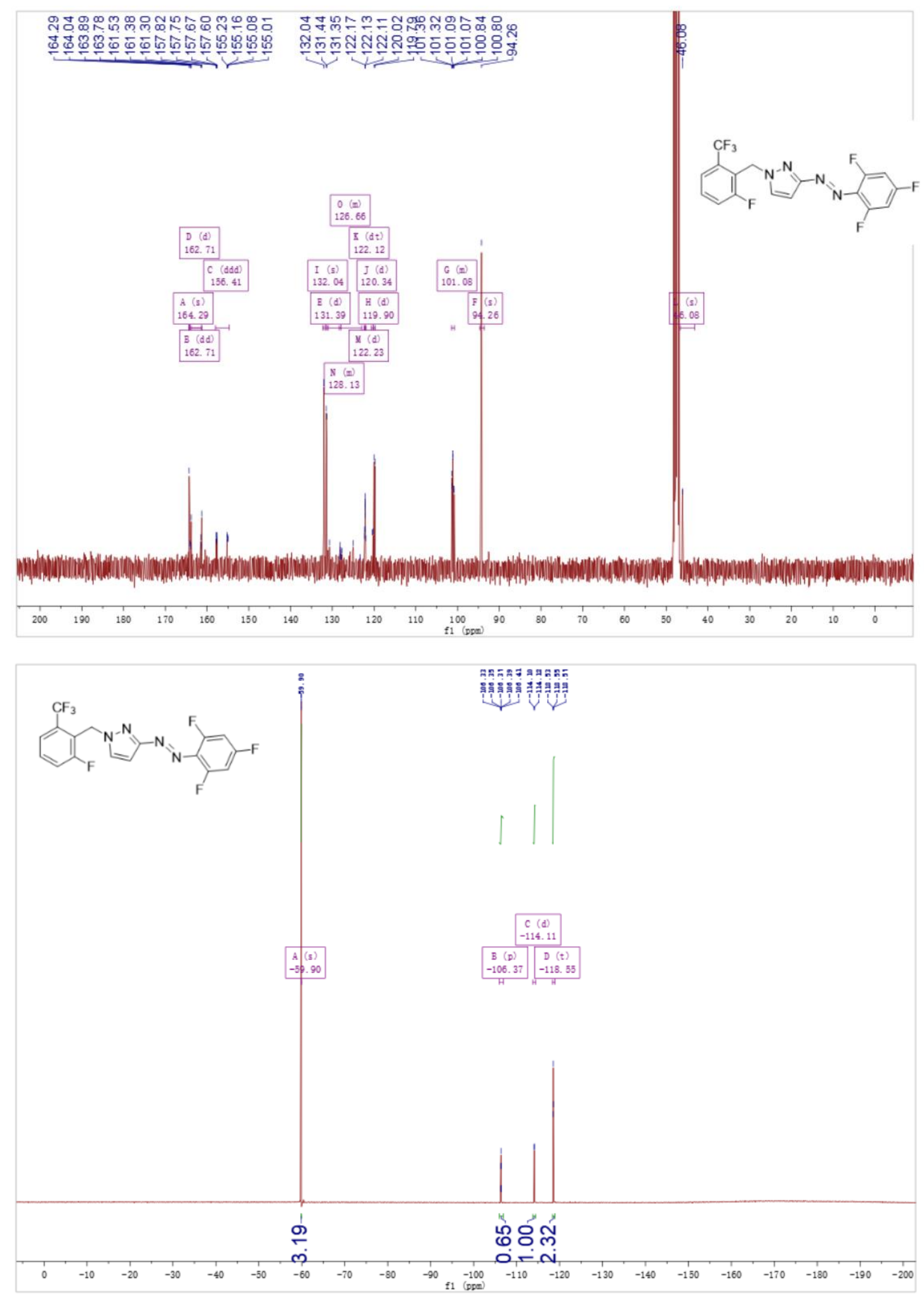

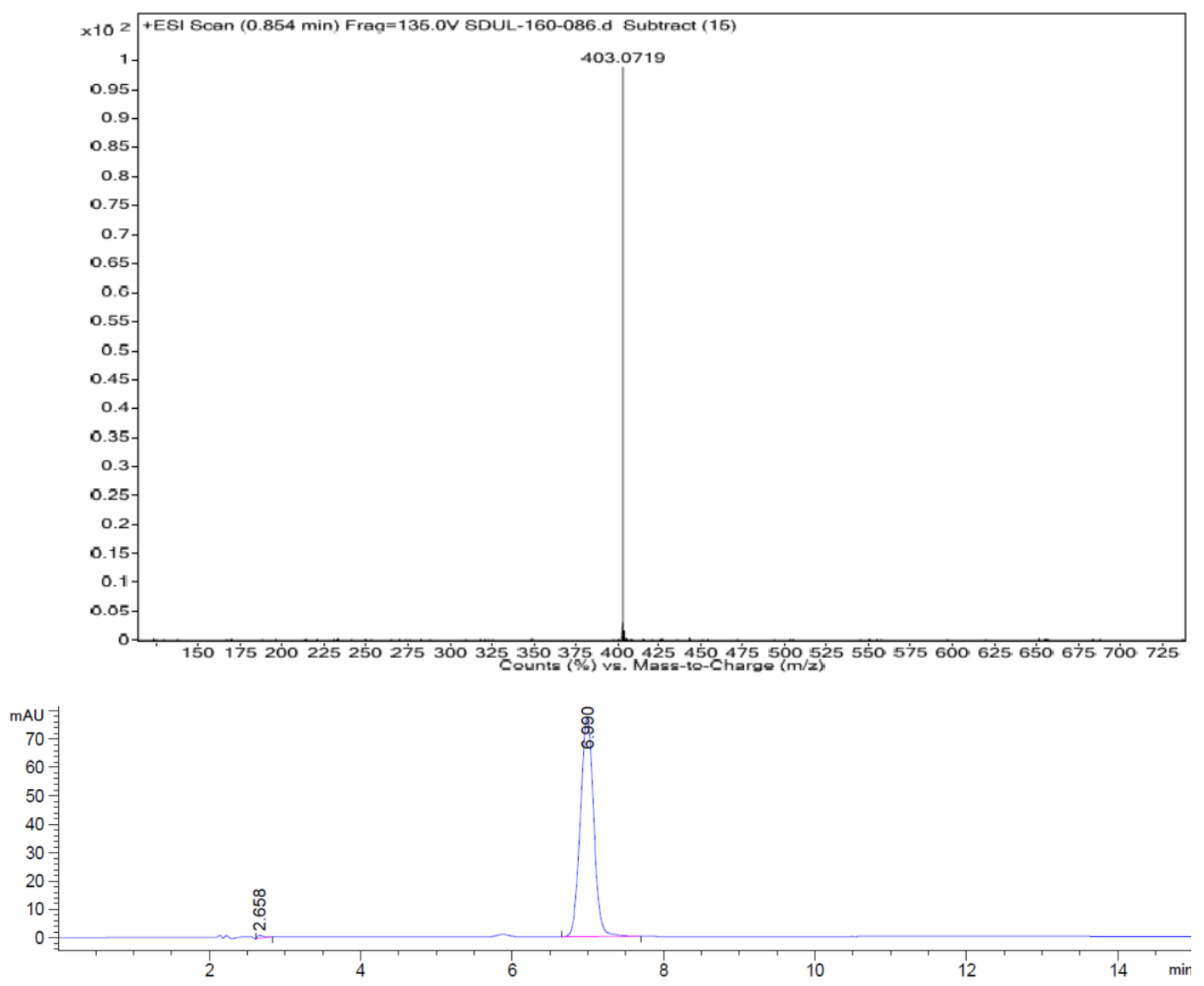

5-chloro-1,3-difluoro-2-nitrosobenzene (9-2) 

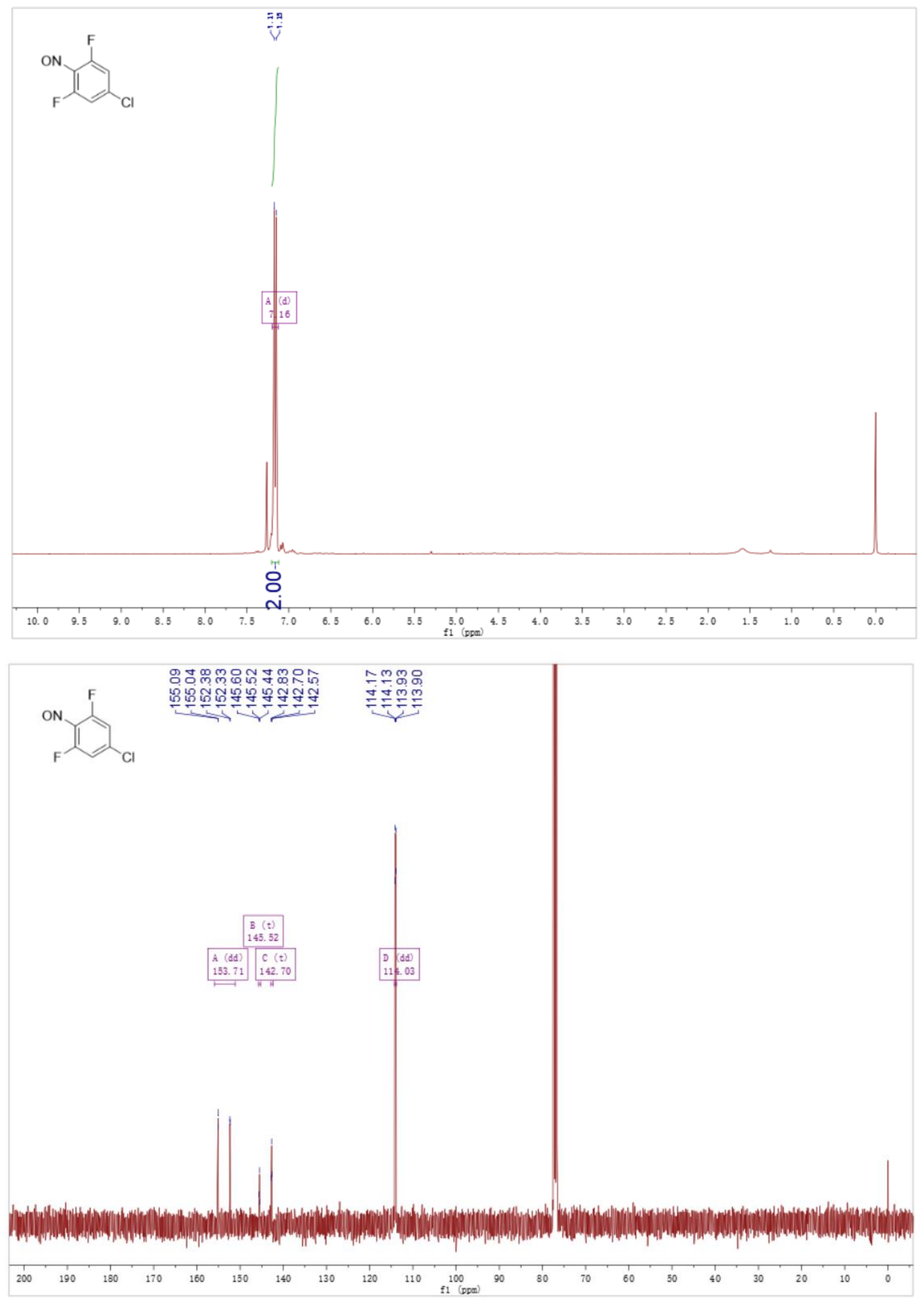


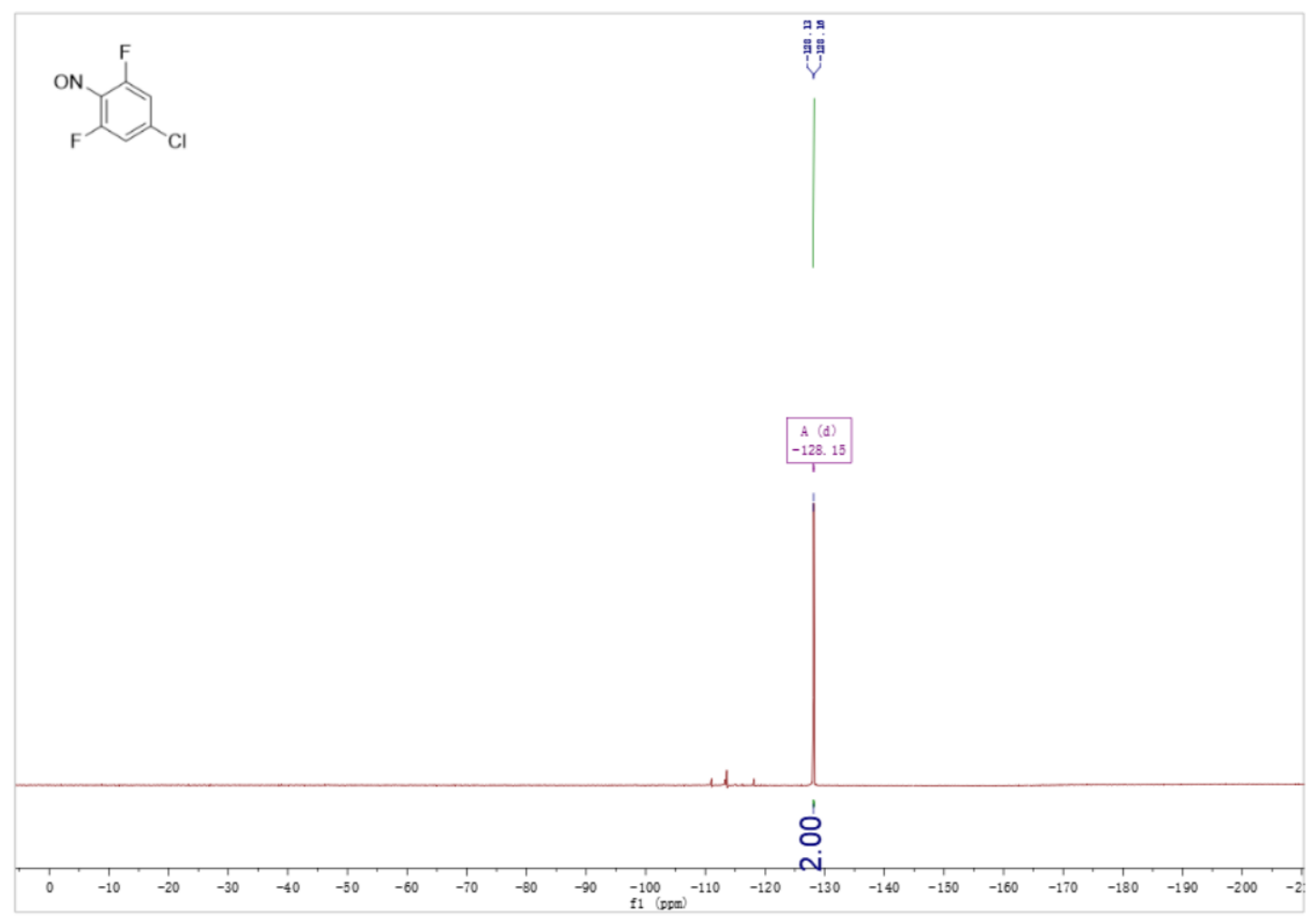

(E)-3-((4-chloro-2,6-difluorophenyl)diazenyl)-1H-pyrazole (9-3)

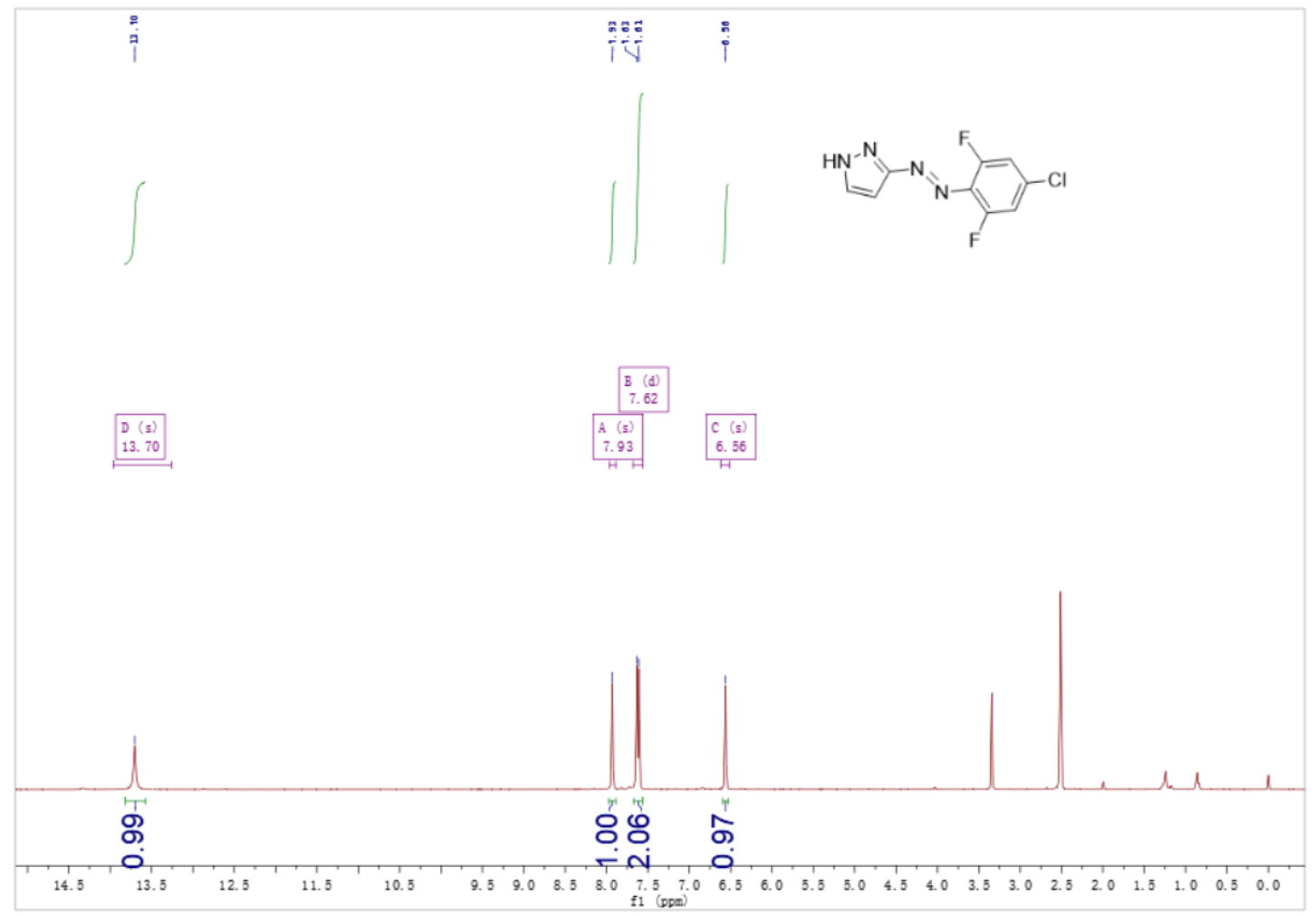



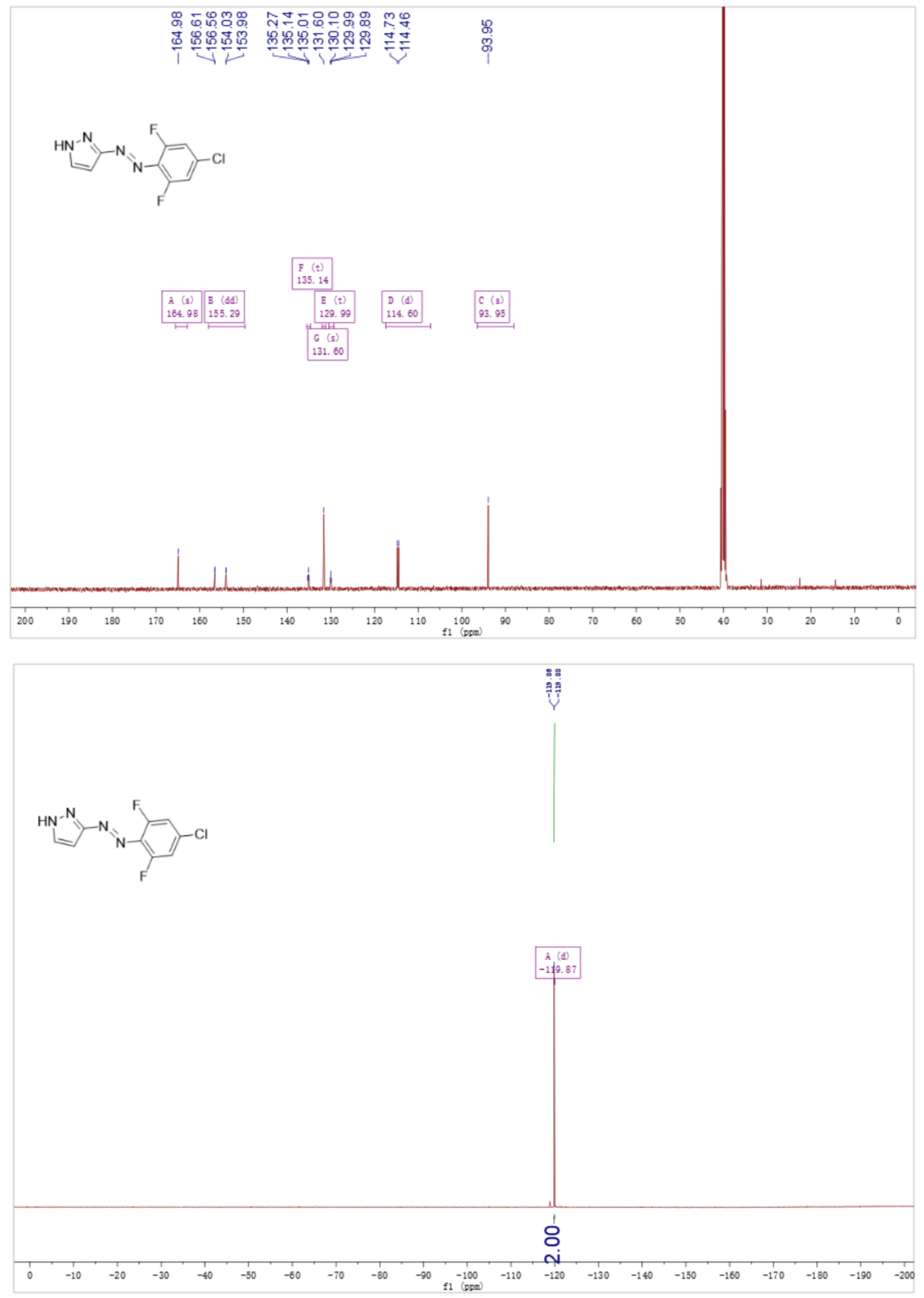


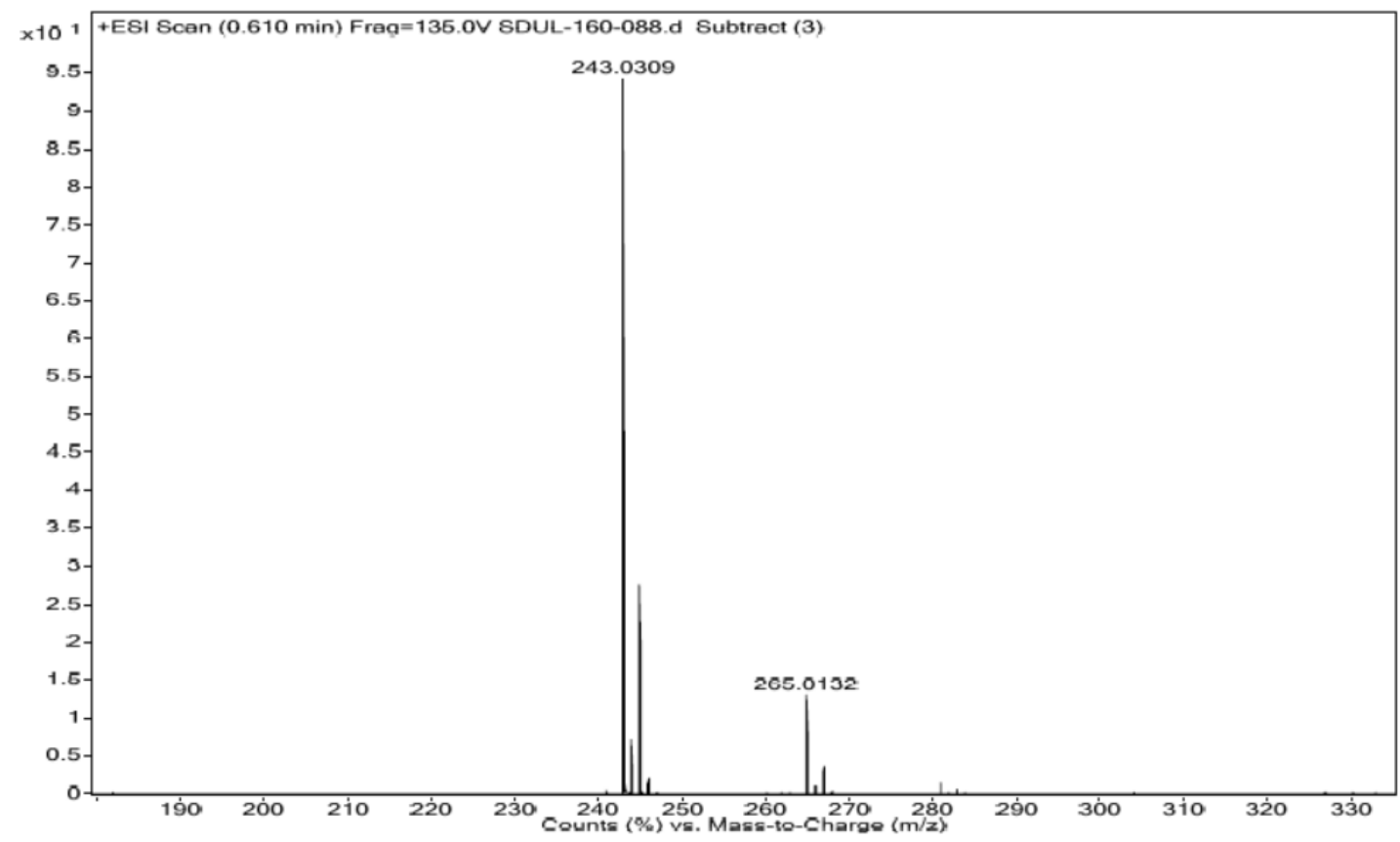

(E)-3-((4-chloro-2,6-difluorophenyl)diazenyl)-1-(2-fluoro-6-(trifluoromethyl)benzyl)-1H-pyrazole (piCRAC-9)

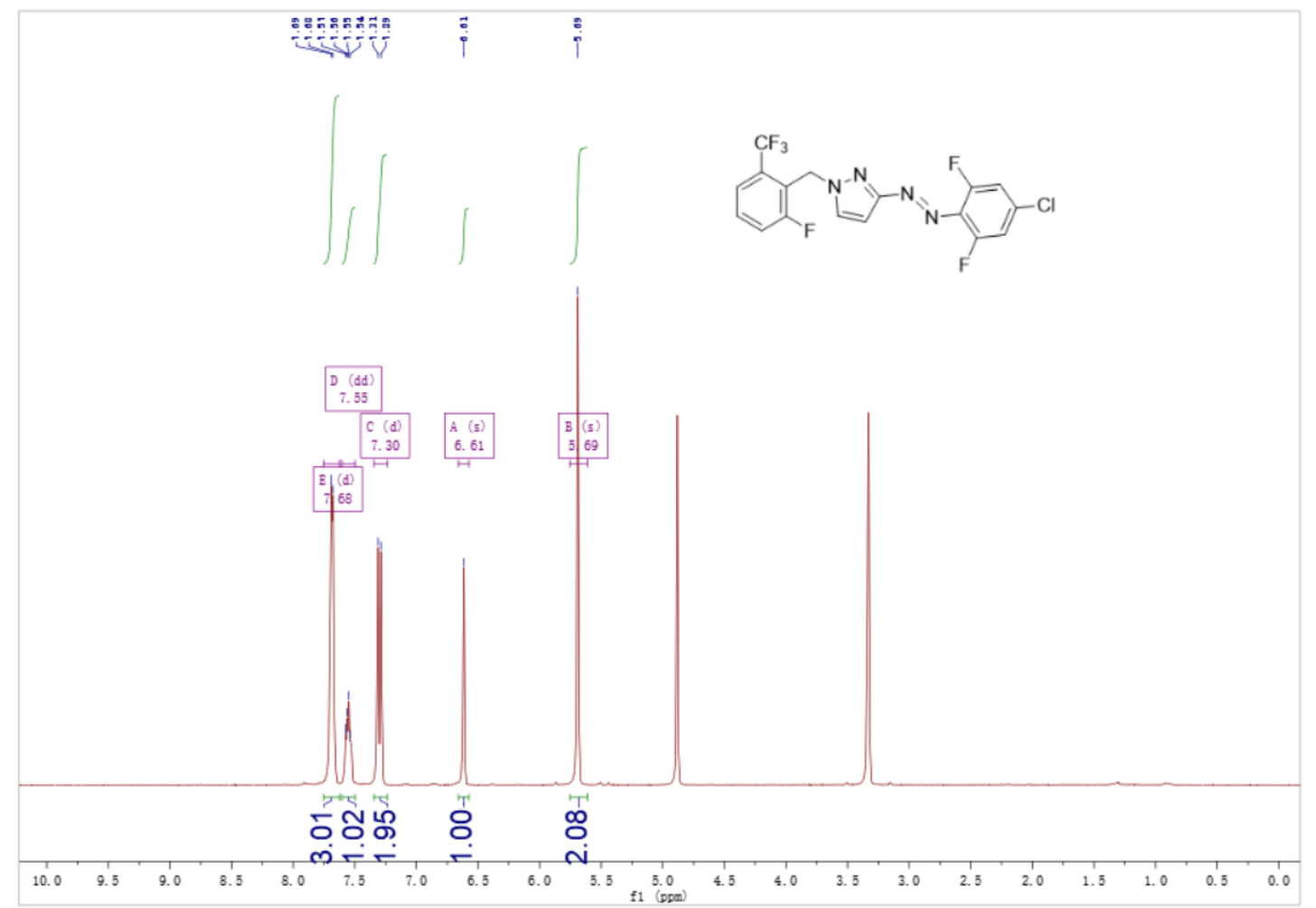



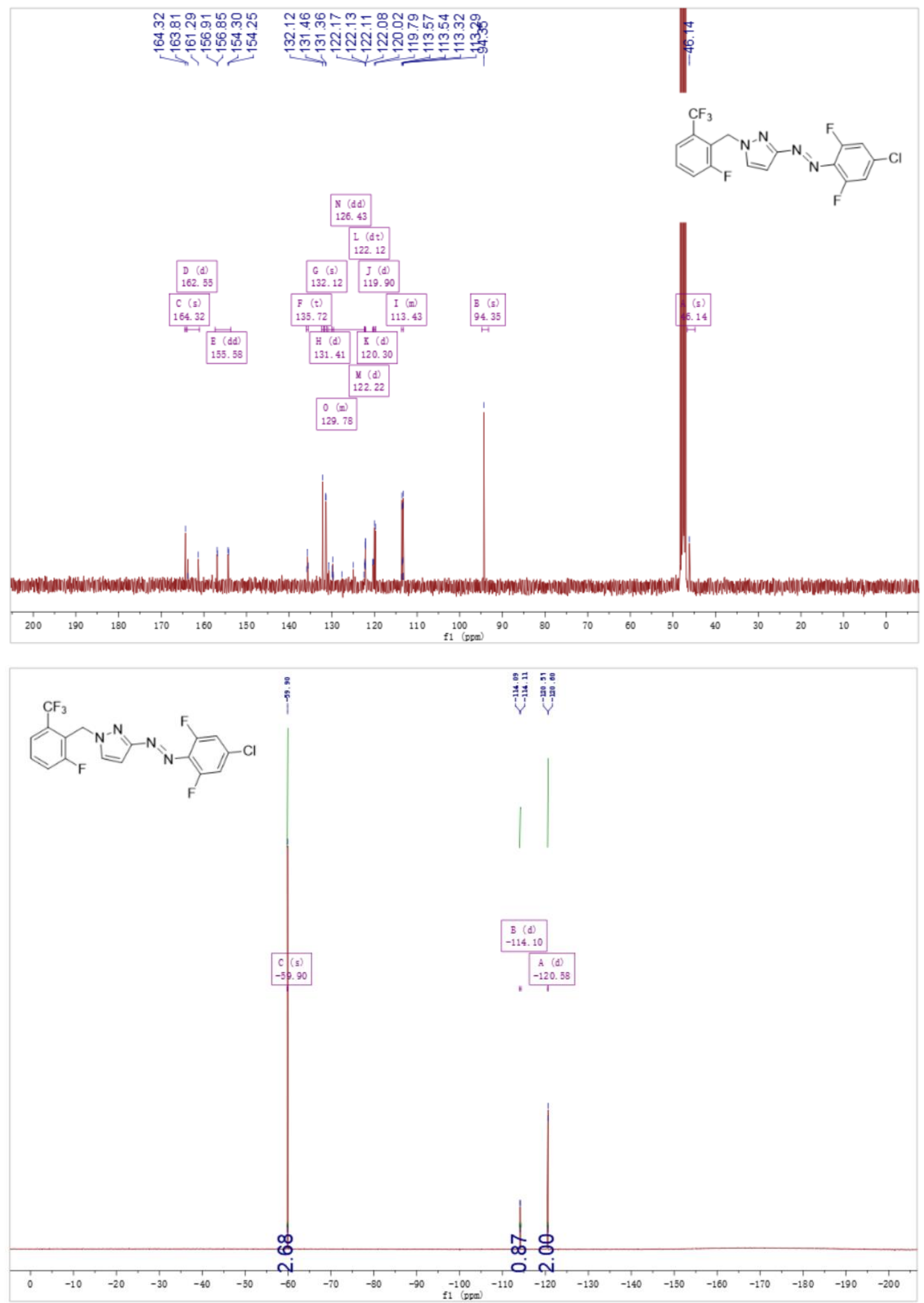

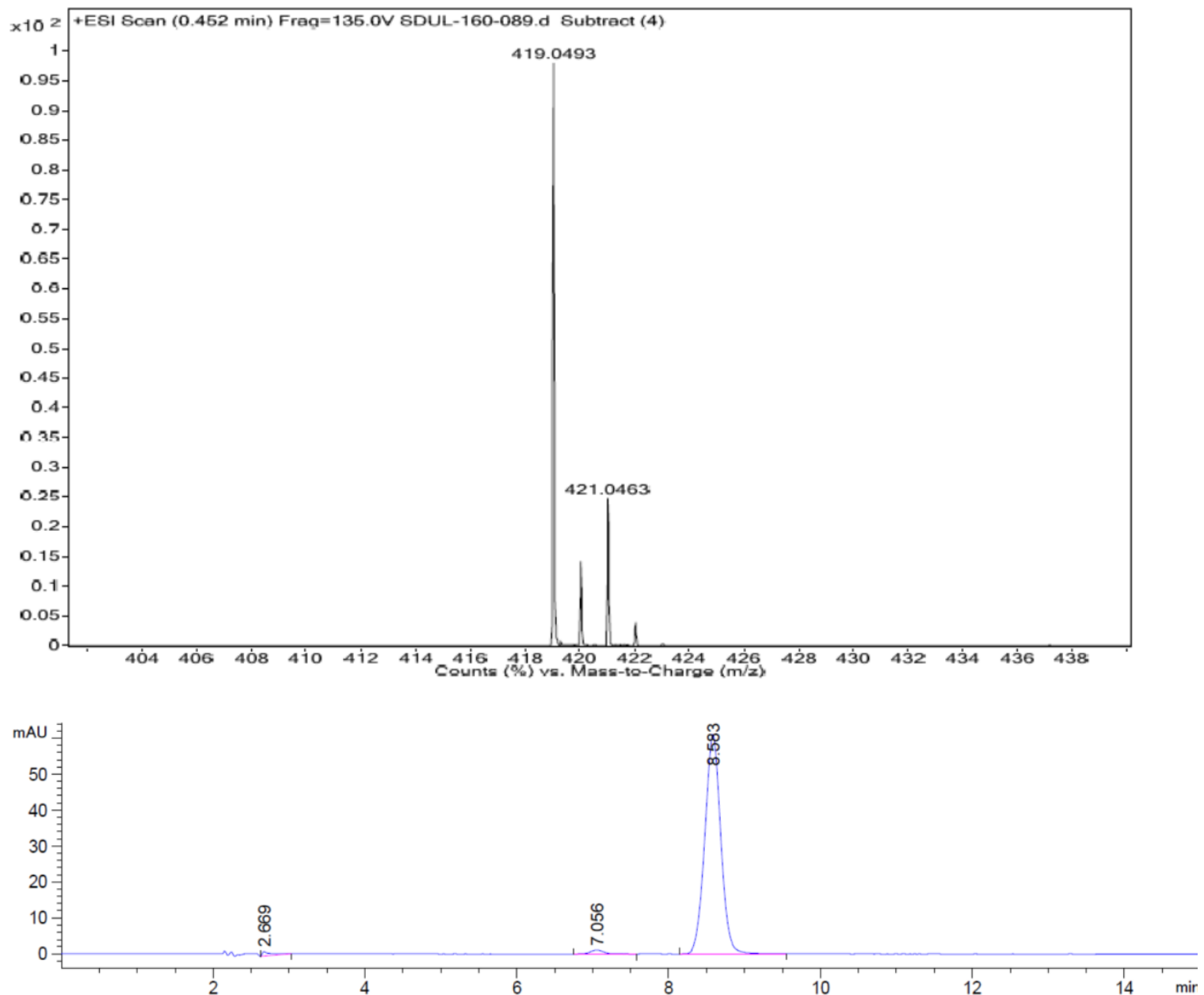

5-bromo-1,3-difluoro-2-nitrosobenzene (10-2) 

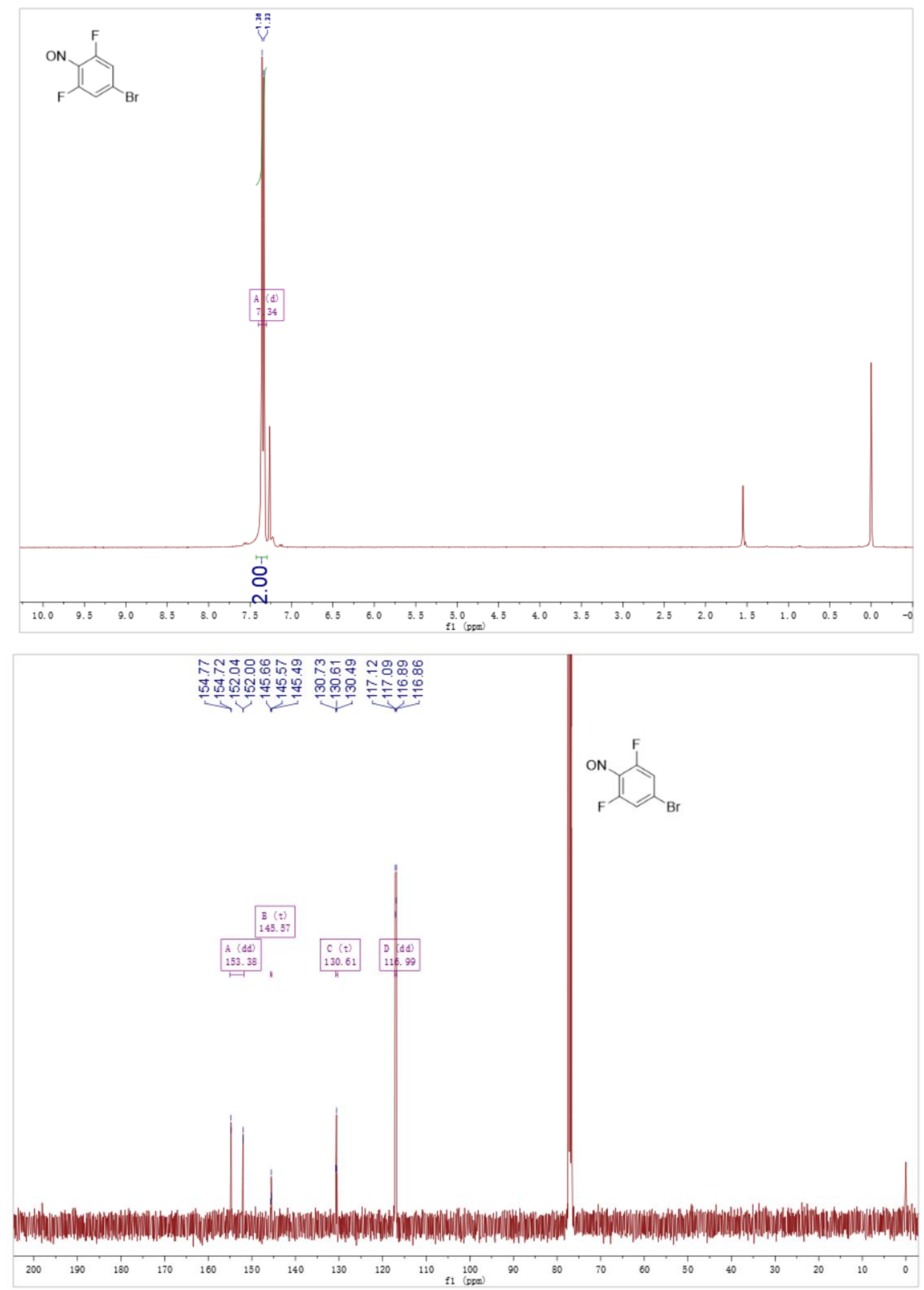


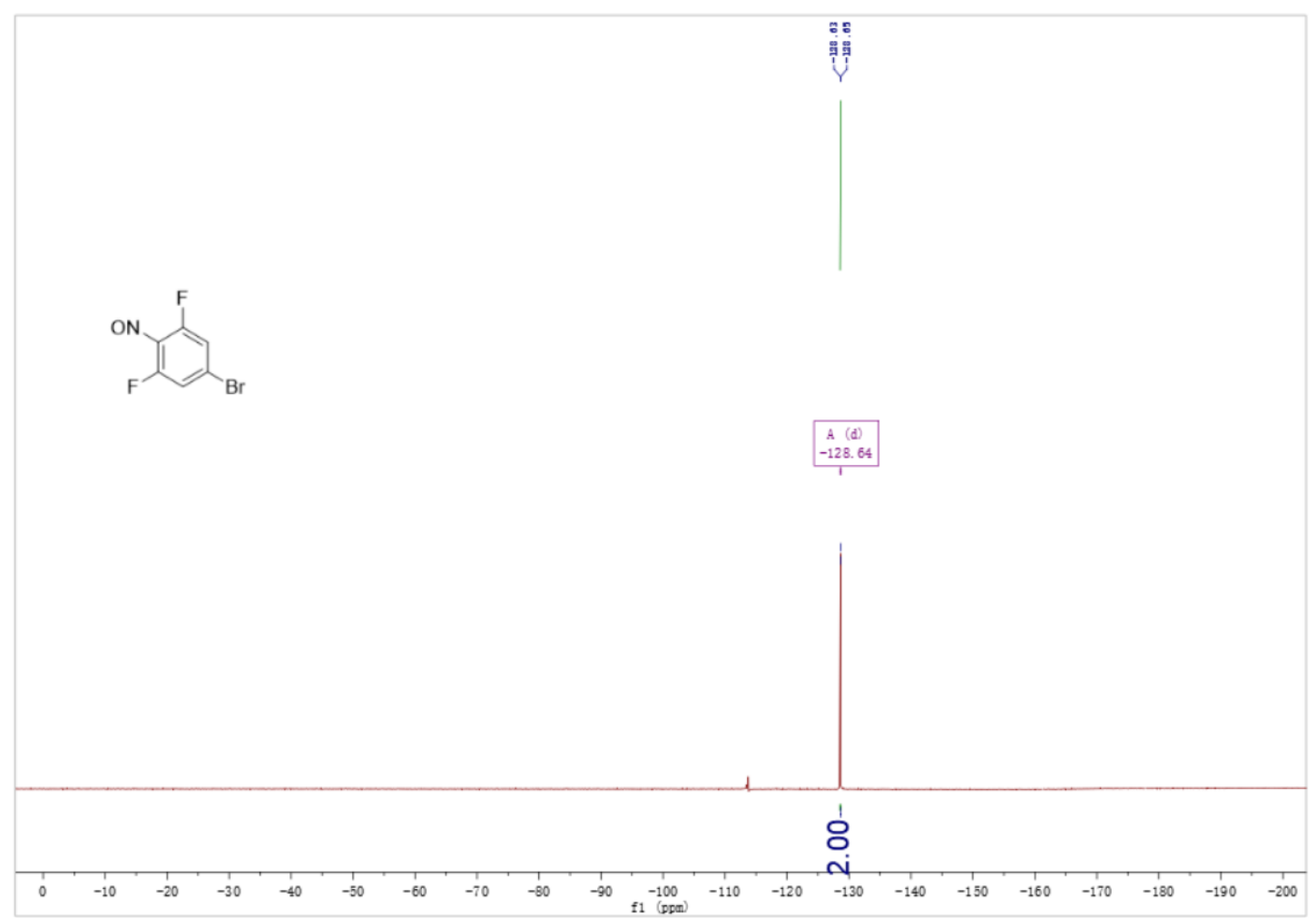

(E)-3-((4-bromo-2,6-difluorophenyl)diazenyl)-1H-pyrazole (10-3)

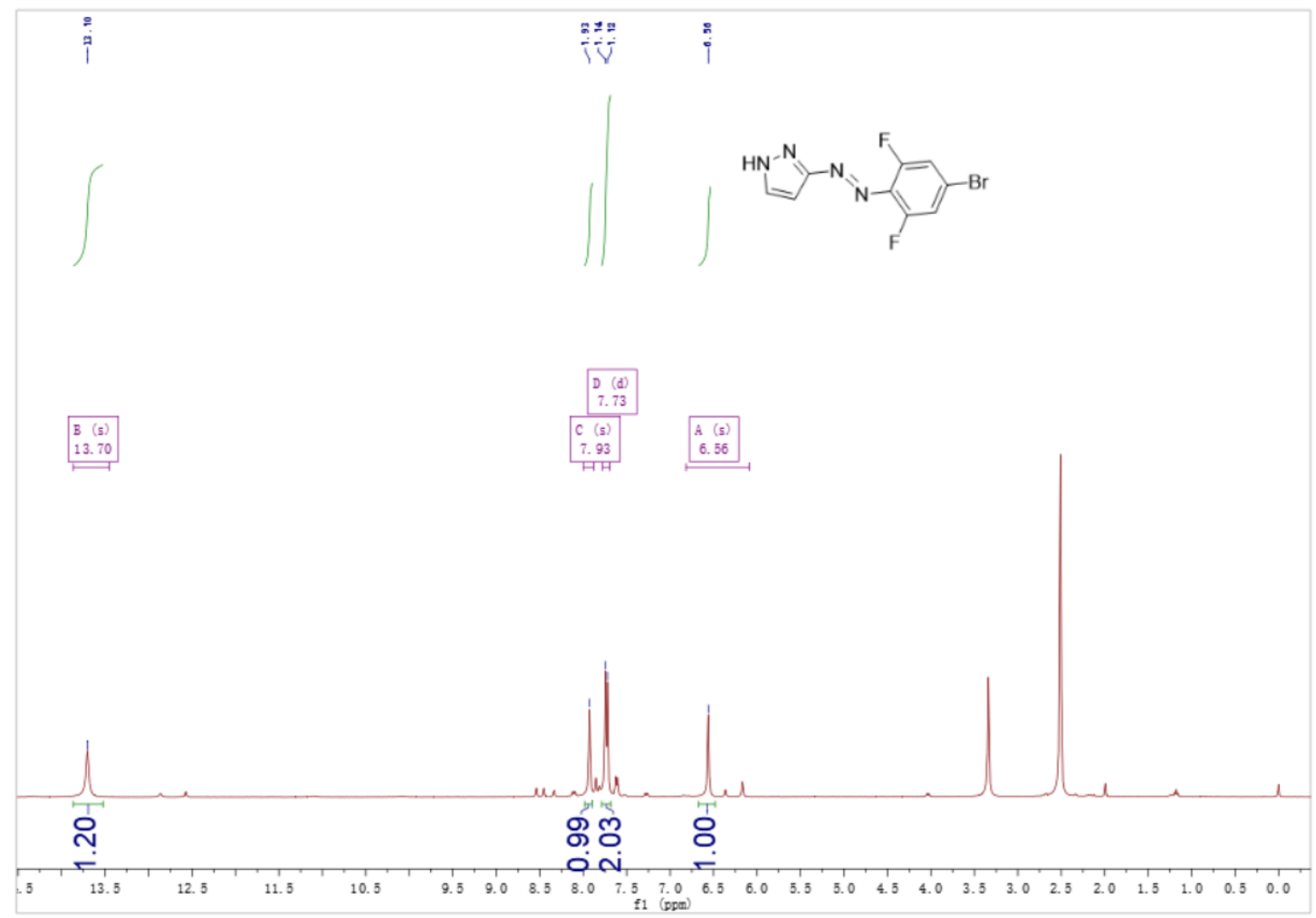



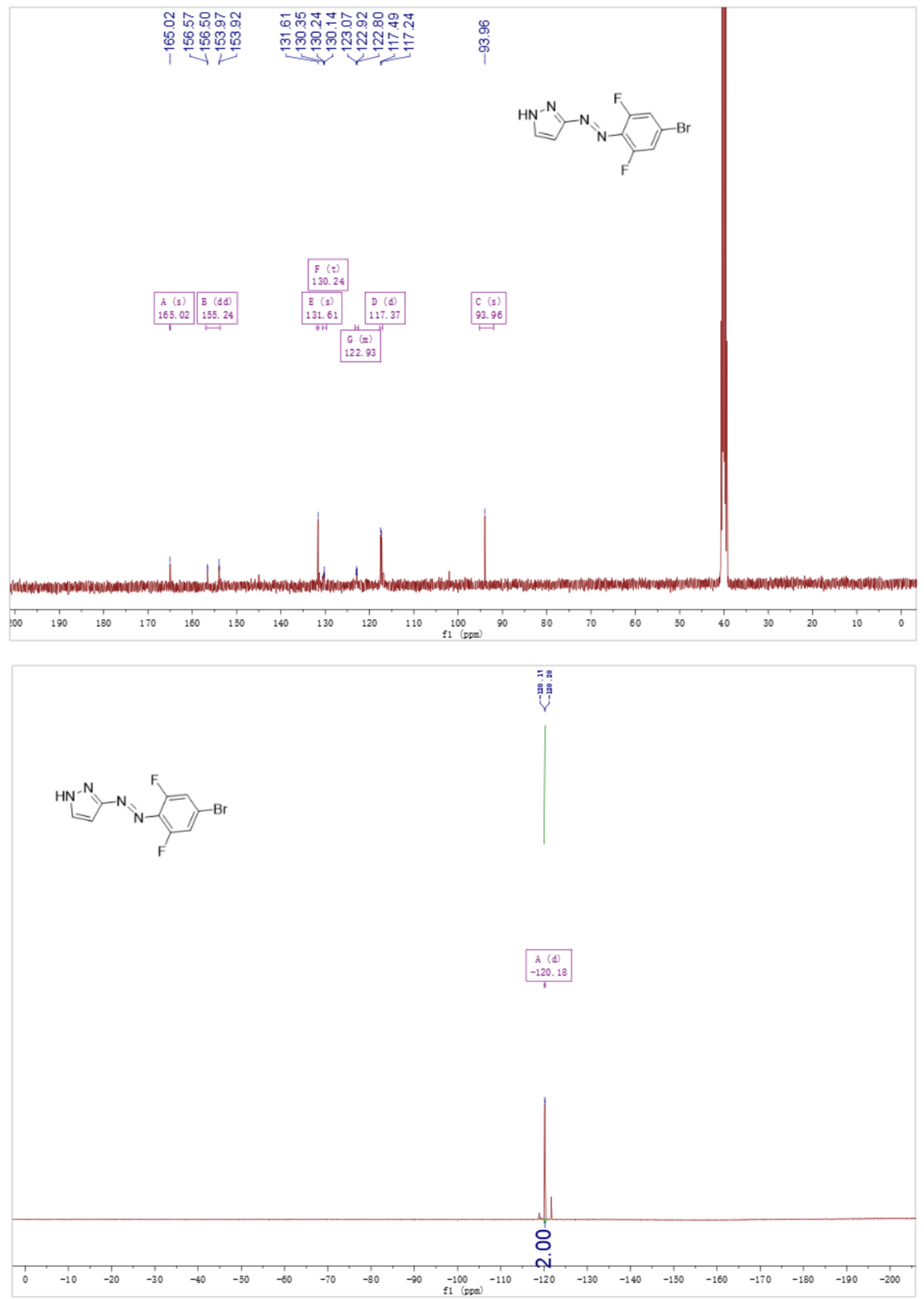

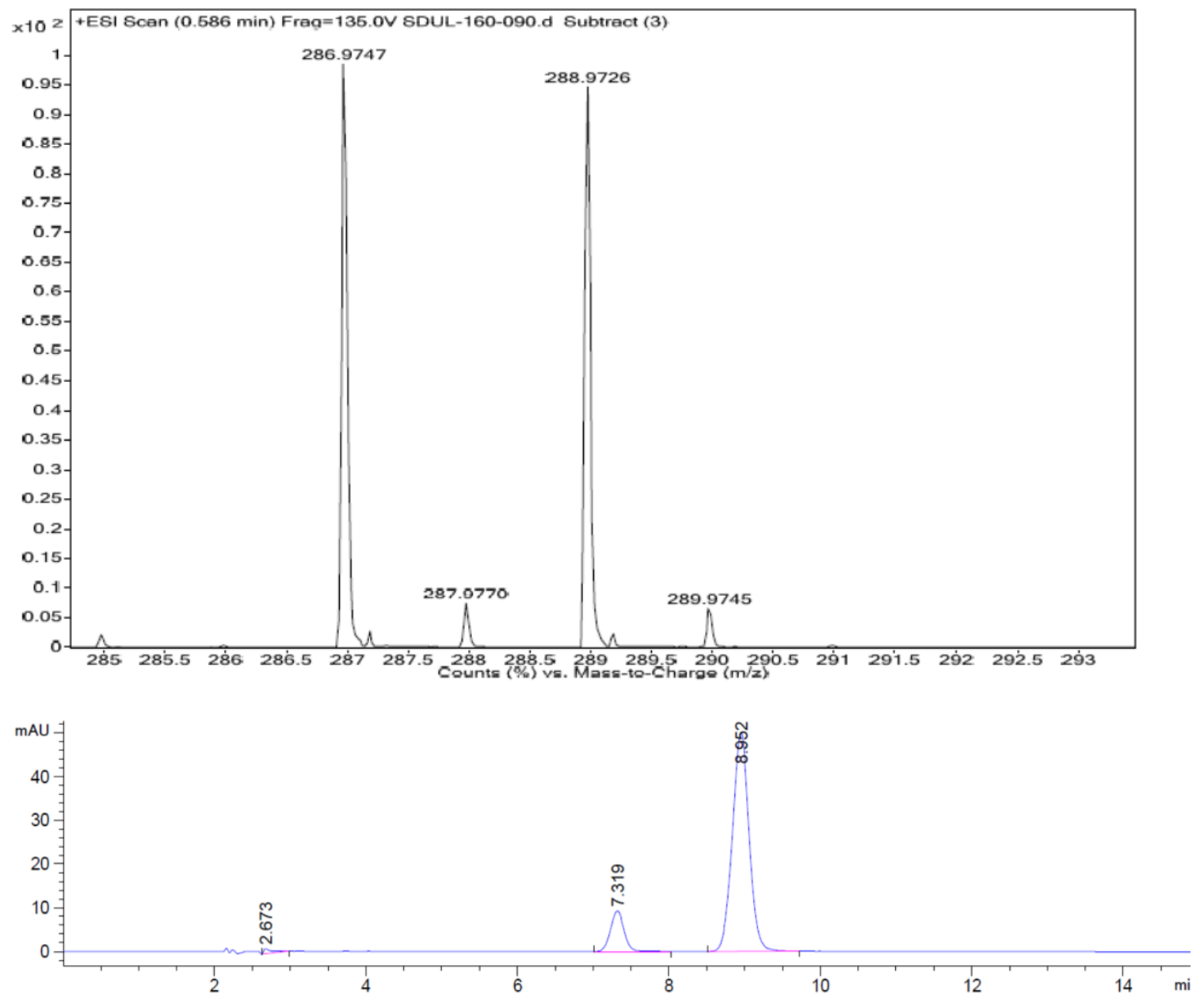